\title{
Standing wave instabilities in a chain of nonlinear coupled oscillators
}

\author{
Anna Maria Morgante ${ }^{\text {a }}$, Magnus Johansson ${ }^{\text {a,* }}$, \\ Georgios Kopidakis $^{\mathrm{a}, \mathrm{b}}$, Serge Aubry ${ }^{\mathrm{a}}$ \\ ${ }^{a}$ Laboratoire Léon Brillouin (CEA-CNRS), CEA Saclay, F-91191 Gif-sur-Yvette \\ Cedex, France \\ ${ }^{\mathrm{b}}$ Department of Physics, University of Crete, P.O. Box 2208, GR-71003 \\ Heraklion, Crete, Greece
}

\begin{abstract}
We consider existence and stability properties of nonlinear spatially periodic or quasiperiodic standing waves (SWs) in one-dimensional lattices of coupled anharmonic oscillators. Specifically, we consider Klein-Gordon (KG) chains with either soft (e.g., Morse) or hard (e.g., quartic) on-site potentials, as well as discrete nonlinear Schrödinger (DNLS) chains approximating the small-amplitude dynamics of KG chains with weak inter-site coupling. The SWs are constructed as exact timeperiodic multibreather solutions from the anticontinuous limit of uncoupled oscillators. In the validity regime of the DNLS approximation these solutions can be continued into the linear phonon band, where they merge into standard harmonic SWs. For SWs with incommensurate wave vectors, this continuation is associated with an inverse transition by breaking of analyticity. When the DNLS approximation is not valid, the continuation may be interrupted by bifurcations associated with resonances with higher harmonics of the SW. Concerning the stability, we identify one class of SWs which are always linearly stable close to the anticontinuous limit. However, approaching the linear limit all SWs with nontrivial wave vectors become unstable through oscillatory instabilities, persisting for arbitrarily small amplitudes in infinite lattices. Investigating the dynamics resulting from these instabilities, we find two qualitatively different regimes for wave vectors smaller than or larger than $\pi / 2$, respectively. In one regime persisting breathers are found, while in the other regime the system rapidly thermalizes.
\end{abstract}

Key words: Anharmonic lattices, Nonlinear standing waves, Oscillatory instabilities, Breaking of analyticity

PACS: 63.20.Ry, 45.05.+x, 05.45.-a, 42.65.Sf 


\section{Introduction}

It is well-known that the dynamics of spatially periodic harmonic systems is non-ergodic, since it can be completely described as a linear combination (with time-independent coefficients) of travelling waves of the form $\cos (Q x-\omega(Q) t)$ with wave vector $Q$ and frequency $\omega(Q)$. When there are (even small) anharmonic terms present, most harmonic solutions with amplitude zero are very unlikely to be continuable to finite amplitude, because it is generally believed (despite the famous work of Fermi, Pasta and Ulam [1]) that anharmonic terms in general introduce some ergodicity in the system, which in the long term should lead to its thermalization.

It is nevertheless an interesting question to understand whether special harmonic solutions could persist as exact solutions when the system becomes anharmonic. Actually, it has been proven recently that small-amplitude periodic travelling waves (with the form $\cos (Q x-\omega t)$ in the harmonic limit) do exist for Hamiltonian chains of linearly coupled nonlinear oscillators (KleinGordon (KG) lattices) [2] as well as for chains of mass particles with nonlinear nearest-neighbour interactions (Fermi-Pasta-Ulam (FPU) lattices) [3]. However, for KG lattices the unique continuation of harmonic travelling waves is possible only in a regime of weak inter-site coupling (i.e., close enough to the so-called anticontinuous limit [4] of uncoupled oscillators); beyond this regime, the situation is more complex and there are many bifurcations where new solutions appear (such as nanopterons, which are travelling localized waves with a noncancelling periodic tail at infinity) $[2,5]$. In the simpler regime close to the anticontinuous limit, numerical techniques for finding time-periodic propagating waves, based on the concept of discrete breathers (see e.g. [4,6] for reviews of recent developments on discrete breathers), were implemented in [7]. The solutions were constructed as multi-site breathers ('multibreathers') from the trivial solution at the anticontinuous limit where all oscillators are oscillating in phase with the same frequency. An arbitrary spatially uniform phase torsion was then applied, and a Newton scheme used to continue these solutions to non-zero coupling according to the theory described in [4].

However, as was found already in [8] from a discrete nonlinear Schrödinger (DNLS) approximation (generally valid for small-amplitude oscillations and small coupling, see also Sec. 2.2 below), propagating waves in KG lattices may exhibit a modulational (Benjamin-Feir) instability by which an initially uniform wave breaks up into an array of localized pulses. Depending on whether

\footnotetext{
* Corresponding author. Present address: Department of Physics and Measurement Technology, Linköping University, S-581 83 Linköping, Sweden.

Email addresses: morgante@llb.saclay.cea.fr (Anna Maria Morgante), mjn@ifm.liu.se (Magnus Johansson), kopidaki@llb.saclay.cea.fr (Georgios Kopidakis), aubry@llb.saclay.cea.fr (Serge Aubry).
} 
the $\mathrm{KG}$ on-site potential is soft or hard (i.e. whether the oscillation frequency decreases or increases with increasing oscillation amplitude for the individual oscillators), the modulational instability occurs for either small or large wave vectors of the travelling wave. (A similar result has been found also for FPU lattices [9].) This instability also appears a consequence of the theory of effective action for multibreathers with phase torsion as developed in [4] (see also further developments in [10]).

In this paper, we are interested in the continuation of standing waves ( $S W s$ ), which in the harmonic limit are superpositions of two travelling waves with the same amplitude and opposite wave vectors, and thus have the form $\cos (Q x) \cos (\omega t)$. We shall here study such solutions for discrete KG systems, and for the related DNLS system describing small-amplitude solutions close to the anticontinuous limit. Our approach for finding these solutions is similar to that used in [11] for disordered KG lattices: we construct appropriate multibreather solutions with frequency outside the linear phonon band at the anticontinuous limit and continue them to nonzero coupling; these solutions are then continued versus frequency inside the phonon band. We shall propose an ansatz for finding the appropriate coding sequence $[12,4]$ for these multibreathers, allowing for a smooth continuation of the multibreather versus frequency down to zero amplitude as a linear SW. When the spatial period of the SW is incommensurate with the lattice period, its envelope ( $h u l l$ ) function undergoes a 'transition by breaking of analyticity' (TBA) [13-15] at some critical value of its frequency.

Investigating the linear stability of these nonlinear SWs in infinite lattices, we find the rather striking result that although there exist linearly stable SWs close to the anticontinuous limit, all SWs with nontrivial wave vectors (i.e. different from 0 or $\pi$ ) become linearly unstable through an oscillatory instability bifurcation when approaching the linear limit. Thus, small-amplitude nonlinear SWs will be unstable also for wave numbers where the corresponding propagating waves are modulationally stable. These extended instabilities are a direct consequence of discreteness, and have not been found within earlier continuum approximations [16]. However, similarly as found e.g. in [17,18], the instabilities are very sensitive to finite size effects, so that in a finite system linear stability will generally be recovered close to the linear limit.

Moreover, we show that when their hull function is non-analytic, these SWs can be viewed as arrays of discommensurations (see, e.g., [19]) of a simple stationary phonon with wave vector $\pi$ or 0 , and that each discommensuration is unstable (as was found already in [18] for the DNLS model). The effect of the instability on a single discommensuration is analysed with the technique developed in $[20,21]$, and it is found that, after an initial oscillatory regime, it moves spontaneously in the lattice with a rather well-defined velocity, analogously to the behaviour of the discrete dark mode in the DNLS model found in 
$[22,18]$. The long-time behaviour of the unstable SWs can then be interpreted as the result of inelastic interactions between individual moving discommensurations. As for the DNLS model the SW with wave vector $\pi / 2$ is found to exactly coincide with the line of phase transition recently discovered [23] in this model, SWs with wave vectors larger or smaller than $\pi / 2$ belong to different phases and therefore yield qualitatively different asymptotic dynamics: one phase corresponds to normal thermalization, while in the other phase persisting large-amplitude localized excitations occur. As we will find from numerical simulations, a similar scenario also occurs for KG chains with small inter-site coupling.

The layout of this paper is as follows. In Section 2, we describe the KG model and derive a DNLS-type equation for its small-amplitude dynamics. This equation generally contains long-range nonlinear interactions, but reduces to the ordinary nearest-neighbour DNLS equation in the limit of small coupling. In Section 3, we describe the representation of nonlinear SWs as trajectories of a symplectic map, which in the DNLS approximation reduces to a cubic map in the 2-dimensional real plane [24]. The breakup of Kol'mogorov-Arnol'dMoser (KAM) tori for this map corresponds to a TBA for the hull function of incommensurate SWs, and the continuation of periodic and quasiperiodic map trajectories to the anticontinuous limit yields the SW multibreather coding sequences. In Section 4, we study numerically and analytically the linear stability properties of SWs within the DNLS approximation, where in particular the existence of oscillatory instabilities close to the linear limit can be proven using perturbation theory. In Section 5, SWs in the full KG model are analyzed numerically for two particular examples of soft (Morse) and hard (quartic) on-site potentials. Their linear stability properties are investigated using numerical Floquet analysis, and bifurcations determining the limit of existence for larger coupling are identified. In Section 6, we discuss the dynamics resulting from the SW instabilities over short, intermediate, and long time scales, and Section 7 concludes the paper. The more technical parts of the stability analysis for the DNLS case is deferred to the Appendix. Some of the results obtained in this paper have been presented in an abbreviated form elsewhere [25].

\section{The model}




\subsection{The Klein-Gordon system}

We consider a one-dimensional chain of classical anharmonic oscillators (KleinGordon chain) (Fig. 1a) with Hamiltonian

$$
H=\sum_{n=1}^{N}\left[\frac{1}{2} \dot{u}_{n}^{2}+V\left(u_{n}\right)+\frac{1}{2} C_{K}\left(u_{n+1}-u_{n}\right)^{2}\right] \equiv \sum_{n=1}^{N} H_{n}
$$

where the total energy attributed to each site $n$ is defined as

$$
H_{n} \equiv \frac{1}{2} \dot{u}_{n}^{2}+V\left(u_{n}\right)+\frac{1}{4} C_{K}\left(\left(u_{n+1}-u_{n}\right)^{2}+\left(u_{n-1}-u_{n}\right)^{2}\right) .
$$

The oscillators are submitted to a nonlinear on-site potential $V(u)$ and are coupled by a harmonic inter-site coupling $C_{K}>0$. The nonlinear potential can be either a soft potential such as the Morse potential (Fig. 1b)

$$
V(u)=\frac{1}{2} \omega_{0}^{2}\left(\mathrm{e}^{-u}-1\right)^{2}
$$

or a hard potential such as the quartic potential (Fig. 1c)

$$
V(u)=\omega_{0}^{2}\left(\frac{u^{2}}{2}+\frac{u^{4}}{4}\right)
$$

(Recall that for a soft [hard] potential the oscillation frequency decreases [increases] with the oscillation amplitude or, equivalently, with the action.) In the rest of the paper we will consider $V^{\prime \prime}(0)=\omega_{0}^{2}=1$.

The choice of the Morse potential is physically justified by the fact that it represents an appropriate description of hydrogen bonds, and it is then especially useful for the study of biological systems such as macromolecules and proteins.

The equations of motion for the particle displacements $u_{n}$ then take the form of a discrete nonlinear $\mathrm{KG}$ equation,

$$
\ddot{u}_{n}+V^{\prime}\left(u_{n}\right)-C_{K}\left(u_{n+1}+u_{n-1}-2 u_{n}\right)=0 \text {. }
$$

For a given solution $u_{n}(t)$, the corresponding linearized equations (Hill equations) are

$$
\ddot{\epsilon}_{n}+\epsilon_{n} V^{\prime \prime}\left(u_{n}\right)-C_{K}\left(\epsilon_{n+1}+\epsilon_{n-1}-2 \epsilon_{n}\right)=0
$$



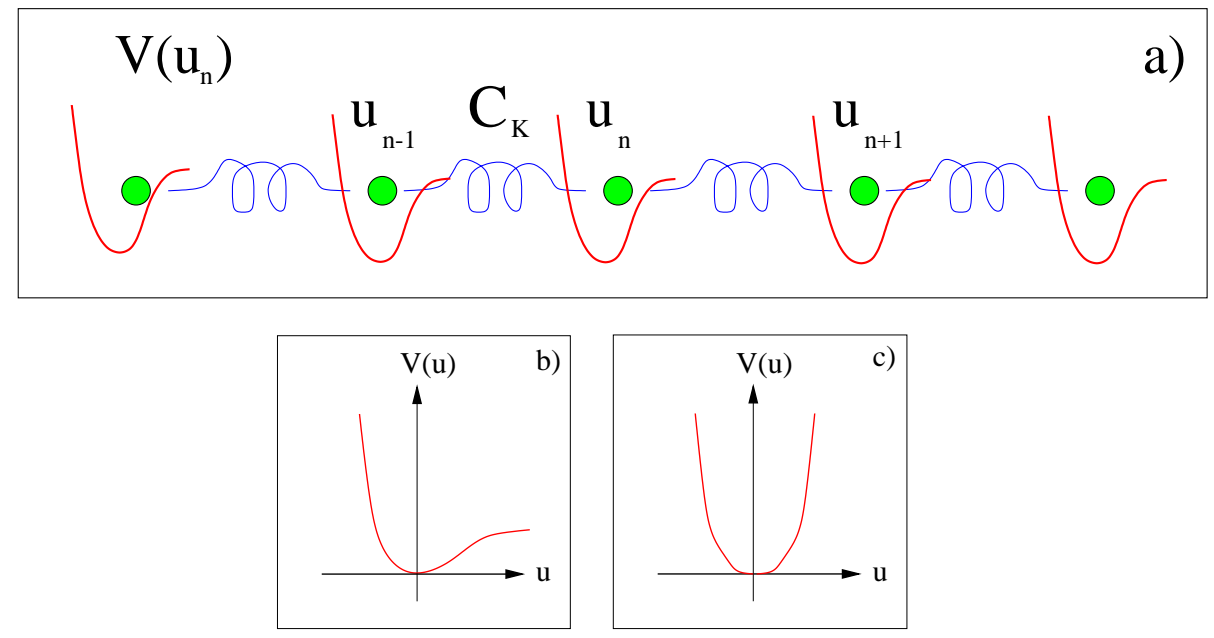

Fig. 1. (a) Klein-Gordon chain of nonlinear oscillators with displacement $\{u\}$, coupled by a constant force $C_{K}$; (b) the soft Morse potential; (c) the hard quartic potential.

where $\epsilon_{n}$ denotes a small displacement with respect to the solution $u_{n}$. When $u_{n}=0$ (or, more generally, when $V^{\prime \prime}\left(u_{n}\right)$ is constant), the standard harmonic (plane-wave) small-amplitude solutions

$$
\epsilon_{n}(t)=\epsilon \cos \left(Q n-\omega_{l}(Q) t\right)
$$

with wave vector $Q$ and frequency $\omega_{l}(Q)$ are obtained. By substitution of these solutions in Eq. (6), we find the (phonon) dispersion relation

$$
\omega_{l}^{2}(Q)=1+4 C_{K} \sin ^{2} Q / 2
$$

for small-amplitude oscillations in a general potential.

\subsection{Small amplitude limit and DNLS equation}

We will now show how it is possible to describe the small-amplitude dynamics of general KG chains with small inter-site coupling $C_{K}$ by a DNLS approximation. Although similar results have been obtained earlier $[8,16]$, our approach is slightly different and offers a deeper understanding for the role of the dif-

ferent limits involved. Considering small-amplitude solutions to the nonlinear discrete KG system (5), the anharmonic terms will be small, and the on-site anharmonic potential $V(u)$ can be expanded at the lowest significant order as

$$
V(u)=\frac{1}{2} u^{2}+\alpha \frac{u^{3}}{3}+\beta \frac{u^{4}}{4}+\ldots
$$


For example, in the case of the Morse potential, the expansion yields $\alpha=-3 / 2$ and $\beta=7 / 6$, while in the case of the quartic potential $\alpha=0$ and $\beta=1$.

We then search for solutions of the nonlinear equation (5) which are locally close to linearized phonons (7) with wave vector $Q$ and small amplitude $\epsilon$. If such a solution $u_{n}(t)$ is assumed to be time periodic with period $T_{b}=\frac{2 \pi}{\omega_{b}}$, where $\omega_{b}$ is close to some phonon frequency $\omega_{l}(Q)$, it can be expanded as a Fourier series,

$$
u_{n}(t)=\sum_{p} a_{n}^{(p)} \mathrm{e}^{\mathrm{i} p \omega_{b} t}
$$

where the complex coefficients fulfill $a_{n}^{(p)}=a_{n}^{(-p) *}$. For a time-reversible solution, the coefficients $a_{n}^{(p)}$ will be real. Moreover, we require the Fourier series to be summable as a smooth (say analytic) function of $t$ and thus that the coefficients $a_{n}^{(p)}$ must decay asymptotically exponentially with $p$ for each $n$.

More generally, since for the linear solution the left-hand side of Eq. (5) is not strictly vanishing but of order $\epsilon^{2}$, the perturbed solution will have a harmonic time dependence plus corrections of order $\epsilon^{2}$. Thus, as in Refs. $[8,16]$, we search for general (not necessarily time-periodic) small-amplitude solutions as series with the form (10) but where the Fourier coefficients depend slowly on time as a function of $\epsilon^{2} t$. Then, in the Fourier series (10), $a_{n}^{(1) *}=a_{n}^{(-1)}$ are the leading order terms of order $\epsilon, a_{n}^{(0)}$ and $a_{n}^{(2) *}=a_{n}^{(-2)}$ are smaller at order $\epsilon^{2}$, and, since the Fourier components must decay exponentially, it is natural to assume that $a_{n}^{(3) *}=a_{n}^{(-3)}$ are of order $\epsilon^{3}, a_{n}^{(4) *}=a_{n}^{(-4)}$ are of order $\epsilon^{4}$, etc. Then solving Eq. (5) at order $\epsilon^{3}$ yields ${ }^{1}$, with $\omega_{b}^{2}-1 \equiv 2 \delta$, for each harmonic

$$
\begin{aligned}
a_{n}^{(0)}+2 \alpha\left|a_{n}^{(1)}\right|^{2}-C_{K}\left(a_{n+1}^{(0)}+a_{n-1}^{(0)}-2 a_{n}^{(0)}\right) & =0 \\
2 i \omega_{b} \dot{a}_{n}^{(1)}-2 \delta a_{n}^{(1)}+2 \alpha\left(a_{n}^{(1)} a_{n}^{(0)}+a_{n}^{(1) *} a_{n}^{(2)}\right)+3 \beta\left|a_{n}^{(1)}\right|^{2} a_{n}^{(1)} & \\
-C_{K}\left(a_{n+1}^{(1)}+a_{n-1}^{(1)}-2 a_{n}^{(1)}\right) & =0 \\
\left(1-4 \omega_{b}^{2}\right) a_{n}^{(2)}+\alpha a_{n}^{(1)}{ }^{2}-C_{K}\left(a_{n+1}^{(2)}+a_{n-1}^{(2)}-2 a_{n}^{(2)}\right) & =0 \\
\left(1-9 \omega_{b}^{2}\right) a_{n}^{(3)}+2 \alpha a_{n}^{(1)} a_{n}^{(2)}+\beta a_{n}^{(1)}{ }^{3}-C_{K}\left(a_{n+1}^{(3)}+a_{n-1}^{(3)}-2 a_{n}^{(3)}\right) & =0 .
\end{aligned}
$$

The term $a_{n}^{(0)}$ can be obtained explicitly from Eq. (11) which yields

$$
a_{n}^{(0)}=A \sum_{m} \eta^{|n-m|}\left|a_{m}^{(1)}\right|^{2} \quad \text { with }
$$

$\overline{1 \text { Order }} \epsilon^{3}$ is the lowest order taking into account the anharmonicity of the local potential $V(u)$. 


$$
\eta=1-\frac{1}{2 C_{K}}\left(\sqrt{1+4 C_{K}}-1\right) \quad \text { and } \quad A=-\frac{2 \alpha}{\sqrt{1+4 C_{K}}}
$$

Similarly, $a_{n}^{(2)}$ can be obtained explicitly from Eq. (13) but only when $2 \omega_{b}>$ $\sqrt{1+4 C_{K}}=\omega_{l}(\pi)$, which means that the second harmonic of the solution does not belong to the linear phonon band:

$$
\begin{aligned}
a_{n}^{(2)} & =A^{\prime} \sum_{m} \eta^{\prime|n-m|} a_{m}^{(1) 2} \text { with } \\
\eta^{\prime} & =-1+\frac{4 \omega_{b}^{2}-1-4 C_{K}}{2 C_{K}}\left(\sqrt{1+\frac{4 C_{K}}{4 \omega_{b}^{2}-1-4 C_{K}}}-1\right) \text { and } \\
A^{\prime} & =\frac{\alpha}{\sqrt{\left(4 \omega_{b}^{2}-1-4 C_{K}\right)\left(4 \omega_{b}^{2}-1\right)}} .
\end{aligned}
$$

The substitution of Eqs. (15) and (17) in Eq. (12) yields a time-dependent extended DNLS equation with long range nonlinear interactions describing the slow time evolution of the dominating Fourier coefficients $a_{n}^{(1)}$, where the nonlinearities are taken into account to leading order in the amplitude $\epsilon$. If the condition $2 \omega_{b}>\sqrt{1+4 C_{K}}$ is not fulfilled, $\left|\eta^{\prime}\right|=1\left(\eta^{\prime}\right.$ complex, on the unit circle) and the sum (17) defining the interaction range in general diverges. Thus, for a general potential with non-zero $\alpha$, this condition imposes an upper validity limit for the coupling strength $C_{K}$ for this approach. (In the linear limit $\omega_{b}=\omega_{l}(Q)$, the condition is fulfilled for all $Q$ only when $C_{K}<3 / 4$.)

In the particular case when $\alpha=0$ (e.g. the quartic potential), there are no long-range terms in Eq. (12) [2 which immediately reduces to the ordinary DNLS equation with only nearest-neighbour interactions (Eq. (19) below). For a general potential, we consider the case of small $C_{K}$ (i.e. close to the anticontinuous limit $\left.C_{K}=0\right)$. Then, $\omega_{b}^{2}-1=2 \delta$ will be small of order of $C_{K}$, and $\eta=C_{K}+\mathcal{O}\left(C_{K}^{2}\right), \eta^{\prime}=-\frac{C_{K}}{4 \omega_{b}^{2}-1}+\mathcal{O}\left(C_{K}^{2}\right)$. Considering the modified DNLS equation (12) at order $C_{K}$ and neglecting terms of order $\epsilon^{3} C_{K}$, the long-range terms disappear and it reduces to the standard DNLS equation which well describes the dynamics of small-amplitude oscillations in KG chains close to the anti-continuous limit:

$$
i \dot{\psi}_{n}=\delta \psi_{n}-\sigma\left|\psi_{n}\right|^{2} \psi_{n}+C\left(\psi_{n+1}+\psi_{n-1}-2 \psi_{n}\right)
$$

where $\psi_{n}=\sqrt{|\lambda|} a_{n}^{(1)}, \lambda=-5 \alpha^{2} / 3+3 \beta / 2, \sigma=\operatorname{sign}(\lambda)$ and $C=C_{K} / 2$. Substituting the corresponding values of the parameters $\alpha$ and $\beta$, we obtain that for soft potentials $\lambda<0$ and then $\sigma=-1$, while for hard potentials $\lambda>0$

$\overline{2}$ When $\alpha=0$, coupling to higher harmonics appears only to order $\epsilon^{5}$ and higher. In particular, for the quartic potential only odd harmonics couple to each other. 
and $\sigma=1$. Actually both cases $\sigma= \pm 1$ are formally equivalent at this order of the expansion, since by changing $\psi_{n}$ into $(-1)^{n} \psi_{n}^{*}$ we get a new equation for $\left\{\psi_{n}\right\}$ which has the same form as Eq. (19) but with $\delta$ replaced by $(4 C-\delta)$, $\sigma$ by $-\sigma$ and $C$ is unchanged. So within the DNLS approximation we can assume for convenience $\sigma=-1$, corresponding to a soft potential, without loss of generality.

\section{$3 \quad$ Standing waves}

\subsection{Standing waves represented as map trajectories}

SWs in the nonlinear KG model (5) can be searched as time-reversible and time-periodic solutions $\left\{u_{n}(t)\right\}$ with frequency $\omega_{b}$ as in Eq. (10). The Fourier coefficients $w^{(p)}$ of the time-periodic function $V^{\prime}(u(t))=\sum_{p} w^{(p)} \mathrm{e}^{\mathrm{i} p \omega_{b} t}$ define nonlinear functions $\left\{w^{(p)}\left(\left\{a^{(q)}\right\}\right)\right\}$ of the Fourier coefficients $\left\{a^{(q)}\right\}$ of $u(t)$. Then, Eq. (5) can be written as

$$
-p^{2} \omega_{b}^{2} a_{n}^{(p)}+w^{(p)}\left(\left\{a_{n}^{(q)}\right\}\right)-C_{K}\left(a_{n+1}^{(p)}+a_{n-1}^{(p)}-2 a_{n}^{(p)}\right)=0 .
$$

This equation defines a symplectic map

$$
\left(\left\{a_{n+1}^{(p)}\right\},\left\{a_{n}^{(p)}\right\}\right)=\tilde{\mathcal{S}}\left(\left\{a_{n}^{(p)}\right\},\left\{a_{n-1}^{(p)}\right\}\right),
$$

where the SWs should appear as bounded trajectories. Since this map operates in an infinite dimensional space, its study is quite uneasy and moreover the numerous theorems about symplectic maps available for finite systems do not hold for infinite systems. It is convenient to reduce the dimensionality of this map, which can be done using the DNLS approximation derived in Sec. 2.2 (generally valid in the limit of small-amplitude solutions and small inter-site coupling) to eliminate all $a_{n}^{(p)}$ with $p \neq 1$.

Since the SWs are time-periodic solutions, all the amplitudes $a_{n}^{(p)}$ of the Fourier expansion (10), and therefore all the $\psi_{n}$, will be time independent, and they can be chosen real by restricting to time-reversible solutions. Then, vanishing the left-hand side of Eq. (19) yields

$$
\delta \psi_{n}-\sigma \psi_{n}^{3}+C\left(\psi_{n+1}+\psi_{n-1}-2 \psi_{n}\right)=0 .
$$

This equation defines a nonlinear symplectic cubic map $\mathcal{S}$ in the 2-dimensional real plane, which recursively determines $\left(\psi_{n+1}, \psi_{n}\right)$ from $\left(\psi_{n}, \psi_{n-1}\right)$. With the 
change of scale $\psi_{n} \rightarrow \sqrt{C} \psi_{n}^{\prime}$, this map depends only on the parameter $\delta^{\prime} \equiv$ $\delta / C$ :

$$
\psi_{n+1}^{\prime}=\left(2-\delta^{\prime}\right) \psi_{n}^{\prime}+\sigma \psi_{n}^{\prime 3}-\psi_{n-1}^{\prime}, \quad \psi_{n}^{\prime}=\psi_{n}^{\prime} .
$$

As is well-known [24], this map exhibits a rich variety of orbits, including elliptic and hyperbolic fixpoints and periodic cycles, KAM tori, Aubry-Mather Cantor sets (cantori) $[15,13,26]$ and chaotic orbits (see Fig. 2). Since we are searching for nonlinear SWs in continuation of the linear SWs, only bounded trajectories which can be continued to zero amplitude by varying $\delta^{\prime}=\frac{\delta}{C}$ are of interest.

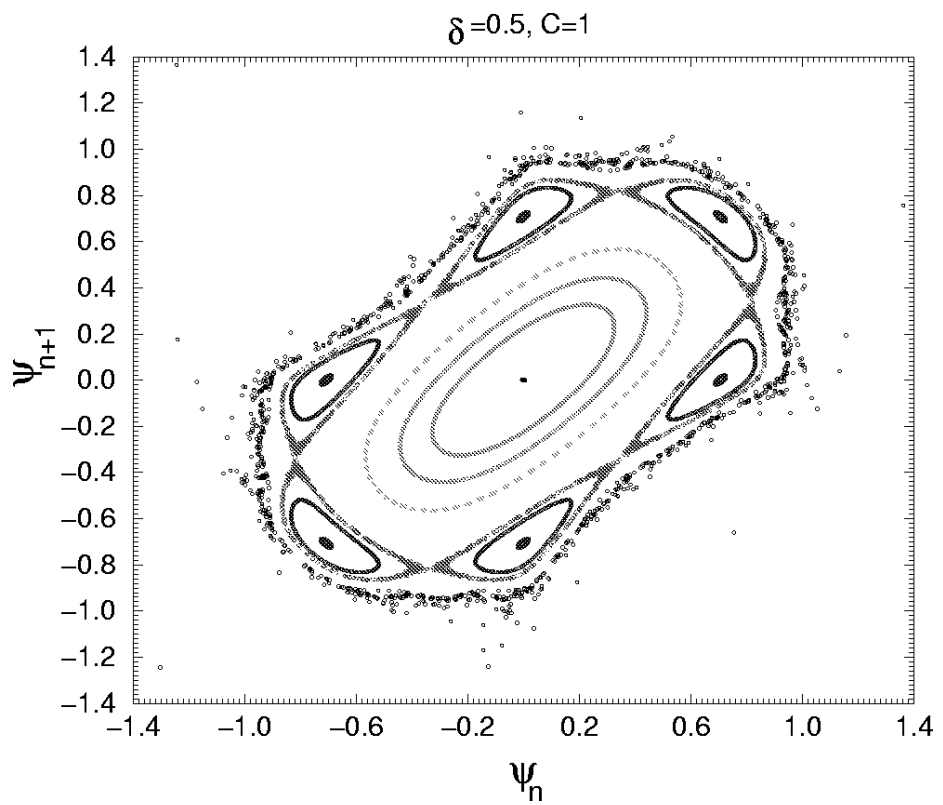

Fig. 2. The symplectic map $\mathcal{S}$ for $\delta^{\prime}=\frac{\delta}{C}=0.5(\sigma=-1)$. The visible chain of alternating elliptic and hyperbolic periodic points correspond to the two families of SWs with $Q=\pi / 3$.

When $\delta$ belongs to the phonon band, that is when $0 \leq \delta \leq 4 C$, the linearization of Eq. (22) has the solution $\psi_{n}=\epsilon_{s} \cos (Q n+\phi)$, where $Q$ fulfills

$$
\delta=4 C \sin ^{2} Q / 2 \equiv \delta_{0}(Q)
$$

which is equivalent to the KG dispersion relation $\omega_{b}^{2}=\omega_{l}^{2}(Q)=1+4 C_{K} \sin ^{2} \frac{Q}{2}$ (Eq. (8)). Then, the trivial fixpoint $F_{0}=(0,0)$ of the map $\mathcal{S}$ is linearly stable, that is elliptic with a rotation angle $Q$. This solution corresponds to a linear SW and the rotation angle is nothing but the wave vector $Q$ of the SW. When $\delta>\delta_{0}(\pi)=4 C$ the fixpoint $F_{0}=(0,0)$ becomes hyperbolic with reflection, while it becomes hyperbolic without reflection for $\delta<\delta_{0}(0)=0$. 
When $\delta^{\prime}=\frac{\delta}{C} \geq \frac{\delta_{0}(\pi)}{C}=4$ and $\sigma=-1$, it can be proven that the fixpoint $F_{0}=(0,0)$ corresponds to the only bounded trajectory of the map and that all other trajectories $\left\{\psi_{n}\right\}$ go to infinity for either positive or negative $n$ or for both. To demonstrate this assertion, let us assume that at some point a trajectory fulfills the condition $0 \neq\left|\psi_{n}\right| \geq\left|\psi_{n-1}\right|$. Then Eq. (23) yields that

$$
\left|\psi_{n+1}^{\prime}\right| \geq\left(\left|2-\delta^{\prime}-\psi_{n}^{\prime 2}\right|-1\right)\left|\psi_{n}^{\prime}\right|
$$

which for $\delta^{\prime} \geq 4$ yields $\left|\psi_{n+1}^{\prime}\right|>\left(\delta^{\prime}-3\right)\left|\psi_{n}^{\prime}\right| \geq\left|\psi_{n}^{\prime}\right|$ and thus implies recursively that $\left|\psi_{n}\right|$ goes to infinity for large positive $n$. If we have the reverse inequality $\left|\psi_{n}\right| \leq\left|\psi_{n-1}\right|$, we obtain identically the same result but for $n$ going to $-\infty$. Consequently, there are no spatially bounded time-periodic solutions with a frequency above the phonon band for a DNLS equation with a soft potential $(\sigma=-1)$. For a hard potential $(\sigma=1)$, the same argument excludes nontrivial bounded time-periodic solutions below the phonon band $\delta^{\prime} \leq 0$.

When $\delta^{\prime}=\frac{\delta}{C}<4$ for soft potentials (or, equivalently, $\delta^{\prime}>0$ for hard potentials), it can also be proven that the trajectories which escape too far from the origin go to infinity. Indeed, for $\sigma=-1$, if $\psi_{n}^{\prime 2}>4-\delta^{\prime}$ and $\left|\psi_{n}\right| \geq\left|\psi_{n-1}\right|$, the inequality (25) implies that $\psi_{n+1}^{\prime 2}>\psi_{n}^{\prime 2}>4-\delta^{\prime}$ and recursively that $\left|\psi_{n}\right|$ goes to $+\infty$ for $n \rightarrow+\infty$. A similar result holds for the reverse inequality $\left|\psi_{n}\right| \leq\left|\psi_{n-1}\right|$ but then $\left|\psi_{n}\right|$ goes to $+\infty$ for $n \rightarrow-\infty$. Nevertheless, when $\delta^{\prime}<4$ the nonlinear map $\mathcal{S}$ exhibits many bounded trajectories representing SWs. As mentioned above (see Fig. 2), some of them are chaotic but there are also periodic cycles, KAM tori and cantori for example around the elliptic fixpoint $F_{0}=(0,0)$.

As the nonlinear SWs we search for should be continuable to zero amplitude when varying $\delta^{\prime}=\frac{\delta}{C}$, the corresponding orbits should merge into the elliptic fixpoint $F_{0}$ for the particular value $\delta=\delta_{0}(Q)$. As suggested in [12], trajectories which have this property should be either periodic cycles or quasiperiodic trajectories. The quasiperiodic trajectories, corresponding to wave vectors $Q$ incommensurate with $2 \pi$, should, for most irrational values of $Q / 2 \pi$, appear as KAM tori with rotation angle $Q$. Thus, given a rotation angle $Q$, there is at least one trajectory which appears with this rotation angle at the elliptic point $F_{0}$ for $\delta=\delta_{0}(Q)$, and which for $\delta$ close to $\delta_{0}(Q)$ is located close to $F_{0}$. This trajectory, which for rational $Q / 2 \pi=r / s$ is a periodic cycle with period $s$ and for irrational $Q / 2 \pi$ generally a KAM torus, represents a small amplitude SW with wave vector $Q$. For a general potential (soft or hard), the coefficient $\sigma$ of the cubic term in the DNLS equation (22) determines the behaviour of the tori around the elliptic fixpoint $F_{0}$. For a soft potential $(\sigma<0)$ the rotation angle for orbits rotating around $F_{0}$ increases with increasing radius, and in particular for KAM tori with small radius $\epsilon_{s}$ the rotation angle can be 
calculated to lowest order in $\epsilon_{s}$ as

$$
\cos Q \simeq 1-\frac{\delta^{\prime}}{2}+\frac{3 \sigma \epsilon_{s}^{2}}{8 C}
$$

Since the map $\mathcal{S}$ depends continuously on the map parameter $\delta^{\prime}$, the periodic cycles, KAM tori and cantori of $\mathcal{S}$ can be continued as a function of $\delta^{\prime}$, and its orbits at fixed rotation angle depend continuously on $\delta^{\prime}[12]$. Then, as $\delta_{0}(Q)$ is a monotonously increasing function of $Q$, we can conclude that SWs with wave number $Q$ exist only for $\delta \leq \delta_{0}(Q)$. Similarly, for a hard potential $(\sigma>0)$ the rotation angle decreases with increasing radius and SWs exist only for $\delta \geq \delta_{0}(Q)$

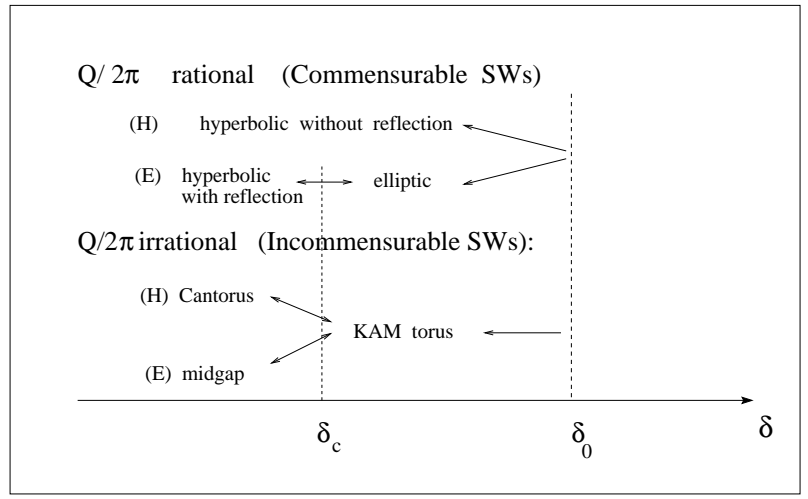

Fig. 3. Evolution of the trajectories for variable $\delta^{\prime}$ and fixed wave vector $Q(\sigma=-1)$.

To study the evolution of the trajectories representing SWs (for $\sigma=-1$ ) when varying the map parameter $\delta^{\prime}$, we thus start from the linear limit at $\delta=\delta_{0}(Q)$ corresponding to the fixpoint $F_{0}$ and decrease the value of $\delta$. This evolution is schematically illustrated in Fig. 3. For a rational value of $\frac{Q}{2 \pi}=r / s$ ( $r$ and $s$ irreducible integers), which corresponds to SWs commensurable with the lattice periodicity, there are two families of SWs corresponding to the two $s$-periodic cycles which bifurcate in pair from $F_{0}$ at the linear limit $\delta=\delta_{0}(Q)$. We will call these $s$-periodic cycles ' $h$-cycle' and 'e-cycle', respectively. The $h$-cycles are hyperbolic without reflection for all $\delta<\delta_{0}(Q)$. By contrast the $e$-cycles are elliptic trajectories for $\delta$ close to $\delta_{0}(Q)$, but decreasing the value of $\delta$ they become hyperbolic with reflection below a critical value $\delta=\delta_{c}(Q)$ which can be calculated accurately numerically e.g. using Greene's residue [27].

When $Q / 2 \pi$ is a generic irrational number (i.e., not a Liouville number), the trajectories representing the spatially quasiperiodic, incommensurate, SWs emerge from $F_{0}$ as the KAM torus with rotation angle $Q$, which is unique apart from a phase shift. Decreasing the value of $\delta$ below the critical value

$\overline{3}$ This follows e.g. from Eq. (A.13) in the Appendix using Eq. (43). 

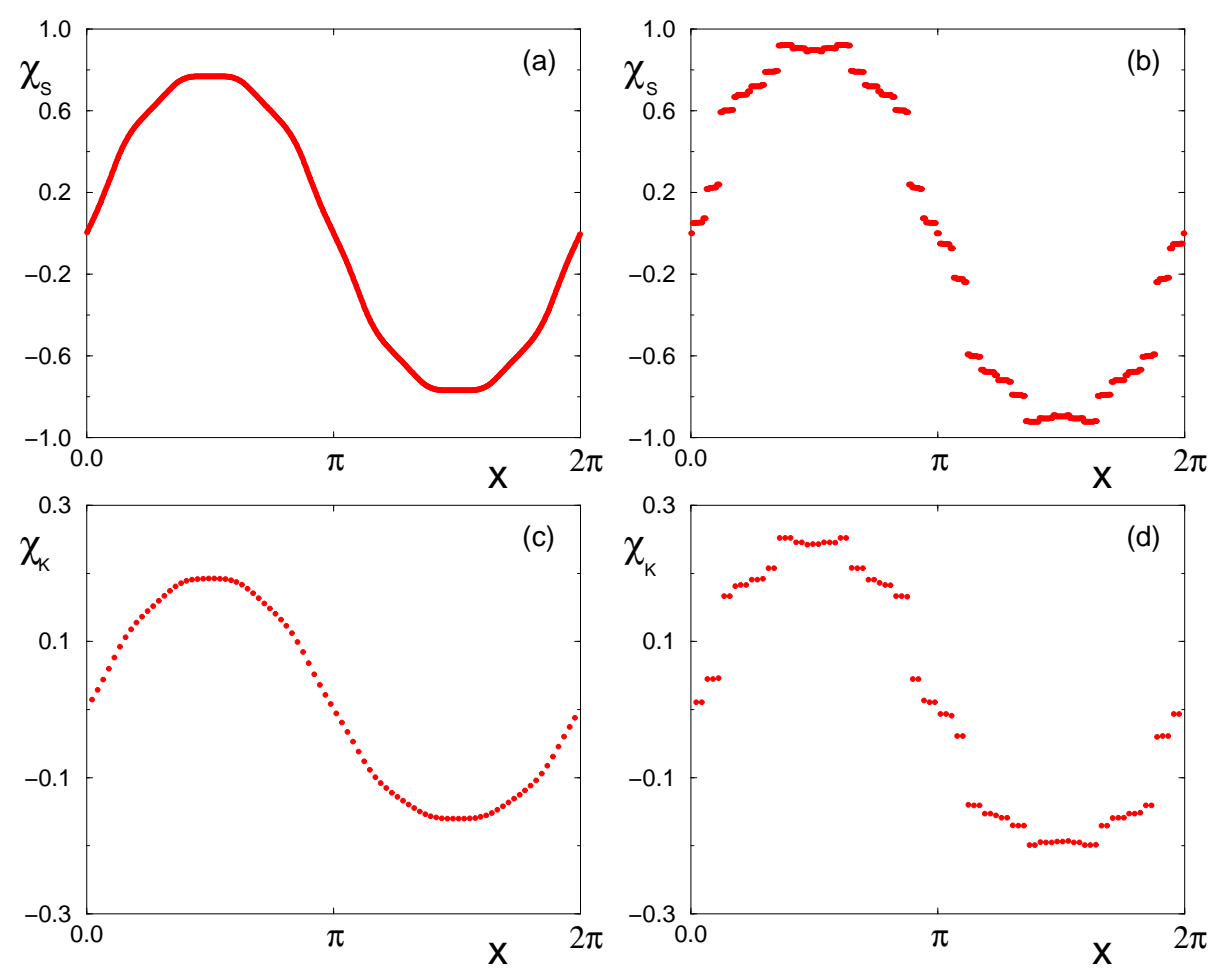

Fig. 4. (a) and (b): Hull functions $\chi_{S}(x)$ for SWs in a DNLS model (22) with $Q / 2 \pi=233 / 610 \simeq(3-\sqrt{5}) / 2 \equiv \sigma_{G}$ and (a) $\delta^{\prime}=3.0$ resp. (b) $\delta^{\prime}=2.8(C=1)$. The analyticity is found to be broken at $\delta^{\prime} \simeq 2.92$ for $Q / 2 \pi=\sigma_{G}$. (c) and (d): Hull functions $\chi_{K}(x)$ for SWs in a KG chain (5) with a Morse potential, with $C_{K}=0.05, Q / 2 \pi=34 / 89 \simeq \sigma_{G}$ and (c) $\omega_{b}=1.072$ resp. (d) $\omega_{b}=1.065$. The breaking of analyticity occurs at $\omega_{b} \simeq 1.070$ for $Q / 2 \pi=\sigma_{G}$.

$\delta=\delta_{c}(Q)$, the KAM torus bifurcates into two types of trajectories, the cantorus and its associated 'midgap' trajectory [28]. This midgap trajectory consists of isolated, nonrecurrent points in the gaps of the cantorus with rotation angle $Q$, to which it is homoclinic. As the KAM torus is an analytic trajectory while the cantorus and the midgap trajectory are non-analytic, the transition at the critical point $\delta_{c}$ corresponds to a transition by breaking of analyticity (TBA) $[13,26]$. The transition point $\delta_{c}(Q)$ is determined as the limit of the critical values $\delta_{c}\left(Q^{(k)}\right)$ for a sequence of periodic e-cycles whose rotation angles $Q^{(k)}$ are obtained from successive rational approximants of $Q / 2 \pi$ [27]. With this transition the two families of non-analytic incommensurate $S W s$, represented by the cantori and the midgap trajectories, respectively, merge into the unique family of analytic $S W$ defined by the KAM tori for $\delta \geq \delta_{c}(Q)$. Defining the hull (or envelope) function $\chi_{S}(x)$ for the SW as

$$
\psi_{n}=\chi_{S}(Q n+\phi)
$$

which gives the shape of the wave rescaled inside one period $2 \pi$, the TBA for this hull function when decreasing $\delta$ is shown in Figs. 4(a) and 4(b). As the 
model symmetry implies two axis of symmetry for the KAM tori (see Fig.2), the hull function $\chi_{S}(x)$ can be chosen with the symmetries $\chi_{S}(x)=-\chi_{S}(-x)$ and $\chi_{S}(x)=-\chi_{S}(x+\pi)$. The nonanalyticity of the SW hull functions for large negative $\delta$ makes a description in terms of multibreathers natural, as will be discussed in Sec. 3.2.

As the trajectories of the map $\mathcal{S}$ represent approximate solutions for the KG model in the limit of small coupling, we should expect to find this TBA also for SWs in the KG system (5). We can calculate numerically exact KG SW solutions using a Newton scheme as described, e.g., in $[11,7,29]$ (see also Secs. 3.2 and 5). Defining the hull function $\chi_{K}(x)$ for these SWs as $u_{n}(0)=\chi_{K}(Q n+\phi)$ and continuing these solutions versus $\omega_{b}$ to zero amplitude, a TBA is found for the function $\chi_{K}(x)$ as illustrated in Figs. 4(c) and 4(d). (Note however that for a non-symmetric potential such as the Morse potential, the hull function does not in general have the above-mentioned symmetries, i.e., $\chi_{K}(x) \neq-\chi_{K}(-x)$ and $\chi_{K}(x) \neq-\chi_{K}(x+\pi)$.)

\subsection{Standing waves as multibreather solutions}

When the SW frequency $\omega_{b}$ is below the phonon spectrum for a soft potential (that is $\left.\omega_{b}<1\right)$ or above for a hard potential $\left(\omega_{b}>\sqrt{1+4 C_{K}}\right)$ and close enough to the anticontinuous limit $C_{K}=0$, the SW solutions can be identified as multibreathers [4] with period $T_{b}=\frac{2 \pi}{\omega_{b}}$. These are oscillatory states composed by nonlinear combinations of several time-periodic and spatially localised breathers in a single nonlinear system [4,30]. These multibreather solutions are characterized by a coding sequence $\tilde{\sigma}_{n}[4,12]$ which defines the particular oscillatory state of the oscillator $n$ in the case of zero coupling. As for general time-reversible time-periodic solutions with period $T_{b}$ each individual oscillator can oscillate with frequency $j \omega_{b}$ ( $j$ integer) with two possible choices for the phase, we can, at $C_{K}=0$, characterize all such solutions for the whole system by the following coding sequence:

- $\tilde{\sigma}_{n}=0$ when the oscillator $n$ is at rest;

- $\tilde{\sigma}_{n}=+1$ when the oscillator $n$ oscillates with a finite amplitude at frequency $\omega_{b}$ and in phase with the chosen reference solution of the single oscillator;

- $\tilde{\sigma}_{n}=-1$ when the oscillator $n$ oscillates at frequency $\omega_{b}$ in antiphase with the chosen reference solution;

- $\tilde{\sigma}_{n}=+2$ when the oscillator $n$ oscillates in phase at frequency $2 \omega_{b}$;

- ...

4 We will use the term multibreather to refer to combinations of either finitely or infinitely many individual discrete breathers. 


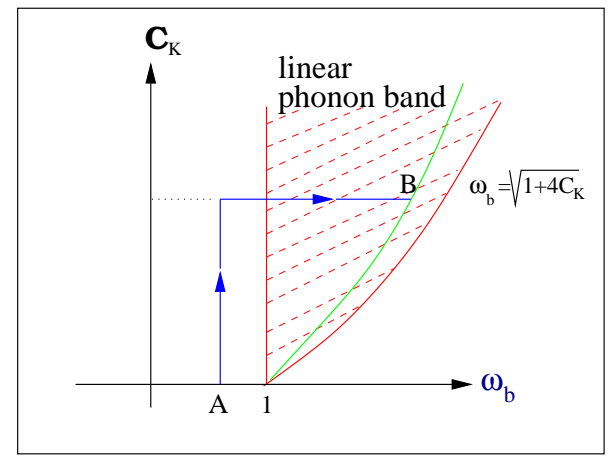

Fig. 5. Continuation of the multibreather solution with wave vector $Q$ into the linear phonon band for a soft potential, from the anti-continuous limit $C_{K}=0$ to the linear limit $\omega_{b}=\sqrt{1+4 C_{K} \sin ^{2} \frac{Q}{2}}$ illustrated by the gray line. (For a hard potential, the continuation starts at $\omega_{b}>1$ and can be performed at constant frequency.)

These solutions are proven to be continuable when $C_{K}$ varies from the anticontinuous limit up to a finite value of the coupling. At this value, which depends in general on the solution in consideration, bifurcations are found and the solution can be lost. This will be numerically investigated in more details for SWs in Section 5. We consider now only the continuable regime of the multibreathers, and in particular we search among these multibreather states the solutions which are in continuation of the linearized SWs at low amplitude. For that purpose, we have to find an appropriate coding sequence $\left\{\tilde{\sigma}_{n}\right\}$ such that the multibreather which exists at $C_{K} \neq 0$ can be continued versus its frequency inside the phonon band, as illustrated in Fig. 5. A similar problem has already been studied in [11] for a disordered model. Actually, it was found that most of the multibreather solutions undergo bifurcations and disappear before their frequency enters the phonon spectrum, although some of them can be continued while their frequency belongs to the phonon spectrum. For this last type of solutions an ansatz was found for calculating specifically the multibreather states which are continuable as linearized harmonic modes. However, the method used in [11] was explicitly based on the property that the phonon spectrum is discrete with localized eigenmodes. In our translationally invariant model this property is not fulfilled so that we cannot use the ansatz proposed in [11]. There are nevertheless two linearized solutions which can be trivially obtained by continuation of multibreather states, namely the $Q=0$ phonon from the multibreather with code $\ldots+1+1+1+1+1+1+1+1 \ldots$, and the $Q=\pi$ phonon from the multibreather with code ... $+1-1+1-1+1-1+1-1 \ldots$ Our problem is to find the ansatz which gives the coding sequence for a general SW with wave vector $Q$. Once this code is obtained, it is possible to calculate the nonlinear SW for different values of the frequency and coupling by numerical continuation.

The method we propose to find the ansatz consists in passing from the KG model (5) to the DNLS model (19) (where we for simplicity assume $\sigma=-1$ 
without loss of generality) by using the small-amplitude (and generally also small-coupling) approximation (Sec. 2.2), so to obtain the SW representations as trajectories of the symplectic map $\mathcal{S}(23)$. As the DNLS map $\mathcal{S}$ only depends on the parameter $\delta^{\prime}=\frac{\delta}{C}$, the anticontinuous limit $C=0$ for fixed frequency $\delta<0$ is in the DNLS description equivalent to the large-amplitude limit $\delta \rightarrow-\infty$ for fixed coupling $C$. Thus, continuing the SW trajectories to this limit, they can be described as multibreathers and associated to a coding sequence. As for $C=0$ the stationary solutions to Eq. (22) can only take the values $\psi_{n}= \pm \sqrt{-\delta}$ and $\psi_{n}=0$, the code will be defined as $\tilde{\sigma}_{n}=+1$ when $\psi_{n}=\sqrt{-\delta}, \tilde{\sigma}_{n}=-1$ when $\psi_{n}=-\sqrt{-\delta}$ and $\tilde{\sigma}_{n}=0$ when $\psi_{n}=0$. These codes can then be used as initial conditions in an iterative Newton scheme to calculate numerically the SWs in the original KG model (5) to any desired accuracy.

By continuing the SW solutions calculated for the DNLS map to large negative $\delta^{\prime}$ we guessed and tested, by inspecting the map trajectories, the following ansatz for their coding sequences

$$
\tilde{\sigma}_{n}=\chi_{0}(Q n+\phi)
$$

where $\tilde{\sigma}_{n}$ can be quasi-periodic or periodic, depending on whether $Q / 2 \pi$ (with $0 \leq Q \leq \pi)$ is irrational or rational, and the hull function $\chi_{0}(x)$ is a $2 \pi$ periodic odd function defined for $x \in[-\pi, \pi]$ as

$$
\chi_{0}(x)=\left\{\begin{array}{l}
1 \quad \text { for } \quad(\pi-Q) / 2 \leq x \leq(\pi+Q) / 2 \\
-1 \quad \text { for } \quad-(\pi+Q) / 2 \leq x \leq-(\pi-Q) / 2 . \\
0 \quad \text { elsewhere }
\end{array}\right.
$$

This function $\chi_{0}(x)$, rescaled by a factor $\sqrt{-\delta}$, is the limit for $C=0$ of the hull function $\chi_{S}(x)$ introduced in Section 3.1.

This method for generating the code can be visualised by a graphical circle construction (see Fig. 6). To construct the code for a nonlinear SW with wave vector $Q$, the circle is divided in four parts to which the values $0,+1$ or -1 are associated. Rotating around the circle by the angle $Q$ yields the code sequence of the SW. The value of the phase $\phi$, corresponding in the circle construction to the initial point for the rotation, has particular importance. For all phases $\phi \neq \phi_{m} \equiv \pm(\pi-Q) / 2-m Q$ ( $m$ integer), the coding sequence generated does not contain any consecutive codes +1 or -1 . We call these SWs 'type $H$ ' and, with the terminology used in Sec. 3.1 , they correspond either to the $h$-cycles

5 For a hard potential $(\sigma=+1)$, Eqs. (28)-(29) yield the SWs with wave vector $\pi-Q$ via the transformation $\tilde{\sigma}_{n} \rightarrow(-1)^{n} \tilde{\sigma}_{n}$. 


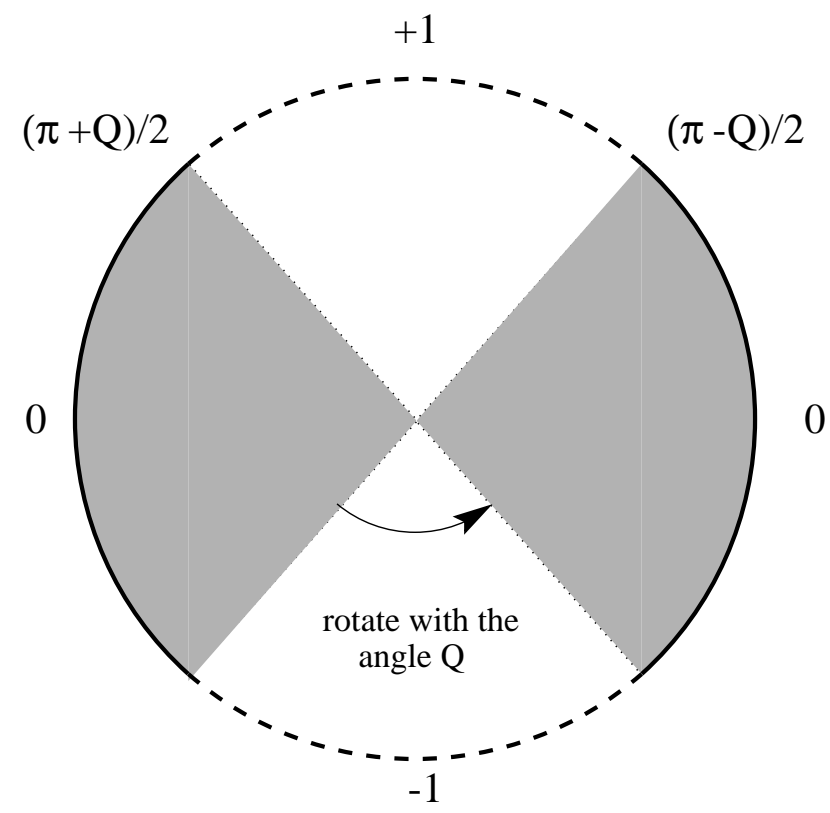

Fig. 6. Graphical representation of the generation of the code sequence for a general nonlinear SW with wave vector $Q$.

(for rational $Q / 2 \pi$ ) or to the cantori (for irrational $Q / 2 \pi$ ). For the particular phases $\phi=\phi_{m}, x=Q m+\phi$ is at a discontinuity point of $\chi_{0}(x)$, and, as a consequence, $\tilde{\sigma}_{n}$ has two consecutive +1 (or -1$)$. These SWs, called 'type $E$ ', correspond to the $e$-cycles (for rational $Q / 2 \pi$ ) or to midgap trajectories (for irrational $Q / 2 \pi$ ). As will be seen in Sec. 4, this characteristic of the code will imply important consequences for the stability of nonlinear SWs. We note that the coding sequences for SWs of type $E$ will always be symmetric around a bond center, and can therefore be chosen to fulfill $\tilde{\sigma}_{n}=\tilde{\sigma}_{-n+1}$ by choosing $\phi=(\pi-Q) / 2$. In the special cases when $Q=\frac{2 k+1}{2 k^{\prime}+1} \pi\left(k, k^{\prime}\right.$ integers), the codes for type $E \mathrm{SWs}$ will in addition be antisymmetric around some lattice site, and can therefore alternatively be chosen to fulfill $\tilde{\sigma}_{n}=-\tilde{\sigma}_{-n}$ by choosing $\phi=0$. The coding sequences for type $H$ SWs are antisymmetric around a lattice site except when $Q=\frac{2 k+1}{2 k^{\prime}+1} \pi$, in which case they are antisymmetric around a bond center. In the former case we can choose $\phi=0$ so that $\tilde{\sigma}_{n}=-\tilde{\sigma}_{-n}$, while in the latter case $\tilde{\sigma}_{n}=-\tilde{\sigma}_{-n+1}$ by choosing $\phi=-Q / 2$.

Finally, we remark that the construction (29) to generate the SW coding sequence can, for type $H$ SWs with $Q$ close to $\pi$, be regarded as a periodic or quasiperiodic repetition of defects or discommensurations added to the $Q=\pi$ phonon, where each discommensuration consists of inserting one additional site with code $\tilde{\sigma}_{n}=0$ in the coding sequence for the $Q=\pi$ phonon. In the limit $Q \rightarrow \pi$, the distance between the discommensuration sites goes to infinity, so that we recover the case of one single defect site which was studied for the DNLS model in [18] and shown to correspond to the discrete counterpart of the dark NLS soliton. As will be discussed later, this point of view is useful in order to understand the origin of the instabilities of the SWs for $Q>\pi / 2$. 


\section{Stability of nonlinear standing waves in the DNLS approxima- tion}

We now turn to the main topic of this paper, namely the characterization of the dynamical stability properties of nonlinear SWs. We will here first consider small-amplitude solutions to the KG equation (5) with small $C_{K}$, so that the DNLS approximation (19) is well justified. In the next section we will study numerically the original KG model for larger coupling to investigate the limit of validity for the DNLS approximation. Here, we assume for convenience $\sigma=$ -1 without loss of generality (Sec. 2.2). With the substitution $\psi_{n} \rightarrow \psi_{n}+\epsilon_{n}(t)$, where $\epsilon_{n}$ is small, the linearization of Eq. (19) yields a standard Hill equation for $\epsilon_{n}[31]$,

$$
i \dot{\epsilon}_{n}=\delta \epsilon_{n}+2\left|\psi_{n}\right|^{2} \epsilon_{n}+\psi_{n}^{2} \epsilon_{n}^{*}+C\left(\epsilon_{n+1}+\epsilon_{n-1}-2 \epsilon_{n}\right) .
$$

A solution $\left\{\psi_{n}\right\}$ of Eq. (19) is said to be linearly stable when there is no solution $\left\{\epsilon_{n}(t)\right\}$ of Eq. (30) which diverges exponentially at large time.

In the case when $\psi_{n}$ is a real and time-independent solution of Eq. (19), the general solution of (30) can be searched as linear combinations of solutions with the form

$$
\epsilon_{n}(t)=a_{n} \mathrm{e}^{\mathrm{i} \omega t}+b_{n}^{*} \mathrm{e}^{-\mathrm{i} \omega t}
$$

where the eigenfrequencies $\omega=\omega_{s}$ and eigenmodes $\left\{a_{n}, b_{n}\right\}$ are determined by the eigenequation

$$
\begin{gathered}
\left(2 C-\delta-2 \psi_{n}^{2}\right) a_{n}-C\left(a_{n+1}+a_{n-1}\right)-\psi_{n}^{2} b_{n}=\omega_{s} a_{n} \\
\psi_{n}^{2} a_{n}+C\left(b_{n+1}+b_{n-1}\right)-\left(2 C-\delta-2 \psi_{n}^{2}\right) b_{n}=\omega_{s} b_{n} .
\end{gathered}
$$

Then, we note that if $\omega_{s}$ is an eigenvalue for the eigenvector $\left\{a_{n}, b_{n}\right\}$, then $-\omega_{s}$ and $\omega_{s}^{*}$ are eigenvalues for the eigenvectors $\left\{b_{n}, a_{n}\right\}$ and $\left\{a_{n}^{*}, b_{n}^{*}\right\}$, respectively $\square$. As a result, linear stability is achieved if and only if all the eigenvalues of Eq. (32) are real. Thus, for a large but finite system with $N$ sites, we should have $2 N$ real eigenvalues $\omega_{s}$ for a linearly stable solution.

It is convenient to redefine new variables $U_{n}=a_{n}+b_{n}$ and $W_{n}=a_{n}-b_{n}$, so that Eqs. (32) become [31]

$$
\begin{aligned}
& \mathcal{L}_{0} W_{n} \equiv\left(2 C-\delta-\psi_{n}^{2}\right) W_{n}-C\left(W_{n+1}+W_{n-1}\right)=\omega_{s} U_{n} \\
& \mathcal{L}_{1} U_{n} \equiv\left(2 C-\delta-3 \psi_{n}^{2}\right) U_{n}-C\left(U_{n+1}+U_{n-1}\right)=\omega_{s} W_{n} .
\end{aligned}
$$

6 The exponential of this operator is symplectic. 
The operators $\mathcal{L}_{0}$ and $\mathcal{L}_{1}$ are self-adjoint, but not the whole operator $\mathcal{L} \equiv$ $\left(\begin{array}{rr}0 & \mathcal{L}_{0} \\ \mathcal{L}_{1} & 0\end{array}\right)$. Note that $\omega_{s}=0$ is always an eigenvalue of $\mathcal{L}$ for $U_{n} \equiv 0$, since the first equation in (33) then has the explicit solution $W_{n}=\psi_{n}$, corresponding to the time-independent phase mode $\epsilon_{n}=i \psi_{n}$ resulting from the invariance of the model under global phase rotations. Moreover, for an incommensurate SW with analytic hull function (27) there is also an additional solution to (33) with $\omega_{s}=0$ and $W_{n} \equiv 0$, namely the sliding mode solution $U_{n}=\partial \chi_{S} / \partial \phi$ to the second equation, resulting from the invariance of analytic SWs under spatial translations. See also Appendix A.2 for a further discussion of the spectrum of the operators $\mathcal{L}_{0}$ and $\mathcal{L}_{1}$.

We also define the Krein signature [32,4] associated with a pair of eigenvalues $\pm \omega_{s}$ as [33]

$$
\mathcal{K}\left(\omega_{s}\right)=\operatorname{sign} \sum_{n}\left[\left|a_{n}\right|^{2}-\left|b_{n}\right|^{2}\right]=\operatorname{sign} \sum_{n}\left[U_{n} W_{n}^{*}+W_{n} U_{n}^{*}\right],
$$

where $\left\{a_{n}, b_{n}\right\}$ and $\left\{U_{n}, W_{n}\right\}$ are the eigenvectors of (32) resp. (33) with eigenvalue $+\omega_{s}$. In general, when varying the parameter $\delta^{\prime}$, instabilities may occur only through collisions between eigenvalues with different Krein signatures.

When $\delta<0$ and $C \rightarrow 0$ (anticontinuous limit), $\psi_{n}^{2}=-\delta$ or 0 (codes $\tilde{\sigma}_{n}= \pm 1$ or 0 , respectively), and all eigenfrequencies of (33) are easily seen to be real. For a finite system with $P$ sites where $\left|\tilde{\sigma}_{n}\right|=1$ and $Z=N-P$ remaining sites where $\tilde{\sigma}_{n}=0$, there are $2 P$ eigenvalues degenerated at $\omega_{s}=0$, while the remaining eigenvalues are located as $Z$ pairs at $\omega_{s}= \pm \delta$. Increasing the value of the coupling, the eigenvalues will move losing their degeneracy and, if a pair of eigenvalues goes out in the imaginary plane, the nonlinear SW may become unstable. In particular, when $C$ is increased from zero, the eigenvalues at $\omega_{s}= \pm \delta$ (which all have Krein signature +1 ) will move only along the real axis causing no instabilities for the SWs. On the other hand, the behaviour of the eigenvalues originating from $\omega_{s}=0$ will be different for the $E$-type and $H$ type SWs (as defined in Sec. 3.2). As the coding sequences for the $E$-type SWs contain pairs of consecutive +1 (or -1 ) and the anharmonicity is soft, it follows from a general result [4] that some pairs of eigenvalues originally at $\omega_{s}=0$ leave the real axis and move out on the imaginary axis as soon as $C \neq 0$. As a consequence, the corresponding SWs become dynamically unstable for $\delta<0$ and small $C$. Moreover, we find numerically that this instability persists for all $\delta<\delta_{0}(Q)$, so that SWs of type $E$ are unstable for any nonvanishing amplitude $\square$ By contrast, for $H$-type SWs, for every site with code $\tilde{\sigma}_{n}=1$ the

7 This non-oscillatory instability is also proven in the Appendix for commensurate SWs of type $E$ in the small-amplitude limit. 
nearest surrounding sites with nonzero code have $\tilde{\sigma}_{n}=-1$, and reciprocally (i.e., there is a phase shift of $\pi$ between any two consecutive sites with nonzero oscillation at $C=0$, neighboring or not). Then, the theory of effective action developed in Ref. [4] yields, with similar calculations as in [34,18], that all eigenvalues originating from $\omega_{s}=0$ (having Krein signature -1 opposite to those originating from $\omega_{s}= \pm \delta$ ) move away from the origin along the real axis, except one pair remaining at $\omega_{s}=0$ (with Krein signature 0) corresponding to the global phase invariance. Thus, the stability of these $S W s$ is preserved for $C$ not too large (c.f. Fig. 7 (a)).

\subsection{Commensurate $S W s$}

For commensurate SWs with $Q=\pi r^{\prime} / s^{\prime}\left(r^{\prime}\right.$ and $s^{\prime}$ irreducible integers) in an infinite system, the lattice periodicity of $\psi_{n}^{2}$ in (33) implies that the degenerate eigenvalues on the real axis form bands as $C$ is increased from zero (see Fig. 7). As there are $s^{\prime}-r^{\prime}$ sites with code $\tilde{\sigma}_{n}=0$ in a lattice period of $s^{\prime}$ sites, there will be $s^{\prime}-r^{\prime}$ symmetric pairs of bands with Krein signature +1 originating from $\omega_{s}= \pm \delta$ and separated by gaps. The remaining eigenvalues form $r^{\prime}$ symmetric pairs of bands with Krein signature -1 originating from $\omega_{s}=0$. As the eigenvalue $\omega_{s}=0$ always remains in the spectrum, there is no gap at zero which thus always remains at a band edge. For fixed $C$ and increasing $\delta$, the bands originating from $\omega_{s}=0$ and those from $\omega_{s}= \pm \delta$ broaden and the gap separating these two classes of bands will shrink. At a critical value $\delta=$ $\delta_{K}(Q)$ this middle gap shrinks to zero and, as shown in Fig. 7(b), symmetric pairs of eigenfrequencies with opposite Krein signature start to collide and move out from the real axis into the complex plane. Thus, oscillatory (Krein) instabilities (i.e., caused by complex eigenvalues) of the nonlinear SW are induced.
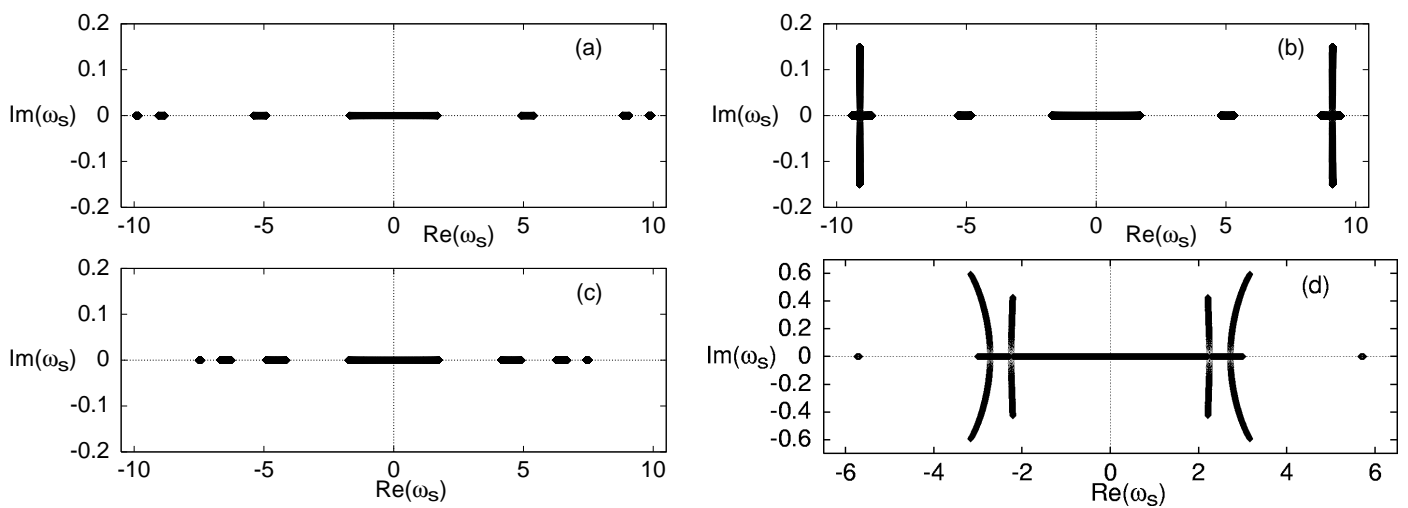

Fig. 7. Eigenvalues of Eq. (33) for a type $H$ SW with $Q=3 \pi / 4$ and (a) $\delta^{\prime}=-8.0$, (b) $\delta^{\prime}=-7.5$, (c) $\delta^{\prime}=-4.5$ resp. (d) $\delta^{\prime}=-0.5(C=1)$.

This instability mechanism is similar to that previously observed [18] for the 'discrete dark solitons', where the anticontinuous coding sequence consists of 
one single zero-amplitude 'defect' site added to a background wave with $Q=\pi$ (the latter being always stable [8]). In fact, as was mentioned in Sec. 3.2, for $Q$ close to $\pi$ the SWs generated from (29) can be viewed as a periodic (or quasi-periodic for incommensurate SWs) repetition of defects or discommensurations added to the stable wave with $Q=\pi$. Thus, the SW can be regarded as a lattice of weakly interacting discommensurations ("dark solitons"), each of which shows an oscillatory instability due to a resonance between a translational mode localized at the discommensuration and extended modes [18]. This oscillatory instability detected numerically for the DNLS model can also be confirmed by a direct numerical Floquet analysis of the KG Eq. (5) (see Section 5).

Increasing further $\delta$ above the critical value $\delta_{K}(Q)$, bands continue to overlap and generate new instabilities. For many commensurate values of $Q$, it is found that when $\delta$ increases beyond $\delta_{K}(Q)$, the SW always remains unstable until it vanishes with zero amplitude at the linear limit $\delta=\delta_{0}(Q)$. However, for some rational values $Q / 2 \pi=\frac{r}{s}$ with small $s$, in some intervals of $\delta$ the band overlap disappears, and consequently the stability is temporarily regained (e.g. for $Q=3 \pi / 4$, the $H$-type SW is stable for $-4.9 \lesssim \delta^{\prime} \lesssim-3.9$, see Fig. $\left.7(\mathrm{c})\right)$. In any case, for all commensurate SWs with $Q \neq \pi$, there will always be a final interval $\delta_{1}(Q) \leq \delta<\delta_{0}(Q)$ where bands with opposite Krein signature overlap (Fig. 7). As a consequence all commensurate $S W s$ with $Q \neq \pi$ are unstable for small but nonzero amplitude. This result can be simply understood from the remark that at the linear limit $\delta=\delta_{0}(Q)$ where $\psi_{n}^{2}=0$, the frequencies of the (gapless) spectrum of (33) are given by

$$
\omega_{s}= \pm 2 C|\cos Q-\cos q|
$$

$0 \leq q<2 \pi$, and the corresponding Krein signatures are given by $\operatorname{sign}(\cos Q-$ $\cos q)$. Thus, in the linear limit there is an interval around $\omega_{s}=0$ where eigenvalues with opposite Krein signature overlap, although there is of course no instability since there is no coupling between modes with opposite Krein signature in this limit. By a continuity argument, since in the commensurate case when $\psi_{n}^{2} \neq 0$ a finite number of bands with nonzero widths appear for $\delta<\delta_{0}(Q)$, bands with opposite Krein signature would, assuming the absence of mode coupling, continue to overlap on the real axis close to $\omega_{s}=0$ until $\delta$ reaches some value $\delta_{1}(Q)$ which must be strictly smaller than $\delta_{0}(Q)$. However, as in general there is coupling (i.e., resonances) between modes of different Krein signature, the overlapping bands move out in the complex plane and instabilities result. This is proven in the Appendix using perturbation theory and the method of 'band analysis' outlined in Sec. 4.2 below.

Let us also remark that in the case of finite-size systems (e.g., with periodic boundary conditions), the eigenvalues $\omega_{s}$ will evidently be separated in a discrete spectrum without forming continuous bands. The initial scenario when 
increasing $\delta^{\prime}$ from $-\infty$ will however be qualitatively the same as described above, and oscillatory instabilities will result similarly as illustrated in Fig. 7(b) (although the bands are replaced by discrete eigenvalues). However, the above argument showing instability close to the linear limit for infinite systems is obviously not valid for finite systems, as there are no continuous bands around $\omega_{s}=0$ but only discrete eigenvalues. Thus, overlap (resonances) can be avoided and, in fact, we find that for finite systems stability will generally be regained in some interval close to the linear limit. The scenario by which the stability is recovered is through 'reentrant instabilities' similarly as in $[17,18]$, and appears in an analogous way also for the KG model as will be described in Sec. 5.

\subsection{Incommensurate $S W s$}

For incommensurate SWs $(Q / 2 \pi$ irrational), oscillatory instabilities will occur at some critical value $\delta_{K}(Q)$ just as for the commensurate case described above, although the spectrum of (33) now has a Cantor set structure with infinitely many gaps. However, for small-amplitude (analytic) incommensurate SWs, the Cantor set nature of the spectrum (with 'bands' of zero width) implies that the argument used in the previous section cannot be used to prove the instability of the nonlinear SWs close to the linear limit, since it would only yield $\delta_{1}(Q)=\delta_{0}(Q)$. Indeed, numerical analysis of finite-size approximants to incommensurate analytic SWs with $|Q|>\pi / 2$ typically yields a scenario with reentrant instabilities which become very weak and practically invisible for $\delta$ close to $\delta_{0}(Q)$, and extrapolating this scenario to infinite systems could lead to the (erroneous) guess that analytic SWs with $|Q|>\pi / 2$ could be stable for some $\delta$ (possibly constituting a Cantor set as observed in disordered systems [11]). This scenario would mean that in the absence of mode coupling, the (Cantor-like) subspectrum of (33) with positive Krein signature would, for some $\delta$, exactly fit into the gaps of the other (Cantor-like) subspectrum with negative Krein signature, and thus instabilities could be avoided. However, as $\delta \rightarrow \delta_{0}(Q)$ both subspectra acquire full measure, and if their measure is assumed to be continuous with respect to $\delta$ overlap cannot be avoided for $\delta$ close enough to $\delta_{0}(Q)$, and instabilities should result also in this case. In fact, this intuitive argument is confirmed by the more sophisticated analysis described below (some technical details are carried out in the Appendix), which shows that also the incommensurate SWs are unstable for $\delta$ close to $\delta_{0}(Q)$, but when $|Q|>\pi / 2$ only through higher order instabilities, which are thus more difficult to detect numerically or experimentally.

To prove the instability of analytic SWs we use here the method of 'band analysis' [4]. 8 Its basic idea is to embed the non-Hermitian eigenvalue problem

\footnotetext{
8 The same approach can be used for small-amplitude commensurate SWs, see
} 
(33) into a wider eigenvalue problem but for a Hermitian operator, which has more properties. Then, according to [4], we associate this problem with an extended eigenvalue problem

$$
i \dot{\epsilon}_{n}-\delta \epsilon_{n}-2\left|\psi_{n}\right|^{2} \epsilon_{n}-\psi_{n}^{2} \epsilon_{n}^{*}-C\left(\epsilon_{n+1}+\epsilon_{n-1}-2 \epsilon_{n}\right)=E \epsilon_{n} .
$$

The eigenvalues and the corresponding eigensolutions of this eigenequation can be searched again with the form (31), where $\omega$ is a real parameter. Then, the eigenvalues $E$ with eigenvectors $\left\{a_{n}, b_{n}\right\}$ are those of a self-adjoint operator:

$$
\begin{aligned}
& \left(-\omega+2 C-\delta-2 \psi_{n}^{2}\right) a_{n}-C\left(a_{n+1}+a_{n-1}\right)-\psi_{n}^{2} b_{n}=E a_{n} \\
& -\psi_{n}^{2} a_{n}-C\left(b_{n+1}+b_{n-1}\right)+\left(\omega+2 C-\delta-2 \psi_{n}^{2}\right) b_{n}=E b_{n} .
\end{aligned}
$$

Writing as before $U_{n}=a_{n}+b_{n}$ and $W_{n}=a_{n}-b_{n},(37)$ becomes

$$
\begin{aligned}
\left(2 C-\delta-3 \psi_{n}^{2}\right) U_{n}-C\left(U_{n+1}+U_{n-1}\right)-\omega W_{n} & =E U_{n} \\
-\omega U_{n}+\left(2 C-\delta-\psi_{n}^{2}\right) W_{n}-C\left(W_{n+1}+W_{n-1}\right) & =E W_{n},
\end{aligned}
$$

or

$$
\left(\begin{array}{cc}
\mathcal{L}_{1} & -\omega \\
-\omega & \mathcal{L}_{0}
\end{array}\right)\left(\begin{array}{c}
\left\{U_{n}\right\} \\
\left\{W_{n}\right\}
\end{array}\right)=E\left(\begin{array}{c}
\left\{U_{n}\right\} \\
\left\{W_{n}\right\}
\end{array}\right)
$$

with $\mathcal{L}_{0}$ and $\mathcal{L}_{1}$ defined by Eqs. (33). Then, each eigenvalue $E_{\nu}(\omega)$ of the problem (39) becomes a smooth, real function of $\omega$ called band (symmetric with respect to $\omega=0$ ), which also depends smoothly on the other model parameters (as before, we only need to consider variations of the parameter $\delta^{\prime}=\frac{\delta}{C}$ ). If we assume that the parameter $\omega$ represents the eigenfrequencies for the initial non-Hermitian problem (33), the problem (39) reduces to the problem (33) for the particular value $E=0$. Thus the real eigenfrequencies $\omega_{s}$ of (33) are determined by the intersections $E_{\nu}\left(\omega_{s}\right)=0$ of the bands with the axis $E=0$, and their Krein signature by $\operatorname{sign}\left(-\frac{d E_{\nu}}{d \omega}\right)$ at $\omega=\omega_{s}>0$ [4]. Varying the model parameter $\delta^{\prime}$ the bands will generally move in the $(\omega, E)$ plane, and if a band loses a pair of intersections with the zero-energy axis (i.e., if a 'gap' opens around this axis) an instability occurs for the initial problem (33).

Appendix.

${ }^{9}$ For an oscillatory (Krein) instability, two symmetric pairs of intersection are simultaneously lost. 
At the linear limit $\left(\psi_{n} \rightarrow 0\right.$ and $\left.\delta \rightarrow \delta_{0}(Q)\right)$, the eigenvalue problem (39) is easily solved by introducing a wave vector $q$ and writing

$$
U(q)=\sum_{n} U_{n} \mathrm{e}^{\mathrm{i} q n}, \quad W(q)=\sum_{n} W_{n} \mathrm{e}^{\mathrm{i} q n} .
$$

At this limit, there are two sets of plane wave solutions $U_{n}= \pm W_{n}=\mathrm{e}^{\mathrm{i} q n}$ with corresponding eigenvalues

$$
E_{0 \pm}(q ; \omega)=2 C-\delta_{0}(Q)-2 C \cos q \mp \omega=2 C(\cos Q-\cos q) \mp \omega .
$$

For a finite system with $N$ sites, $q=2 \pi k / N$ with $0 \leq k<N$ integer, and thus there are $2 N$ bands described by straight lines with slope $\mp 1$ and indexed by the wave vector $q$ (Fig. $8(\mathrm{a})$ ). Each band $E_{0 \pm}(q ; \omega)$ intersects $E=0$ at $\omega_{ \pm}(q)= \pm\left(2 C-\delta_{0}(Q)-2 C \cos q\right) \equiv \omega_{s}(q)$, so that we recover the result (35) that Eq. (33) has $2 N$ eigenvalues on the real axis so that the solution $\psi_{n} \equiv 0$ is linearly stable as expected, and remains stable also in the limit of large $N$.

When $\psi_{n}$ is small but non-zero, it can be treated as a perturbation for the operator in the left-hand side of Eq. (39). Then, gaps will generally open in the continuous band spectrum of this operator at points or lines of degeneracy. Such a gap might suppress a band intersection $E_{0 \pm}(\omega)=0$ with the real axis, and thus generate a linear instability. There are two kinds of degeneracies. The first kind occurs for both branches of eigenenergies $E_{0+}(q ; \omega)$ and $E_{0-}(q ; \omega)$ for all $\omega$, since $\left.E_{0 \pm}(q ; \omega)=E_{0 \pm}(2 \pi-q ; \omega)\right)$. The second kind occurs at transverse intersection points, where bands with opposite slope intersect if $E_{0-}(q ; \omega)=$ $E_{0+}\left(q^{\prime} ; \omega\right)$ for some $q, q^{\prime}$.

For an analytic SW, the hull function (c.f. Eq. (27)) $\chi_{S}^{2}(x)$ of $\psi_{n}^{2}$ can be chosen even and $\pi$-periodic, and thus $\psi_{n}^{2}$ can be expanded in a Fourier series as

$$
\psi_{n}^{2}=\sum_{p} f_{p} \mathrm{e}^{\mathrm{i} 2 p(n Q+\phi)},
$$

where $f_{p}=f_{-p}$. We can choose the arbitrary phase as $\phi=0$. Since, as for conventional perturbation theory, the total sum of the gap openings will be proportional to $\sum_{p}\left|f_{p}\right|$, perturbation theory will be consistent in principle only when this sum is convergent and small. Thus, perturbation theory is applicable for incommensurate SWs only when $\chi_{S}(x)$ is analytic, in which case $f_{p}$ decays exponentially for $p \rightarrow \infty$, and not when $\psi_{n}$ is represented by cantori because then this sum is divergent. Then, considering only the regime close to the linear limit where the SW is analytic with small amplitude $\epsilon_{s}, \chi_{S}(x)$ becomes close to a harmonic function $\epsilon_{s} \cos x$, and we have

$$
f_{0} \approx 2 f_{1}=2 f_{-1} \approx \epsilon_{s}^{2} / 2>0
$$


while the other Fourier coefficients $f_{p}$ will be small of order $\epsilon_{s}^{2|p|}$. Using the space Fourier transform (40), Eq. (38) becomes without approximations

$$
\begin{aligned}
& \left(2 C-\delta-2 C \cos q-3 f_{0}\right) U(q)-3 \sum_{p \neq 0} f_{p} U(q+2 p Q)-\omega W(q)=E U(q) \\
& -\omega U(q)+\left(2 C-\delta-2 C \cos q-f_{0}\right) W(q)-\sum_{p \neq 0} f_{p} W(q+2 p Q)=E W(q) .
\end{aligned}
$$

Thus, this equation couples the space of solutions $(U(q), W(q))$ with wave vector $q$ of the unperturbed problem (39) with those with wave vector $q+2 p Q$, and the coupling strength is proportional to $f_{p}$. The matrix of the operator corresponding to the left-hand side of Eq. (44) can be decomposed into blocks of $2 \times 2$-matrices, which we denote as $\mathbf{D}(q)$ for the diagonal part acting on the subspace with wave vector $q$, and $-f_{p} \mathbf{A}$ for the parts which couple subspace $q$ with subspace $q+2 p Q$ :

$$
\mathbf{D}(q)=\left(\begin{array}{cc}
2 C-\delta-2 C \cos q-3 f_{0} & -\omega \\
-\omega & 2 C-\delta-2 C \cos q-f_{0}
\end{array}\right) ; \mathbf{A}=\left(\begin{array}{ll}
3 & 0 \\
0 & 1
\end{array}\right) .
$$

The eigenvalues of the diagonal part $\mathbf{D}(q)$ are given by

$$
E_{ \pm}(q ; \omega)=2 C-\delta-2 C \cos q-2 f_{0} \mp \sqrt{\omega^{2}+f_{0}^{2}},
$$

which determines the downward shift of the unperturbed eigenvalues $E_{0 \pm}(q ; \omega)$ (cf. Eq. (41)) caused by the static term $f_{0}$ in the expansion (42) (which has the same order of magnitude as $f_{1}$ ). The perturbation due to $\left|f_{p}\right|,|p| \geq 1$, will then act mostly by raising the degeneracies of these eigenvalues. The first kind of degeneracy,

$$
E_{+}(q ; \omega)=E_{+}(2 \pi-q ; \omega) \text { and } E_{-}(q ; \omega)=E_{-}(2 \pi-q ; \omega)
$$

is raised when $q+2 p Q=-q(\bmod 2 \pi)$, or

$$
q=-p Q(\bmod \pi)
$$

with $p$ integer. Then, a gap of width proportional to $\left|f_{p}\right|$ appears between the two curves $E_{+}(-p Q ; \omega)$ and $E_{+}(p Q ; \omega)$, and an identical gap opens between the curves $E_{-}(-p Q ; \omega)$ and $E_{-}(p Q ; \omega)$ (Fig. $8(\mathrm{~b})$ ). However, the existence of these gaps does not change the number of intersections $E_{ \pm}(q ; \omega)=0$ which exist in the linear limit, and thus does not induce any instabilities for the SWs. 


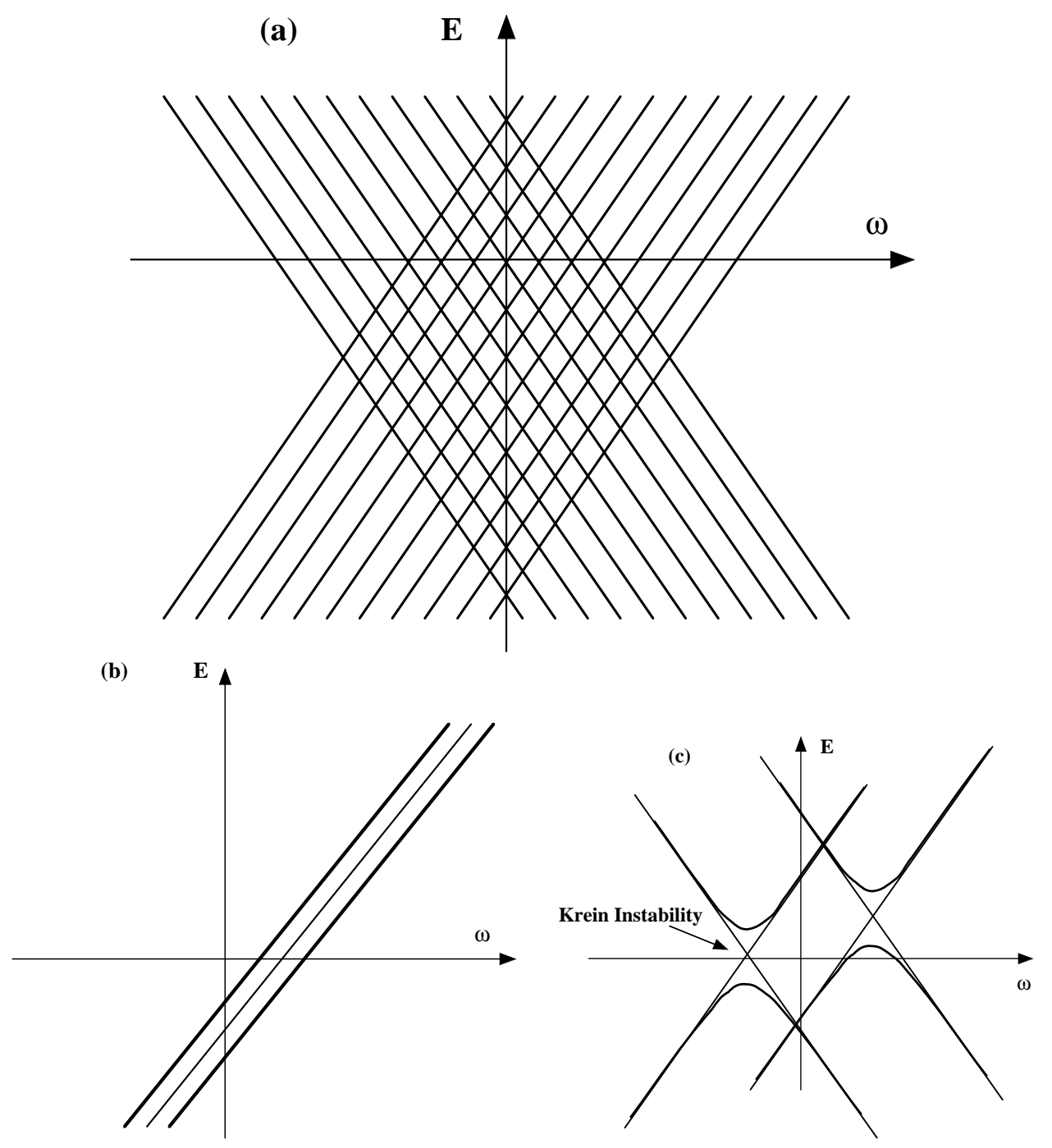

Fig. 8. (a) Schematical picture of the unperturbed band structure $E(\omega)=E_{0 \pm}(q ; \omega)$ (Eq. 41); (b) Gap opening for the first kind of degeneracy (46) (no instability); (c) Gap opening for the second kind of degeneracy (48). An instability is generated if the gap overlaps the axis $E=0$.

For the second kind of degeneracy, at transverse intersection points, a gap with width proportional to $\left|f_{p}\right|$ opens if

$$
E_{+}\left(q ; \omega_{p}\right)=E_{-}\left(q+2 p Q ; \omega_{p}\right) \equiv E_{p}\left(\omega_{p}\right)
$$

and a straightforward calculation yields the perturbed eigenvalues at $\omega_{p}$ to 
order $f_{p}: 10$

$$
E_{p \pm}^{\prime}\left(\omega_{p}\right)=E_{p}\left(\omega_{p}\right) \pm \frac{\left|f_{p} \omega_{p}\right|}{\sqrt{\omega_{p}^{2}+f_{0}^{2}}}
$$

If $E=0$ belongs to one of these gaps, the SW becomes unstable (see Fig. 8 (c)). These intersections occur when

$$
\begin{aligned}
\frac{1}{C} \sqrt{\omega_{p}^{2}+f_{0}^{2}} & =\cos (q+2 p Q)-\cos q \\
E_{p} & =2 C-\delta-2 f_{0}-C \cos (q+2 p Q)-C \cos q
\end{aligned}
$$

It comes out readily from Eqs. (50) and (51) that for a given $p$, these intersection points are located on an ellipse in the $(\omega, E)$-plane given by

$$
\frac{\omega_{p}^{2}}{4 C^{2} \sin ^{2} p Q-f_{0}^{2}}+\frac{\left(2 C-\delta-2 f_{0}-E_{p}\right)^{2}}{4 C^{2} \cos ^{2} p Q-f_{0}^{2} \cot ^{2} p Q}=1 \text {, }
$$

which exists for $\left|f_{0}\right|<2 C|\sin p Q|$, with its center at $\omega=0$ and $E=2 C-$ $\delta-2 f_{0}$. Thus, this ellipse represents the locus of the middle of the gaps which are opened by the perturbation of order $f_{p}$, and an instability of the SW is generated when this ellipse intersects the axis $E=0$, that is when

$$
\left(2 C-\delta-2 f_{0}\right)^{2}<4 C^{2} \cos ^{2} p Q-f_{0}^{2} \cot ^{2} p Q
$$

The existence of these ellipses of gaps is confirmed by numerical calculations of the set of bands $E(\omega)$ (see Fig. 9). In the incommensurate case, $|p|$ may run from 1 to $+\infty$, and thus there are infinitely many ellipses which cover densely the rhombic area where the set of curves $E_{-}(q ; \omega)$ intersects the set of curves $E_{+}\left(q^{\prime} ; \omega\right)$ for arbitrary $q$ and $q^{\prime}$. When $E=0$ crosses this area, there are surely infinitely many ellipses intersecting this axis, but the instabilities which are generated at these intersections become very weak and numerically invisible when $p$ becomes large.

As $p=1$ corresponds to the ellipse with the largest gaps, it yields the strongest instabilities if these gaps are opening around $E=0$. For $p=1$ and in the limit of zero amplitude SWs $\left(f_{0}=0, \delta=\delta_{0}(Q)\right)$, the particular ellipse (52) for

\footnotetext{
$\overline{{ }^{10} \text { Here, }}$ the possible interference with resonances of the first kind (47) has been neglected. The case of simultaneous resonances, which occurs at special points, is discussed in the Appendix.
} 

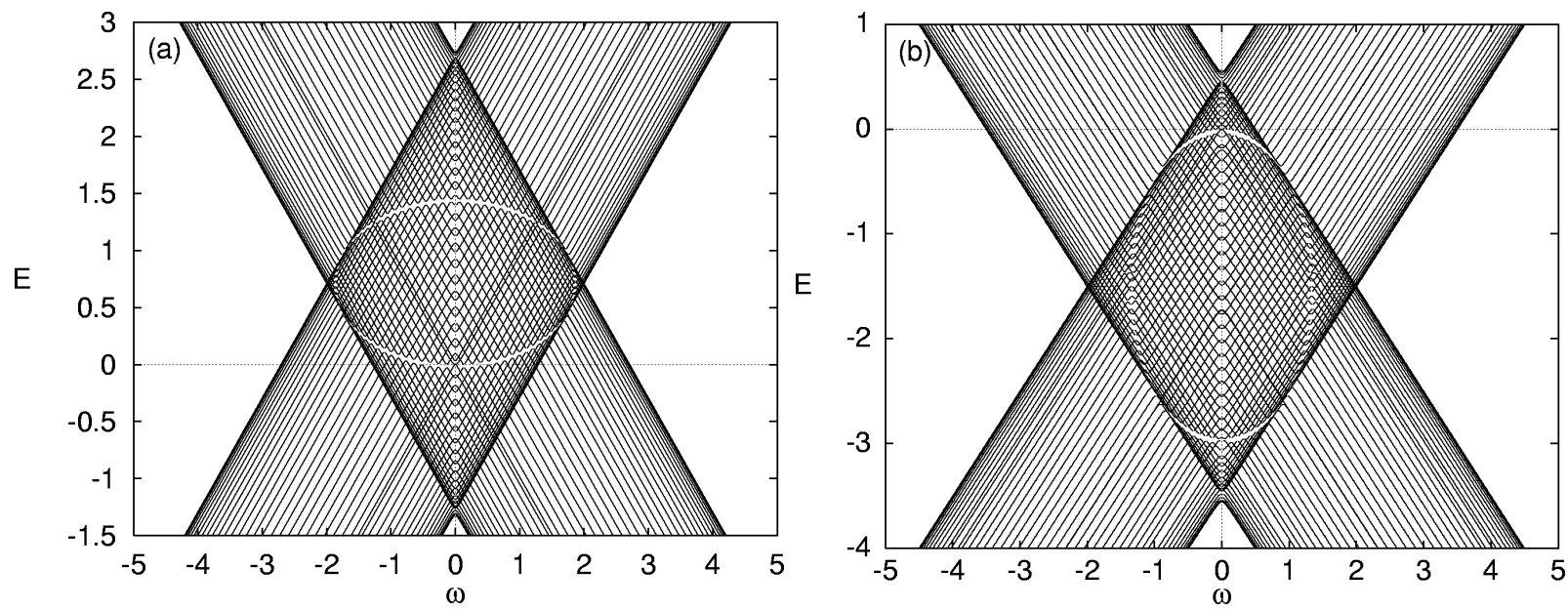

Fig. 9. Band spectrum of (39) for a SW with (a) $Q / 2 \pi=17 / 89 \simeq \sigma_{G} / 2(Q<\pi / 2)$, $\delta^{\prime}=1.23$, and (b) $Q / 2 \pi=34 / 89 \simeq \sigma_{G}(Q>\pi / 2), \delta^{\prime}=3.4(C=1)$. The visible gap openings occur along the ellipse (52) with $p=1$.

the intersections $\left(\omega_{1}, E_{1}\right)$ has the equation:

$$
\frac{\omega_{1}^{2}}{4 C^{2} \sin ^{2} Q}+\frac{\left(2 C \cos Q-E_{1}\right)^{2}}{4 C^{2} \cos ^{2} Q}=1 .
$$

Thus, it is tangent to the axis $E=0$ at $\omega=0$, and it lies above $E=0$ for wave vectors $0<Q<\pi / 2$ and below for $\pi / 2<Q<\pi$. When the SW amplitude $\epsilon_{s}$ is small but nonzero, we obtain using Eqs. (26) and (43) that, to lowest order in $\epsilon_{s}$, the 'midgap' ellipse (52) with $p=1$ cuts the axis $\omega=0$ at $E=-\epsilon_{s}^{2} / 4$. Then, using Eq. (49) to calculate the perturbed eigenvalues for small but nonzero $\left|\omega_{1}\right|$ and $\left|f_{0}\right| \ll\left|\omega_{1}\right|$ we obtain, again by using (43), that $E_{1-}^{\prime} \approx-\epsilon_{s}^{2} / 2$ and $E_{1+}^{\prime} \approx 0$ to order $\epsilon_{s}^{2}$ when $\left|\omega_{1}\right|$ is small but nonzero. Thus, we should expect that when $0<Q<\pi / 2$ (so that the unperturbed ellipse is above the axis $E=0$ ), gaps opening in the lower part of the ellipse will surround the axis $E=0$ for some interval of $|\omega|$ close to $\omega=0 . \square$ As a consequence, $S W s$ with $0<Q<\pi / 2$ become unstable through firstorder oscillatory instabilities for arbitrarily small amplitude $\epsilon_{s}$ (Fig. 9 (a)). By contrast, as for $\pi / 2<Q<\pi$ the unperturbed ellipse lies below the axis $E=0$, we expect all first-order gaps outside the immediate neighbourhood of $\omega=0$ to open strictly below $E=0$. Moreover, as for analytic SWs there are two explicit eigensolutions with $E=0$ at $\omega=0$ (the phase mode and the sliding mode, see Appendix A.2), there is no gap around $E=0$ (and consequently no instability) for $\omega=0$. We also confirmed by numerical calculations (Figs. 9 (b) and 10 (a)) that no first-order gaps open around $E=0$, so that for SWs with $\pi / 2<Q<\pi$ no first-order instabilities develop. However, it is

${ }^{11}$ A similar situation with first order instability occurs also for all commensurate SWs with $Q \neq \pi$, see Appendix. 
important to remark that the above estimate based on Eq. (49) is not valid in the immediate neighbourhood of $\omega=0$. Actually, in the zero-amplitude limit $\epsilon_{s}=0$, the point $E_{1}=0, \omega_{1}=0$ where the unperturbed first-order ellipse (54) is tangent to the axis $E=0$ is such that $E_{0+}(0)=E_{0-}(0)=0$, which implies by Eqs. (41) that $q= \pm Q$ so that also the first resonance condition (47) is fulfilled for $|p|=1$. Thus, close to the origin $E=0, \omega=0$ the two different kinds of gap openings strongly interfere with each other at the same order $(p=1)$, and thus must be considered simultaneously as described in the Appendix.
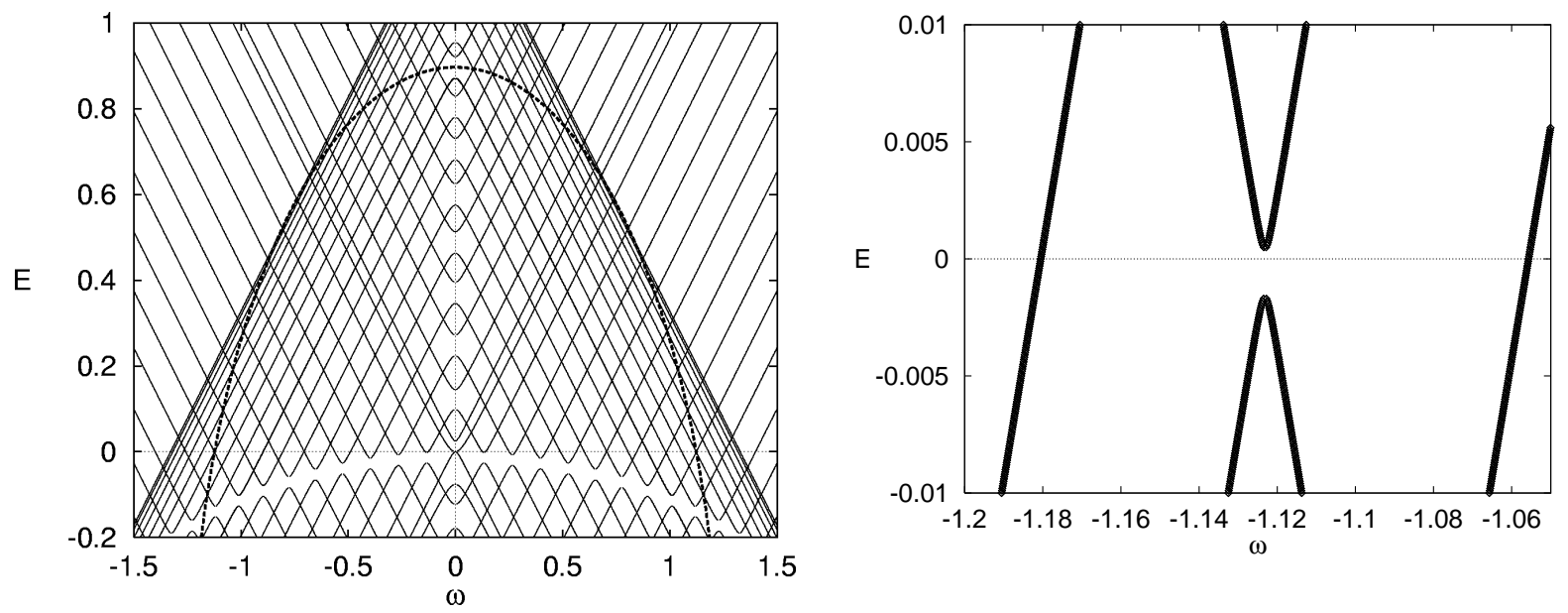

Fig. 10. Part of the band spectrum of (39) for a SW with $Q / 2 \pi=27 / 89(Q>\pi / 2)$ and $\delta^{\prime}=2.62$. The thick dashed line in the left figure is the second-order ellipse $(p=2$ in Eq. (52)). Right figure shows a magnification of the part around $E=0$ where a second-order gap opens.

Thus, when $\pi / 2<Q<\pi$, the strongest instability appears for the smallest $p>1$ such that the condition (53), which in the zero-amplitude limit reduces to $|\cos p Q|>|\cos Q|$, is fulfilled. An example with $p=2$ is illustrated in Fig. 10. Then, there always exists a $p$ such that, for arbitrarily small amplitude, the $S W$ is unstable to order $p$. Anyway, since the width of the gap openings, and therefore also the maximum instability growth rates, are proportional to $\left|f_{p}\right|$, the instabilities become very weak for $Q$ close to $\pi$ as the smallest $p$ yielding an instability becomes large, and they could be practically invisible in numerical simulations for finite-size systems. We might therefore describe these analytic SWs as 'quasi-stable'.

\section{Numerical analysis of SWs in KG chains}

Let us now return to the original KG model (5), for which we will investigate numerically the validity of our previous results when the conditions of small amplitude and small coupling are not necessarily fulfilled. In general, when 
the SW amplitude is non-negligible also harmonics $a_{n}^{(p)}$ with $|p| \neq 1$ in the Fourier expansion (10) will affect the dynamics. Moreover, as already mentioned in Sec. 2.2 in connection with Eqs. (15)-(18), when the coupling $C_{K}$ is increased also the small-amplitude dynamics will in general be affected as the long-range nonlinear interaction terms in the extended DNLS equation become non-negligible, and in particular resonances may occur also for smallamplitude SWs if higher harmonics of the solution enter the linear phonon band. We will consider the particular cases of the soft Morse potential (3) and the hard quartic potential (4). As described in Sec. 3.2, the SWs are obtained by numerical continuation of the multibreathers with coding sequences defined by Eqs. (28)-(29) (modified according to footnote 5 in the case of a hard potential), using a modified Newton scheme (described in detail in Ref. [11]) to find numerically exact time-reversible solutions $\left\{u_{n}(t)\right\}$ with a given time period $T_{b}=2 \pi / \omega_{b}$. The linear stability of these solutions is then determined by numerical integration of the Hill equations (6) over one period $T_{b}$, defining a linear symplectic $2 N$-dimensional map given by the Floquet matrix $\mathbf{T}_{\mathbf{0}}\left(\left\{u_{n}\right\}\right)$ :

$$
\left(\begin{array}{c}
\left\{\epsilon_{n}\left(T_{b}\right)\right\} \\
\left\{\dot{\epsilon}_{n}\left(T_{b}\right)\right\}
\end{array}\right)=\mathbf{T}_{\mathbf{0}}\left(\begin{array}{c}
\left\{\epsilon_{n}(0)\right\} \\
\left\{\dot{\epsilon}_{n}(0)\right\}
\end{array}\right)
$$

As $\mathbf{T}_{\mathbf{0}}$ is symplectic, for each eigenvalue $\Lambda$ there are also eigenvalues at $\Lambda^{*}$ and $1 / \Lambda$. Then, the solution $\left\{u_{n}(t)\right\}$ is linearly stable if and only if all the eigenvalues of $\mathbf{T}_{\mathbf{0}}\left(\left\{u_{n}\right\}\right)$ lie on the unit circle. The Krein signature associated to a complex conjugated pair of eigenvalues $\Lambda=r \mathrm{e}^{ \pm \mathrm{i} \theta}$ is defined as $[32,4]$

$$
\mathcal{K}(\theta)=\operatorname{sign}\left(\mathrm{i} \sum_{n}\left[\epsilon_{n} \dot{\epsilon}_{n}^{*}-\epsilon_{n}^{*} \dot{\epsilon}_{n}\right]\right),
$$

where $\left\{\epsilon_{n}, \dot{\epsilon}_{n}\right\}$ is the eigenvector of $\mathbf{T}_{\mathbf{0}}$ with eigenvalue $r \mathrm{e}^{+\mathrm{i} \theta}$. As before, instabilities can only occur through collisions of eigenvalues with opposite Krein signature.

At the anticontinuous limit $C_{K}=0$, each site with code $\left|\tilde{\sigma}_{n}\right|=1$ yields a degenerate pair of eigenvalues at +1 , while each site with code $\tilde{\sigma}_{n}=0$ yields a complex conjugated pair of eigenvalues at $\mathrm{e}^{ \pm \mathrm{i} 2 \pi / \omega_{b}}$. When $C_{K}$ is increased from zero, the degeneracies are raised and the spreading of the eigenvalues is just analogous to the DNLS case discussed in the previous section. For type $H$ SWs all eigenvalues will initially remain on the unit circle (constituting bands for commensurate SWs and Cantor-spectra for incommensurate SWs as for the DNLS case), preserving the linear stability until the first oscillatory instability appears through a collision on the unit circle between eigenvalues of different Krein signature originating from +1 and $\mathrm{e}^{ \pm \mathrm{i} 2 \pi / \omega_{b}}$, respectively. 
For type $E$ SWs, in addition pairs of eigenvalues originating from +1 will immediately go out on the real axis when $C_{K}$ becomes nonzero. Thus, in general, the instability scenario we observe is as expected identical to that of the DNLS model, as long as the amplitude and coupling remain small, and no higher-order resonances occur. The scenario outside this regime is however quite different for the hard quartic and the soft Morse potentials, and we will therefore discuss them separately below.

\subsection{The quartic potential}

Let us first discuss SWs with wave vector $Q$ in the hard quartic potential (4), having frequencies $\omega_{b}>\omega_{l}(Q)>1$ (c.f. Eq. (8) and Fig. 5). This case is particularly simple since the on-site potential is spatially symmetric, $V(u)=$ $V(-u)$, and consequently the time-periodic solutions with fundamental period $T_{b}=2 \pi / \omega_{b}$ fulfill $u_{n}\left(t+T_{b} / 2\right)=-u_{n}(t)$ (i.e., the time-reversible solutions are also time antisymmetric with respect to $t=T_{b} / 4$ ), so that we can restrict our numerical Newton algorithm to search for solutions fulfilling this condition. Note that this condition is not fulfilled for any even higher harmonic of the solution (i.e., with period $T_{b} / p, p$ even), so that there can be no resonances with even harmonics disturbing the unique continuation of the SW. Actually, we already remarked in Sec. 2.2 that there is no coupling between even and odd harmonics of the solutions for the quartic potential. Moreover, as $V^{\prime \prime}\left(u_{n}\right)$ in the Hill equations (6) in this case has the fundamental period $T_{b} / 2$ rather than $T_{b}$, it is convenient to redefine the Floquet matrix from (55) as

$$
\left(\begin{array}{c}
\left\{-\epsilon_{n}\left(T_{b} / 2\right)\right\} \\
\left\{-\dot{\epsilon}_{n}\left(T_{b} / 2\right)\right\}
\end{array}\right)=\mathbf{T}_{\mathbf{0}}^{\prime}\left(\begin{array}{c}
\left\{\epsilon_{n}(0)\right\} \\
\left\{\dot{\epsilon}_{n}(0)\right\}
\end{array}\right) .
$$

Then, the eigenvalues of $\mathbf{T}_{\mathbf{0}}^{\prime}$ corresponding to sites with code $\tilde{\sigma}_{n}=0$ will at the anticontinuous limit be located at $\mathrm{e}^{ \pm \mathrm{i} \pi / \omega_{b}}$, i.e., at half the angle of the corresponding eigenvalues of $\mathbf{T}_{\mathbf{0}}$.

Considering now small-amplitude solutions with increasing coupling $C_{K}$, we find for different wave vectors $Q$ three qualitatively different scenarios, depending on whether the second and/or third harmonic of the SW frequency $\omega_{b}$ enters the linear phonon band. In general, the condition that the $p$ th harmonic of a SW with wave vector $Q$ has entered the phonon band can be written as

$$
\sqrt{1+4 C_{K} \sin ^{2} \frac{Q}{2}}<\omega_{b}<\frac{1}{p} \sqrt{1+4 C_{K}}
$$



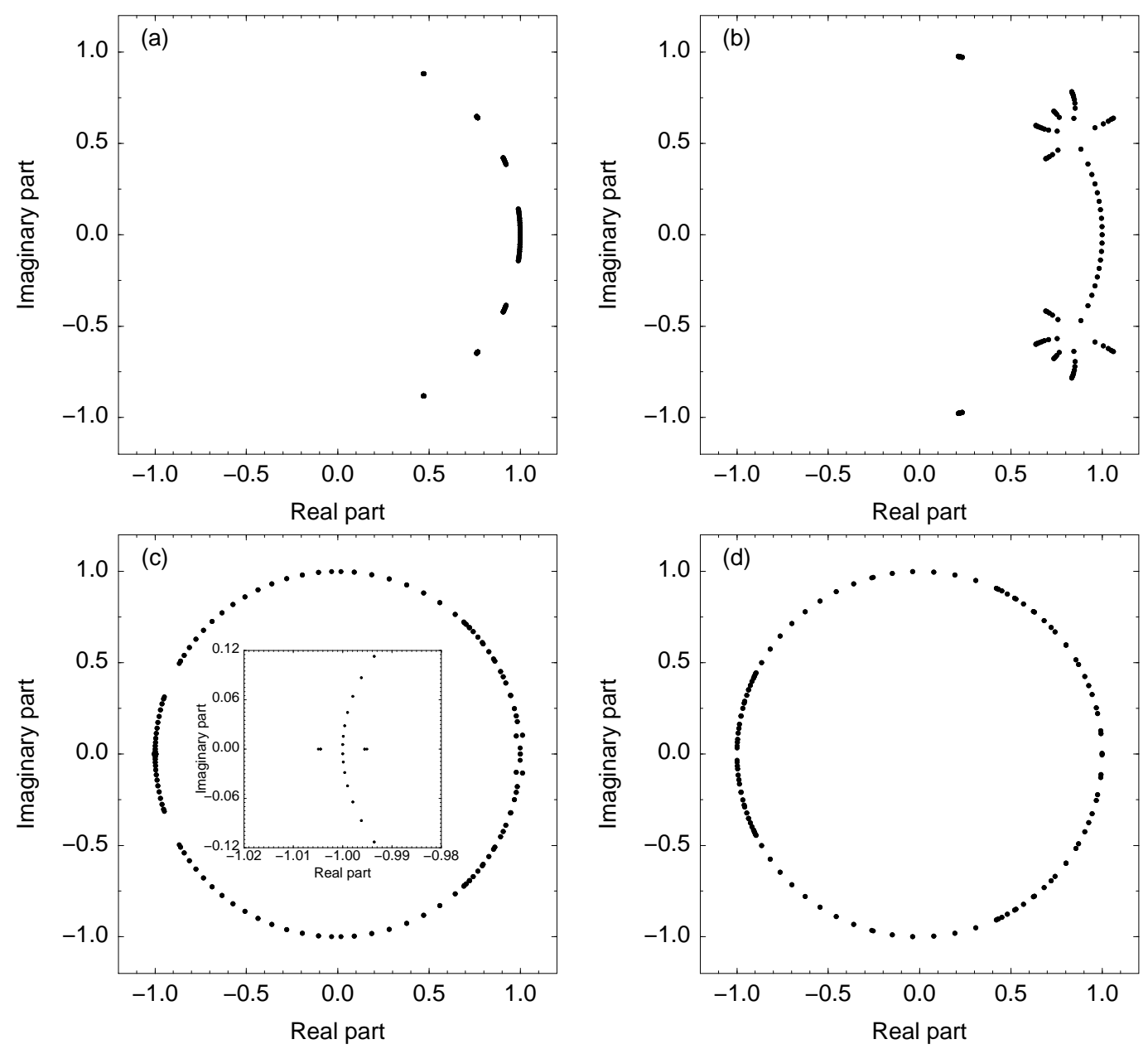

Fig. 11. Eigenvalues of the Floquet matrix $\mathbf{T}_{\mathbf{0}}^{\prime}$ for a type $H \mathrm{SW}$ with wave vector $Q=\pi / 4$ in a finite-size $\mathrm{KG}$ lattice $(N=120)$ with the quartic potential (4), $\omega_{b}=1.8$, and increasing $C_{K}$ : (a) $C_{K}=0.199$ (stable wave); (b) $C_{K}=0.699$ (oscillatory instabilities); (c) $C_{K}=2.773$ (additional non-oscillatory instabilities due to collisions at -1 resulting from second-harmonic resonances, see inset); and (d) $C_{K}=3.399$ (return to stability close to the linear limit for finite-size system).

This condition is fulfilled only when

$$
\sin \frac{Q}{2}<\frac{1}{p} \quad \text { and } \quad C_{K}>\frac{p^{2}-1}{4\left(1-p^{2} \sin ^{2} \frac{Q}{2}\right)}
$$

Thus, for SWs with wave vector $Q>\pi / 3$ there are no higher order resonances. In this case, the SWs can for fixed frequency $\omega_{b}$ be continued versus $C_{K}$ until they reach their linear limit at $C_{K}=\frac{\omega_{b}^{2}-1}{4 \sin ^{2} \frac{Q}{2}}\left(\right.$ where $\omega_{b}=\omega_{l}(Q)$ according to (8)), and the instability scenario during this continuation is completely analogous to that observed in the DNLS approximation discussed in the previous section, also when $\omega_{b}$ is not close to 1 . On the other hand, when $Q<\pi / 3$ the second harmonic enters the phonon band before the SW reaches its linear 
limit if $\omega_{b}$ is large enough. As discussed above, this resonance does not affect the unique continuation of the SW which still can be continued to its linear limit, but causes additional non-oscillatory instabilities which are signalled by collisions of eigenvalues of $\mathbf{T}_{\mathbf{0}}^{\prime}$ at -1 . An example of the instability scenario observed in this regime is illustrated in Fig. 11.

The third regime occurs when $\sin \frac{Q}{2}<\frac{1}{3}$, or $Q \lesssim 0.216 \pi$. Then, if $\omega_{b}$ is large enough so that the conditions (58)-(59) are fulfilled for $p=3$, the $\mathrm{SW}$ with frequency $\omega_{b}$ will generally bifurcate with solutions with frequency $3 \omega_{b}$ before reaching the linear limit. These bifurcations are signalled by collisions at +1 in the Floquet eigenvalue spectrum of $\mathbf{T}_{\mathbf{0}}^{\prime}$. We will here not attempt to give any rigorous description of the possible nature of these (sometimes rather complicated) bifurcations, but rather make some qualitative general observations (more details are given in [35]). A typical example of a scenario resulting from such bifurcations is illustrated in Fig. 12. In this case, the continuation from the anticontinuous limit of the SW with wave vector $Q=4 \pi / 21$ and $\omega_{b}=4.5$ yields the first collision at +1 resulting from a third-harmonic resonance at $C_{K} \approx 44.18$ (see Fig. $12(\mathrm{c})$ and the magnification in (d)). This is slightly before the third harmonic enters the linear phonon band (Eq. (58) yields $\left.C_{K}=45.3125\right)$, since the wave amplitude is non-negligible and the resonating wave is a nonlinear rather than a linear SW. As illustrated in Fig. 12 (a) and (d), it is possible to continue smoothly the solution through this bifurcation, but the nature of the solution changes drastically. In fact, this scenario typically occurs in two steps: at the first bifurcation point the solution changes into an intermediate (unstable) solution, and later, at a second bifurcation point $\left(C_{K} \approx 44.8\right.$ in Fig. 12) into the final solution. The latter is the SW whose wave vector $Q^{\prime}$ (close to $\pi$ ) coincides with the wave vector of the resonating eigenmode ${ }^{12}$, and it corresponds to an anticontinuous coding sequence generated by Eqs. (28)-(29) but with the coding sequence $\tilde{\sigma}_{n}$ taking the values \pm 3 instead of \pm 1 . Then, the smooth continuation versus $C_{K}$ for fixed $\omega_{b}$ follows this new solution without further bifurcations, until it reaches its linear limit at $C_{K}=\frac{9 \omega_{b}^{2}-1}{4 \sin ^{2} \frac{Q^{\prime}}{2}}$.

However, it is important to note that there generally exists also a solution with the original wave vector $Q$ close to the linear limit (in smooth continuation of the linear SW with wave vector $Q$ ), but due to the third-harmonic resonances there should in this case be no continuous path in parameter space connecting this linear SW with the SW with wave vector $Q$ generated from the anticontinuous limit. However, if the numerical continuation is performed with less care, the resonances might be missed and an apparently (but falsely!) smooth continuation of the SW with wave vector $Q$ from the linear to the anticontinuous limit can be observed as illustrated in Fig. 12 (b)-(c). The more

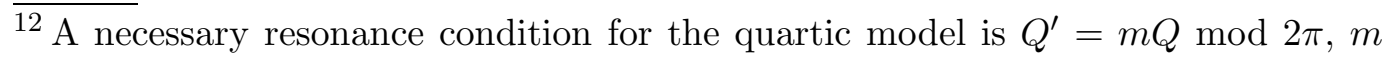
odd. The strength of the resonance generally decreases with $|m|$. 
(a)
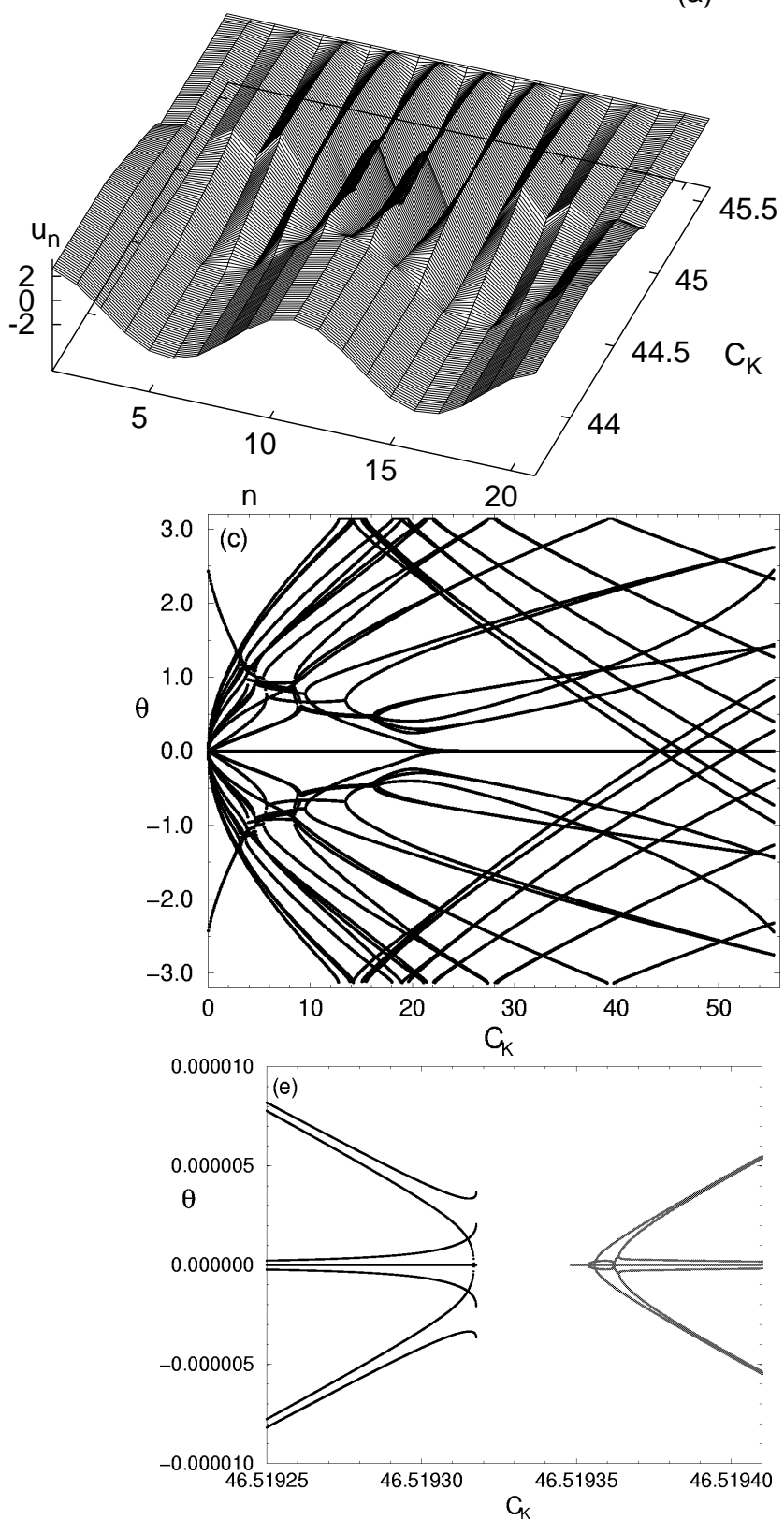

45.5
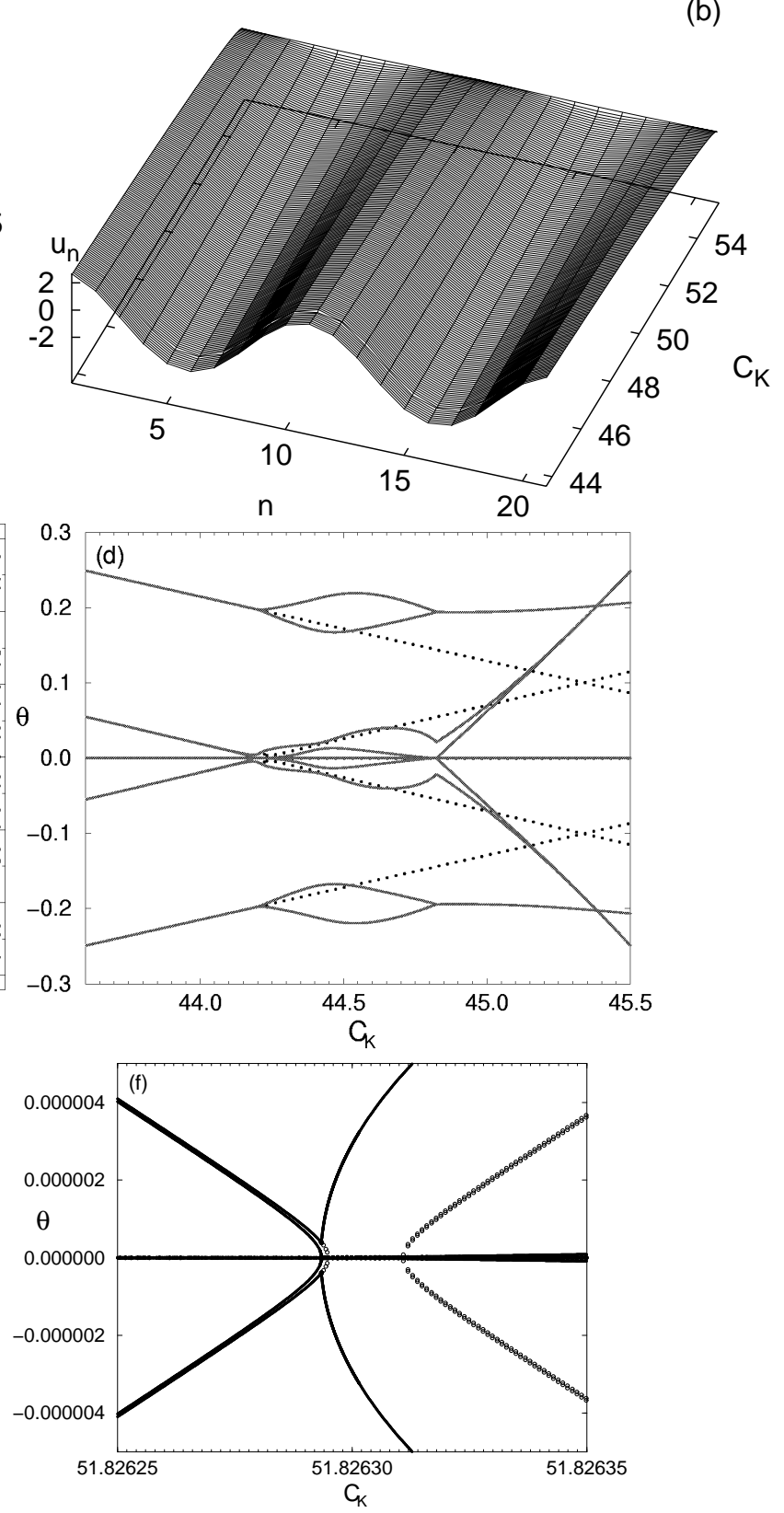

Fig. 12. Bifurcation scenario resulting from third-harmonic resonances in the quartic model. The initial type $H$ SW has wave vector $Q=4 \pi / 21$ and frequency $\omega_{b}=4.5$. (a) Smooth continuation for increasing $C_{K}$ of a SW with wave vector $Q$ generated from the anticontinuous limit into a type $H$ SW with frequency $3 \omega_{b}$ and wave vector $Q^{\prime}=20 \pi / 21$. (b) Intentionally 'careless' continuation from the linear limit for decreasing $C_{K}$ of a SW with wave vector $Q$, where the step size in $C_{K}$ has been chosen large enough to jump over all resonance regimes (note the small visible gap at $C_{K} \approx 44.2$ corresponding to the resonance in (a)). (c) Arguments of all eigenvalues $r \mathrm{e}^{\mathrm{i} \theta}$ of the Floquet matrix $\mathbf{T}_{\mathbf{0}}^{\prime}$ versus coupling for one unit cell $(N=21)$ of the SW with wave vector $Q$ in (b). (d) Magnification of the first resonance in (c). The solid line represents the smooth continuation from below as in (a), the dotted line the continuation from above as in (b) which stops at $C_{K} \approx 44.22$. (e), (f) Magnification of the second and third resonances, respectively (see text for explanation). 
careful numerics yields the behaviour close to the three resonances illustrated in Fig. 12 (d)-(f). Thus, we find that there exists a SW with the original wave vector $Q$ also immediately after the first resonance, but attempts to continue it towards smaller $C_{K}$ (dotted line in Fig. $12(\mathrm{~d})$ ) fail for $C_{K} \approx 44.22$. Similarly, the attempts to continue it towards larger $C_{K}$ fail when approaching the second resonance at $C_{K} \approx 46.51932$ (left part of Fig. 12 (e)) [3], so that its existence region apparently is limited by these two resonances. A similar behaviour is observed also between the second and the third resonances. Approaching the second resonance from above the continuation of the SW with wave vector $Q$ stops at $C_{K} \approx 46.51935$ (right part of Fig. 12 (e)), while approaching the third resonance from below it transforms, via an intermediate solution appearing through a collision at +1 at $C_{K} \approx 51.826293472$ (solid line in Fig. 12 (f)), into a SW with wave vector $Q^{\prime \prime \prime}=16 \pi / 21$ similarly as in Fig. 12 (a), (d). Finally, the smooth continuation of the linear SW with wave vector $Q$ towards smaller $C_{K}$ stops when approaching the third resonance from above (open circles in Fig. 12 (f)) at $C_{K} \approx 51.826293474688$. (As the third resonance is extremely weak, its correct numerical resolution requires working with quadruple precision and using a step size in $C_{K}$ smaller than $10^{-11}$.)

Let us finally note that in all cases, we find that for type $H \mathrm{SWs}$ in finite systems with periodic boundary conditions, stability will always be recovered in an interval close to the linear limit (c.f. Fig. 11 (d)), which shrinks to zero as the system size increases. The return to stability occurs through reentrant instabilities, completely analogous to the scenario for the DNLS approximation mentioned in Sec. 4.1. The shrinking of the stability regime with system size is illustrated in Fig. 13.

\subsection{The Morse potential}

For the soft Morse potential, the nonlinear SWs with wave vector $Q$ have frequencies $\omega_{b}<\omega_{l}(Q)$, and the continuation from the anticontinuous to the linear limit must be performed in two steps as illustrated in Fig. 5: first by increasing $C_{K}$ for fixed $\omega_{b}<1$, and then by increasing $\omega_{b}$ for fixed $C_{K}$. Even though the on-site potential (3) now is asymmetric, we can use the symmetry

\footnotetext{
${ }^{13}$ However, a smooth (but non-monotonous) continuation into a SW with wave vector $Q^{\prime \prime}=18 \pi / 21$, analogous to that in Fig. 12 (a), can be obtained in the following way [35]: at $C_{K} \approx 46.51932$, there is a bifurcation with a second solution, which can be followed smoothly by decreasing $C_{K}$ until (at $C_{K} \approx 46.51914$ ) it bifurcates with a third solution, which, again by increasing $C_{K}$, can be followed as it smoothly changes into the SW with wave vector $Q^{\prime \prime}$. This 'Z-type' behaviour, with the simultaneous existence of three different solutions for the same $\omega_{b}$ and $C_{K}$ in some regime, typically occurs in systems with effective ultra-long-range interactions yielding length-scale competition [36].
} 


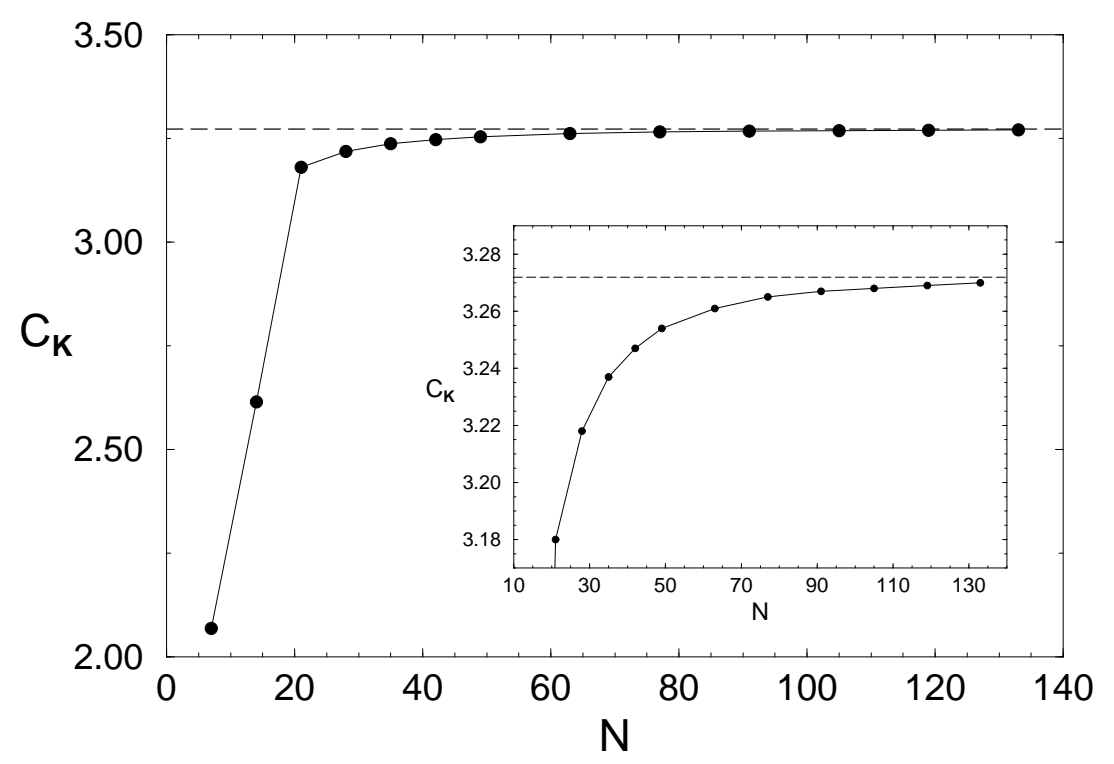

Fig. 13. Lower limit for the interval of stability close to the linear limit as a function of system size, for a type $H$ SW with $Q=4 \pi / 7$ and $\omega_{b}=3.0$ in a quartic KG lattice. The dashed line is the linear limit according to Eq. (8).

properties of the SW coding sequences discussed in Sec. 3.2 to restrict the space of solutions for the numerical Newton scheme. To find the time-reversible SWs of type $H$ with coding sequences antisymmetric around a lattice site we impose the conditions $u_{n}\left(T_{b} / 2\right)=u_{-n}(0), \dot{u}_{n}\left(T_{b} / 2\right)=\dot{u}_{n}(0)=0$ in the Newton algorithm, while for SWs antisymmetric around a bond center the first condition is replaced by $u_{n}\left(T_{b} / 2\right)=u_{-n+1}(0)$. For type $E$ SWs, the symmetry condition $u_{n}(t)=u_{-n+1}(t)$ can always be employed.

In the case of a soft potential, the $p$ th harmonic of a SW with wave vector $Q$ enters the linear phonon band if (c.f. (58))

$$
\frac{1}{p}<\omega_{b}<\min \left\{\sqrt{1+4 C_{K} \sin ^{2} \frac{Q}{2}}, \frac{1}{p} \sqrt{1+4 C_{K}}\right\} .
$$

Thus, in contrast to the hard case, we expect to find higher order resonances for all $Q$ if, for fixed $C_{K}$, the frequency $\omega_{b}$ is small enough, i.e., if the SW is sufficiently nonlinear. In particular, when the conditions (59) are fulfilled, the resonances occur immediately from the linear limit. As the potential is non-symmetric already the second-harmonic resonances yield generally bifurcations of the SWs, and thus we find qualitatively different behaviour for small-amplitude SWs depending on whether the condition (59) with $p=2$,

$$
Q<\frac{\pi}{3} \quad \text { and } \quad C_{K}>\frac{3}{4\left(1-4 \sin ^{2} \frac{Q}{2}\right)}
$$




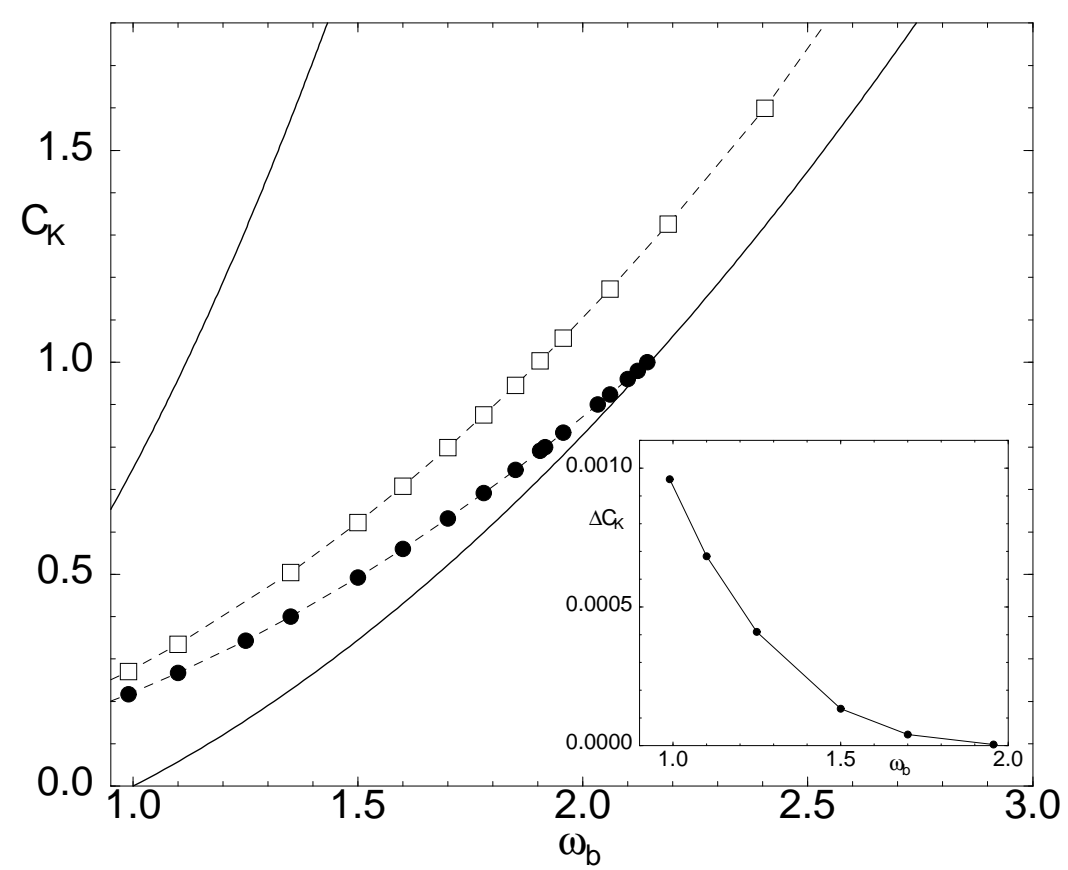

Fig. 14. Phase diagram for SWs with $Q=4 \pi / 5$ in the Morse KG model. The lower solid line is the linear dispersion curve (8), the filled circles indicate the line (which in fact are two closely separated lines, see text) of inversion of stability between the SWs of types $E$ and $H$ (characterized by eigenvalue collisions of $\mathbf{T}_{\mathbf{0}}$ at +1 ), and the open squares indicate the line of amplitude divergence. The upper solid line is the right boundary of the second-harmonic band. Inset shows the difference between the values of the coupling where the stability changes for the $H$ and $E$ waves for fixed frequency.

is fulfilled or not. In order to avoid complications resulting from higher-order resonances $(p>2)$, which occur only for highly nonlinear SWs, we will mainly concentrate the discussion here to the regime $\omega_{b}>1 / 2$ (more details concerning the behaviour for smaller $\omega_{b}$ are given in [35]). ${ }^{14}$

The typical behaviour when continuing SWs with $Q>\pi / 3$ is summarized in Figs. 14-15, for a rather simple case with $Q=4 \pi / 5$. Let us first concentrate on the part closest to the linear limit. Then, we find that increasing $C_{K}$ for a fixed $\omega_{b}$ close to 1 (which means that the wave amplitude also increases), the type $H$ SWs will suffer additional real instabilities, not present neither in the DNLS approximation nor in the quartic model. These instabilities occur through collisions at +1 at some critical value of the coupling. Very close to this value, also the type $E \mathrm{SW}$ with the same $Q$ and $\omega_{b}$ changes its

\footnotetext{
${ }^{14}$ In fact, the ansatz (28)-(29) for the SW coding sequences is valid only for $\omega_{b}>1 / 2$; a smooth continuation of SWs generated from (28)-(29) for $\omega_{b}>1 / 2$ towards smaller $\omega_{b}$ shows [35] that they appear with a different anticontinuous coding sequence when $\omega_{b}<1 / 2$, with the 0 's in the original code replaced by \pm 2 's. Solutions generated from the anticontinuous limit at $\omega_{b}<1 / 2$ using (28)-(29) are lost through bifurcations and not continuable to the linear limit [35].
} 

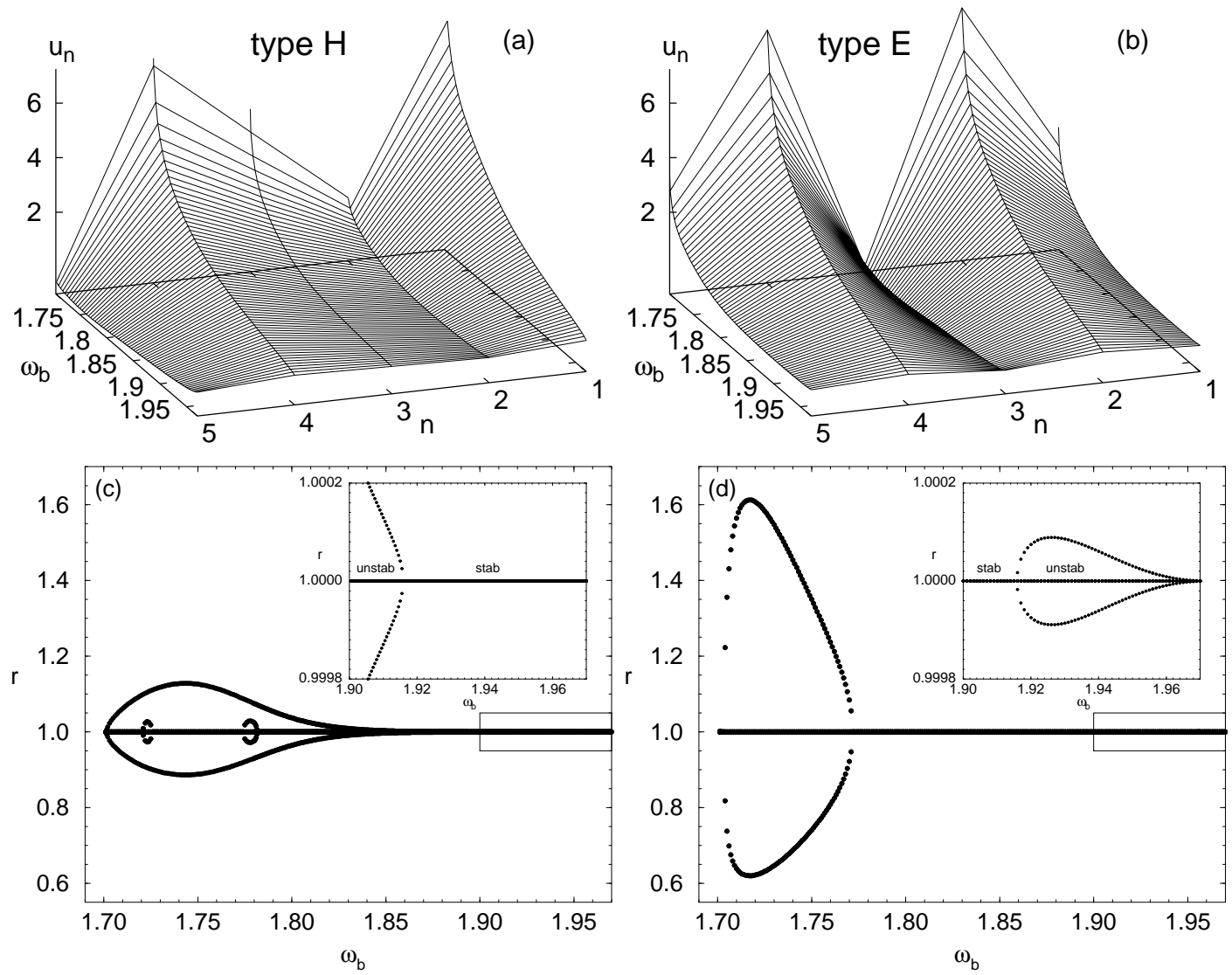

Fig. 15. Continuation from the linear limit at fixed coupling $C_{K}=0.8$ for decreasing frequency for (a),(c) the type $H$ and (b),(d) the type $E$ SW with $Q=4 \pi / 5$ in the Morse KG model (corresponding to a horizontal line in the phase diagram in Fig. $14)$. The wave amplitude diverges in both cases at $\omega_{b} \approx 1.701$. (c) and (d) show the magnitude of the eigenvalues $r \mathrm{e}^{i \theta}$ of $\mathbf{T}_{\mathbf{0}}$ corresponding to one unit cell $(N=5)$ of the $H$ and $E$ waves, respectively. Insets are magnifications of the region between the linear limit and the point of inversion of stability, which occurs at $\omega_{b} \approx 1.9155$. The other instabilities are oscillatory instabilities resulting from Krein collisions.

stability properties, in that the real eigenvalues which are normally present collide at +1 and go out on the unit circle, leaving only Krein instabilities and second-harmonic collisions at -1 as possible remaining instability mechanisms. This 'inversion of stability' is analogous to the scenario occurring between the single-site (code ...0001000...) and two-site (code ...0011000...) breathers in the Morse and other soft asymmetric potentials [37,38], and is generally associated with a highly increased mobility $[38,20,21]$. In the case shown in Figs. 14-15, the change of stability for the $E$-wave with a given frequency occurs for slightly smaller values of $C_{K}$ than that of the corresponding $H$-wave (see inset in Fig. 14), so that in the absence of other instability mechanisms (which is the case for small systems close to the linear limit, c.f. Fig. 15), there is a small regime in the $\left(\omega_{b}, C_{K}\right)$-plane of simultaneous stability for both waves ${ }^{10}$.

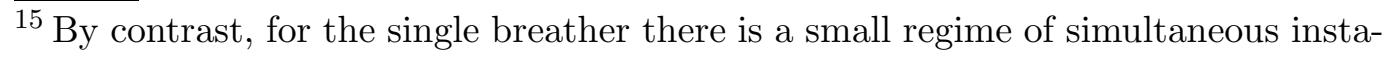


When increasing $\omega_{b}$ and $C_{K}$ the regime of simultaneous stability decreases, and moreover at a certain point $\left(\omega_{b} \approx 2.1\right.$ and $C_{K} \approx 1.0$ for the case in Fig. 14) the two lines defining the inversion of stability converge and intersect the linear dispersion curve (8). Thus, for values of $C_{K}$ above this point the stability scenario close to the linear limit is opposite to what we have found previously in the DNLS-approximation: the $H$-wave becomes immediately unstable through real eigenvalues, while the $E$-wave remains stable in an interval close to the linear limit for finite-size systems.

When further increasing the coupling, we find typically that the region of existence for the SWs is limited by a curve in the $\left(\omega_{b}, C_{K}\right)$-plane where the wave amplitude diverges as illustrated in Fig. 15 (a), (b) (as the Morse potential (3) becomes flat for large $u$ ). However, for smaller $\omega_{b}$ the scenario becomes more complicated with additional bifurcations, and we will not attempt to give an exhaustive analysis of this regime here (see [35] for more details). Let us just mention that for the case illustrated in Fig. 14 , when $\omega_{b} \lesssim 0.72\left(\omega_{b} \lesssim 1.1\right)$ for the $H$-wave ( $E$-wave), the continuation from the anticontinuous limit at fixed $\omega_{b}$ is interrupted 10 by a bifurcation before reaching the point of amplitude divergence. Let us also mention that for SWs with more complicated unit cells than that of Figs. 14-15 (which is somewhat special since the coding sequence for one unit cell of the $H$-wave [1-101-1] has only one 0 per unit cell, while that of the $E$-wave [-11-11-1] has no 0 and only one pair of consecutive -1), there are sometimes more than one 'stability-inverting' bifurcation, and the phase diagram of 'stable' (neglecting other instability mechanisms) and unstable regimes in the $\left(\omega_{b}, C_{K}\right)$-plane may contain more than two parts [35] (as was also found for the single breather [38]).

Let us now turn to the case with $Q<\pi / 3$, in which case we find secondharmonic resonances also in the linear regime for large enough coupling. The scenario in the small-amplitude regime is then similar to the scenario for the third-harmonic resonances in the quartic model illustrated in Fig. 12. An example for a type $H \mathrm{SW}$ with $Q=2 \pi / 11$ is shown in Fig. 16. Continuing from the anticontinuous limit for increasing coupling then yields (Fig. 16 (b)), via an eigenvalue collision at +1 for a value of $C_{K}$ on the line of open diamonds in (a), a transition to an intermediate state, which at a second bifurcation point becomes a SW with frequency $2 \omega_{b}$ and wave vector $Q^{\prime}$, where $Q^{\prime}$ is the wave vector closest to $\pi$ giving a SW whose symmetry is compatible with that of the original wave (here, the new wave is the type $E \mathrm{SW}$ with $\left.Q^{\prime}=10 \pi / 11\right) \square$.

$\overline{\text { bility [38]. }}$

${ }^{16}$ However, it can be restored [35] by following a curvilinear, 'Z-shaped' path similarly as described for the quartic model in footnote 13 . This behaviour is likely to be related to a strong effective long-range interaction in the extended DNLS equation (12); cf. [36].

${ }^{17}$ Similarly to the quartic model (footnote 12 ), a necessary resonance condition is $Q^{\prime}=m Q \bmod 2 \pi$, but with no restriction on the integer $m$. 

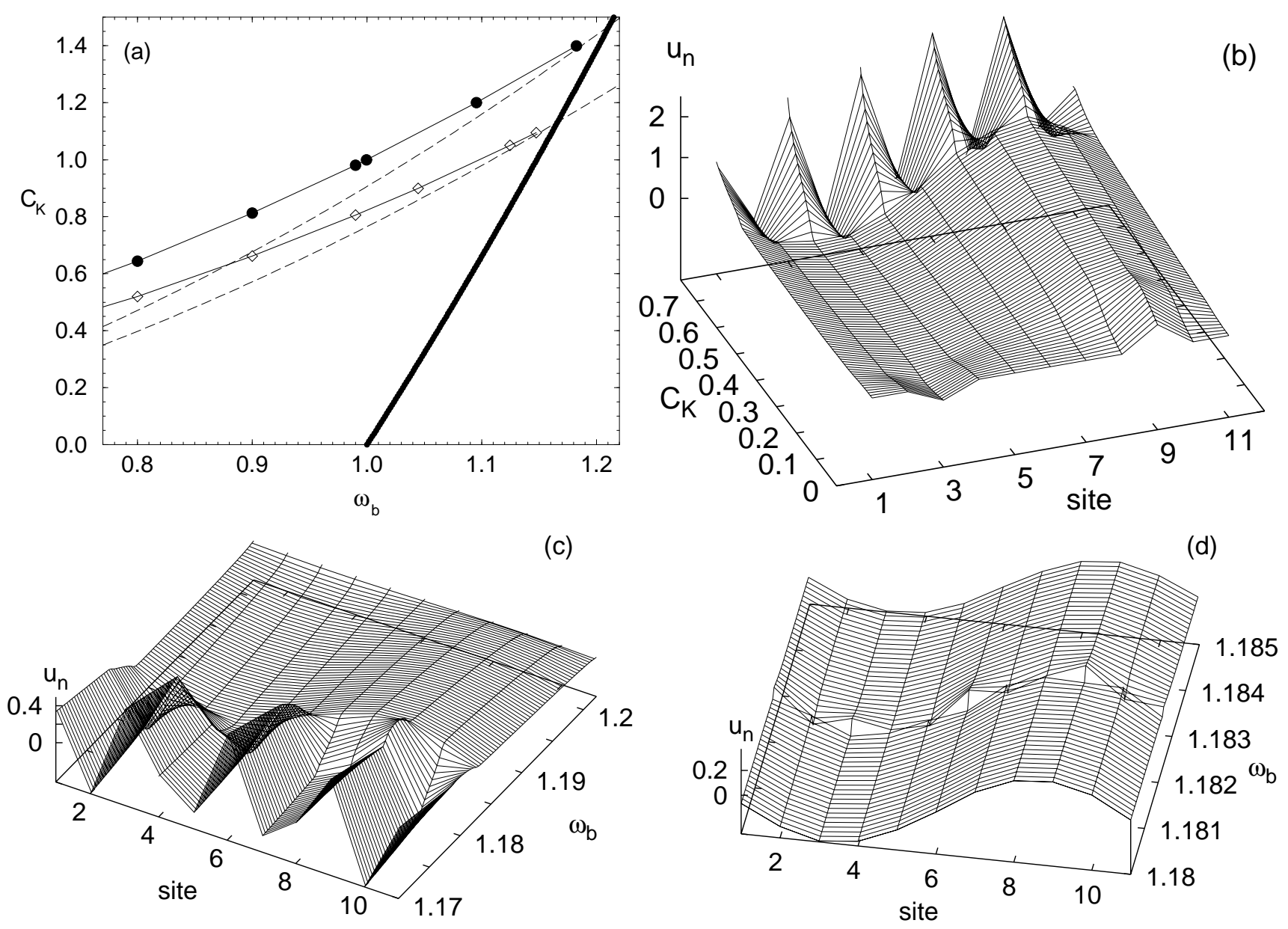

Fig. 16. Second-harmonic resonances for a type $H$ SW with $Q=2 \pi / 11$ in the Morse KG model. (a) shows the bifurcation lines for the first two resonances; dashed lines are the linear second-harmonic dispersion curves for $Q=10 \pi / 11$ (lower) and $Q=8 \pi / 11$ (upper); fat solid line is the fundamental linear dispersion curve for $Q=2 \pi / 11$. (b) illustrates the smooth continuation versus coupling from the anticontinuous limit at fixed frequency $\omega_{b}=0.9$ through the first resonance (open diamonds in (a)) into a type $E \mathrm{SW}$ with frequency $2 \omega_{b}$ and wave vector $Q^{\prime}=10 \pi / 11$. (c) shows smooth continuation versus frequency from the linear limit at constant coupling $C_{K}=1.4$ (i.e., between the first and second resonances) through the second resonance (filled circles in (a)) into a type $E$ SW with frequency $2 \omega_{b}$ and wave vector $Q^{\prime \prime}=8 \pi / 11$. (d) shows intentionally careless continuation which jumps over the resonance in (c); if a careful continuation versus larger $\omega_{b}$ is attempted, it stops at $\omega_{b} \approx 1.18235$ (filled circle in (a)) similarly as in Fig. $12(\mathrm{~d})$ and (e).

This new SW can then be continued for larger coupling until its amplitude diverges at some finite value of $C_{K}$ (similarly as in Fig. 15).

The same scenario is observed by continuation from the linear limit for fixed coupling towards lower frequency, as long as $C_{K}$ is below the value where the first resonance curve (which in the linear limit coincides with the linear second-harmonic dispersion curve for $Q=Q^{\prime}$, see Fig. 16 (a)) intersects the 
linear dispersion curve. For $C_{K}$ larger than this value the SWs are also continuable from the linear limit towards lower frequency, but attempts to continue these waves to the anticontinuous limit by subsequently decreasing $C_{K}$ fail since, similarly as for the quartic model (Fig. 12 (d), (e)), the continuation is interrupted when reaching the first resonance curve. Performing instead the continuation for fixed coupling, the scenario is completely analogous to that of smaller $C_{K}$ : as illustrated in Fig. 16 (c) for $C_{K}=1.4$, the wave bifurcates at a point on the line of filled circles in $(a)\left(\omega_{b} \approx 1.1816\right)$ into an intermediate state, which in a second bifurcation (at $\omega_{b} \approx 1.1789$ ) turns into the second-harmonic type $E$ SW with wave vector $Q^{\prime \prime}=8 \pi / 11$, which then can be continued until its amplitude becomes infinite. Similarly, if the continuation from the linear limit is performed for $C_{K}$ above the intersection point with this second resonance curve, the continuation towards smaller coupling will be interrupted by the second resonance curve, while the continuation towards larger coupling or smaller frequency meets a third resonance curve where the wave changes smoothly into a SW with wave vector $Q^{\prime \prime \prime}=6 \pi / 11$, etc. However, as illustrated by Fig. 16 (d), these resonances may be jumped and an apparently smooth continuation of the SW with the original wave vector $Q$ observed if the numerical continuation is performed taking large steps.

Finally, we remark that also in the case of second-harmonic resonances close to the linear limit, the inversion of stability between the $E$ and $H$ waves can be observed. For example, in the case considered in Fig. 16 (c), the first eigenvalue collision at +1 in the continuation from the linear limit occurs at $\omega_{b} \approx 1.1822$ (i.e., very close to the collision corresponding to the secondharmonic resonance), and corresponds to inversion of stability. Let us also remark that, as mentioned above, the bifurcation scenario for smaller $\omega_{b}$ becomes more complicated, and for example the monotonous continuation for fixed frequency versus coupling from the anticontinuous limit of the SW in Fig. 16 is interrupted before reaching the first second-harmonic resonance for $\omega_{b} \lesssim 0.7$.

\section{Dynamics}

Let us now discuss the dynamics resulting from the oscillatory instabilities of the SWs over different time ranges. We will here emphasize generic rather than model-dependent properties, and therefore we consider KG systems in the regime of small amplitude and small coupling, where the DNLS approximation is expected to be good and the model-dependent phenomena discussed in the previous section (i.e., higher-harmonic resonances and inversion of stability) do not play any role. As was illustrated already by Fig. 4 in [25], and is further exemplified by the figures in this Section, the dynamics in the regime of oscillatory instability can then typically be divided into roughly 


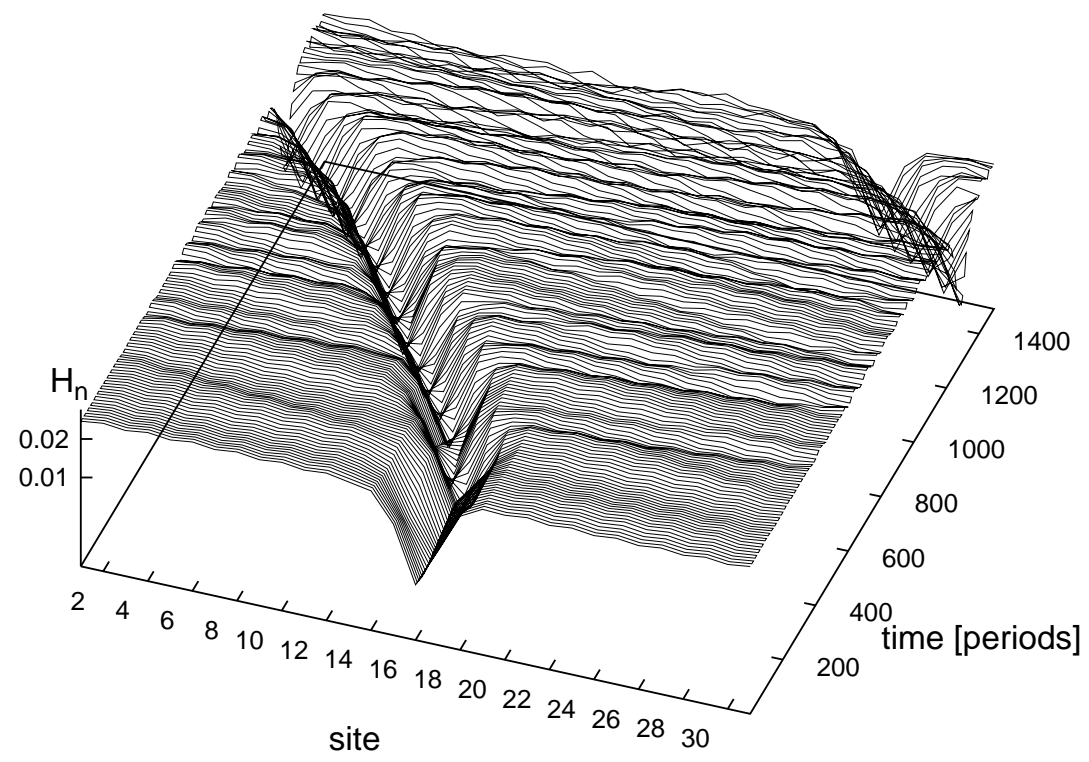

Fig. 17. Spontaneous motion (resulting only from numerical truncation errors) of a single discommensuration in a Morse potential KG chain (5) with $C_{K}=0.03$. The initial frequency is $\omega_{b}=1.036$.

three regimes. Initially, the small perturbation grows exponentially leading to oscillatory dynamics with small but increasing amplitude. As the oscillation amplitude reaches some threshold, an intermediate regime appears which typically is characterized by an inhomogeneous translational motion, where the wave remains locally coherent but with its different parts moving with respect to each other. Gradually, the wave loses coherence and a final, apparently chaotic state appears, the statistical properties of which depending crucially on the SW wave vector as discussed below.

To understand the appearance of the intermediate regime of translational motion, it is useful to first consider the case $Q \rightarrow \pi$ with one single discommensuration in the $Q=\pi$ phonon (for a soft potential). As was found in $[18,22]$ for the DNLS model, the oscillatory instability leads in this case to the motion of the discommensuration (for the DNLS model interpreted as a discrete 'grey' soliton) with a rather well-defined velocity for long times, although it continuously emits radiation and decays. The same type of behaviour occurs also in KG models for small $C_{K}$ as illustrated in Fig. 17, ए and is basically a consequence of the fact that the unstable eigenvector is spatially antisymmetric with respect to the background $Q=\pi$ wave around the discommensuration site. Thus, if we regard a SW with $Q$ close to $\pi$ as

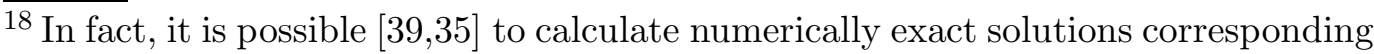
to moving discommensurations with given velocities, although they typically acquire an oscillatory extended tail and could therefore be described as 'dark nanopterons' (for the DNLS model, moving dark solitary waves close to the continuum limit have been numerically calculated in [40]).
} 

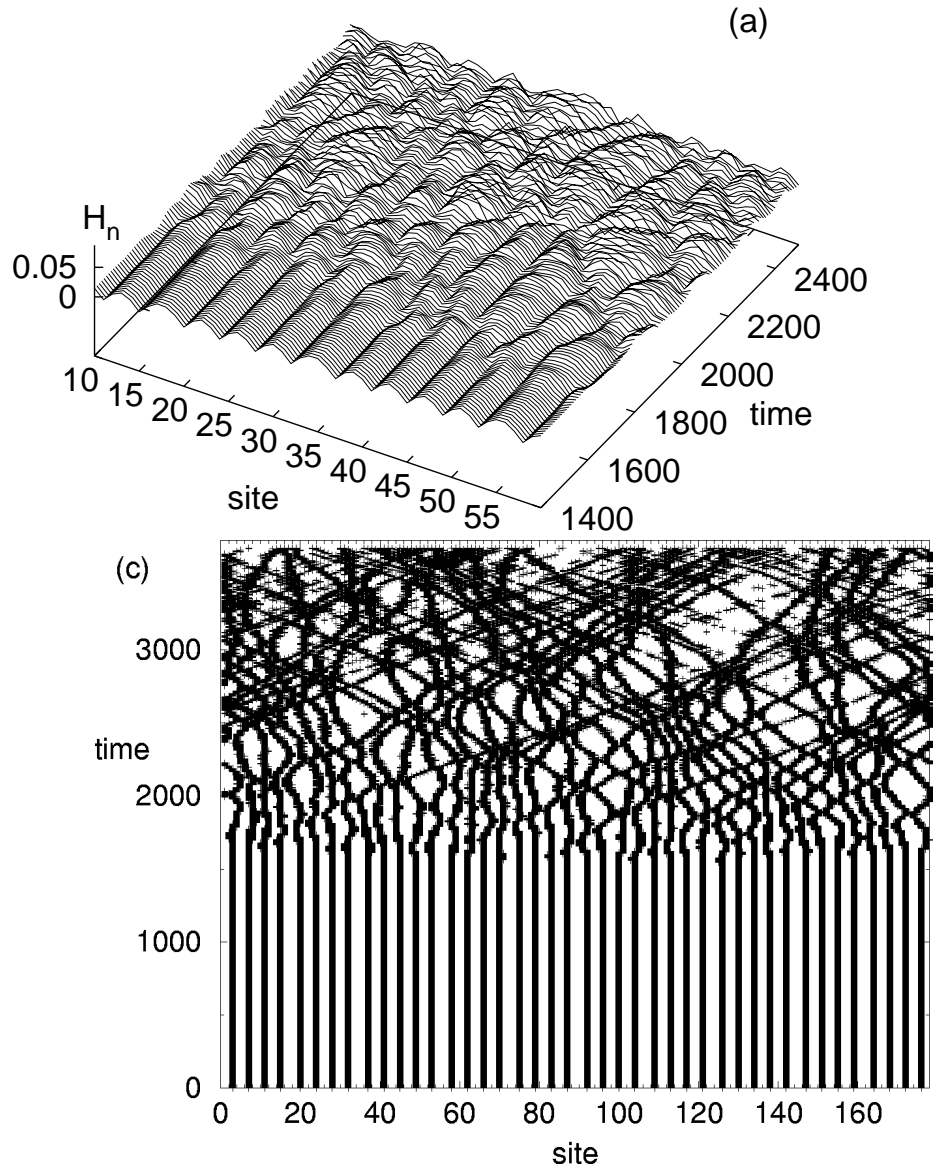
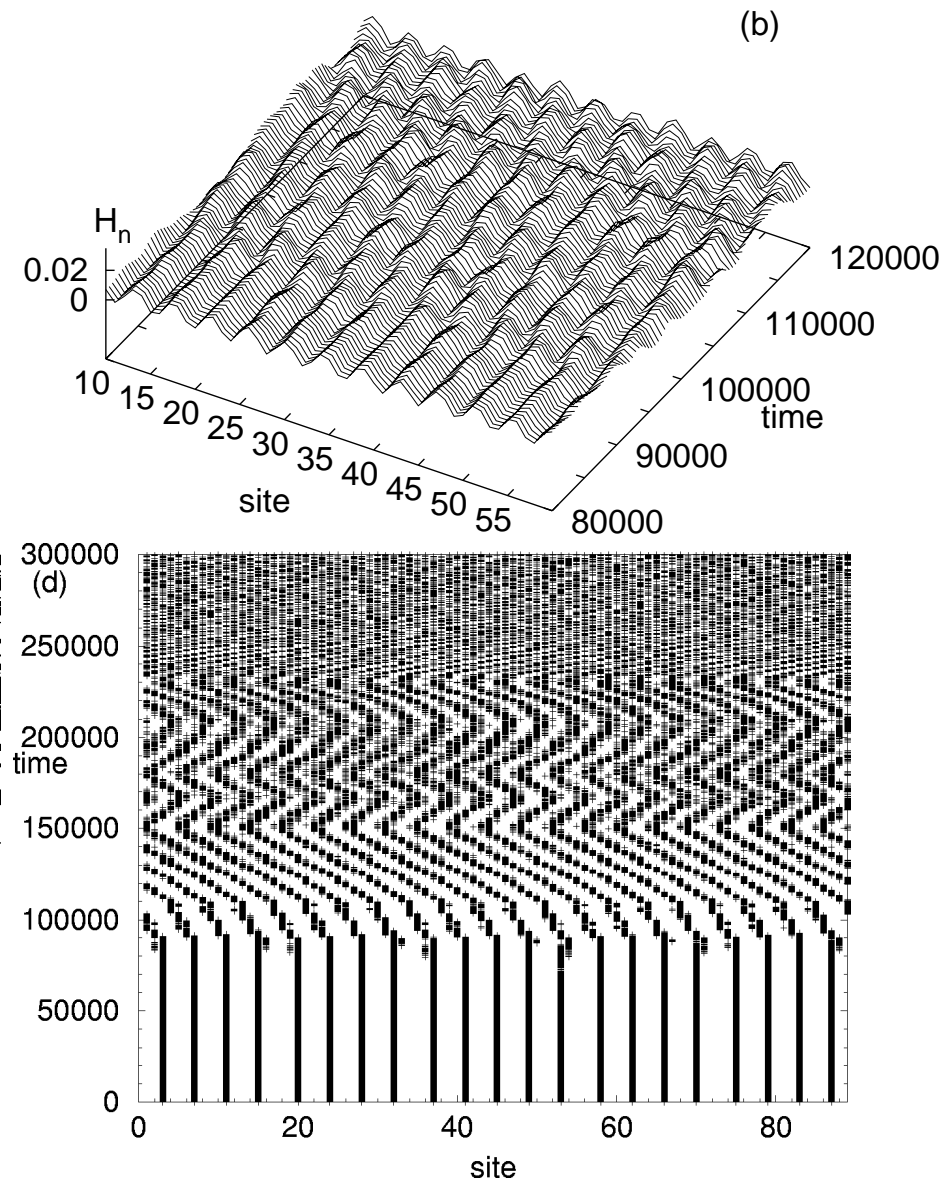

Fig. 18. Time evolution over short and intermediate time scales of (parts of) slightly perturbed SWs with $Q / 2 \pi=34 / 89 \approx \sigma_{G}$ in a Morse potential KG chain (5) with (a),(c) $C_{K}=0.03$ and $\omega_{b}=1.034$ (nonanalytic case, type $H$ ), and (b), (d) $C_{K}=0.05$ and $\omega_{b}=1.075$ (analytic case). (c) and (d) illustrate the time evolution and interaction of 'discommensurations', defined as sites where the energy is (c) $H_{n}<0.01$ respectively (d) $H_{n}<0.00232$ (corresponding to sites with code 0 for the initial SWs). Note the very slow evolution of the instability in the analytic case, which can be considered as 'quasistable' as discussed in Sec. 4.2: the strongest instability appears only to order $p=4$ according to Eq. (53).

an array of interacting discommensurations (where each discommensuration corresponds to a site with code 0 ), the intermediate regime of mainly translational motion can be anticipated. As illustrated in Fig. 18, this description is at least qualitatively relevant also for analytic waves (where generally discommensurations can no more be regarded as localized objects as their range of interaction diverges [19]) with wave vectors not necessarily close to $\pi$ (so that the discommensuration sites may be close). Initially, the collective movement of the discommensurations is well-ordered and they remain clearly separated from each other, but as time evolves their motion becomes more and more erratic and they start to collide and possibly merge with each other. Thus, the SW eventually loses its coherence. 

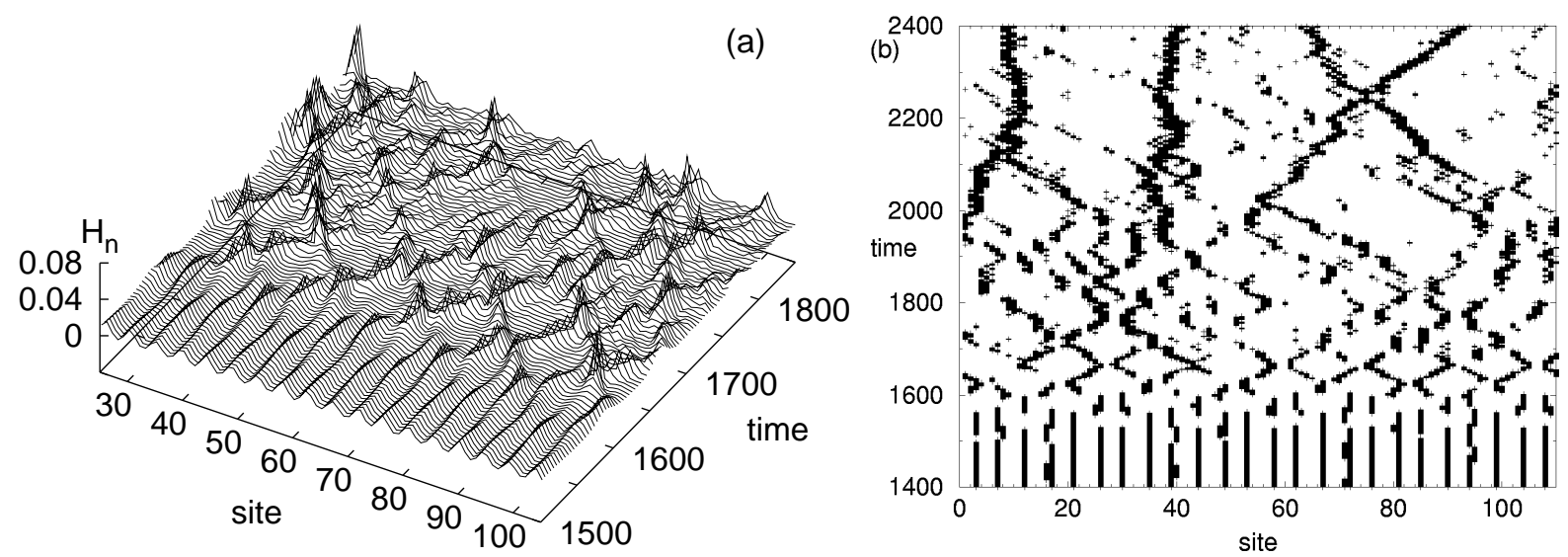

Fig. 19. Time evolution over short and intermediate time scales of (parts of) a slightly perturbed SW with $Q=12 \pi / 55(<\pi / 2)$ and $\omega_{b}=1.0015$ (corresponding to an analytic wave in the incommensurate limit) in a Morse potential KG chain (5) with $C_{K}=0.05$. (b) illustrates the time evolution and interaction of high-energy sites ('breathers'), defined as sites where the energy is $H_{n}>0.012$ (corresponding to sites with codes \pm 1 for the initial SW).

When (for a soft potential) $Q<\pi / 2$, the coding sequences contain consecutive zeros, and therefore the discommensurations in the initial SWs are not isolated. Thus, for small $Q$ it is more natural to regard the SWs as composed of individual breathers, where each breather corresponds to a site with code \pm 1 , and the distance between the breathers increases as $Q$ decreases. Then, as illustrated in Fig. 19, the intermediate regime can be interpreted as consisting of moving breathers which collide inelastically with each other.

Let us now discuss the nature of the asymptotic long-time states for different $Q$. For the DNLS equation (19), which is non-generic among nonlinear lattice equations in the sense that it has in addition to the Hamiltonian

$$
\mathcal{H}\left(\left\{\psi_{n}\right\},\left\{-\mathrm{i} \psi_{n}^{*}\right\}\right)=\sum_{n=1}^{N}\left(-\delta\left|\psi_{n}\right|^{2}+\frac{\sigma}{2}\left|\psi_{n}\right|^{4}+C\left|\psi_{n+1}-\psi_{n}\right|^{2}\right)
$$

also a second conserved quantity, the excitation norm

$$
\mathcal{N}=\sum_{n=1}^{N}\left|\psi_{n}\right|^{2}
$$

it has recently been shown [23] that the nature of the asymptotic dynamics for typical initial conditions depends critically on the values of these two conserved quantities. Redefining the Hamiltonian $(62)$ as $\mathcal{H}^{\prime}=\mathcal{H}+(\delta-2 C) \mathcal{N}$ (equivalent to applying the gauge transformation $\psi_{n} \rightarrow \psi_{n} \mathrm{e}^{-\mathrm{i}(\delta-2 C) t}$ to the conjugated 
variables $\left.\left\{\psi_{n}\right\},\left\{-\mathrm{i} \psi_{n}^{*}\right\}\right)$, a phase transition was found to occur along the line

$$
\frac{\mathcal{H}^{\prime}}{N}=\sigma\left(\frac{\mathcal{N}}{N}\right)^{2}
$$

in the thermodynamic limit $N \rightarrow \infty$. On one side of this line (below for $\sigma>0$ and above for $\sigma<0$ ), the system thermalizes in a standard Gibbsian sense with well-defined temperature and chemical potential, and the equilibrium probability distribution function (PDF) for $\left|\psi_{n}\right|^{2}$ decays rapidly for large $\left|\psi_{n}\right|^{2}$ so that large-amplitude excitations are highly improbable. However, on the other side of the line (64) the system exhibits a negative-temperature type behaviour where the stationary PDF for $\left|\psi_{n}\right|^{2}$ shows a positive curvature, corresponding to the creation of persistent localized high-amplitude standing breathers. The phase transition line corresponds to the limit of infinite temperature in the Gibbsian regime, and was obtained by assuming independent thermalization at each site, i.e., by neglecting the coupling term in the Hamiltonian. Now, we note that for a type $H$ SW with $Q=\pi / 2$ the coupling term is exactly zero, since according to the code sequence (29) such a wave must have the structure $\psi_{2 m+1}=(-1)^{m} \sqrt{\frac{\delta}{\sigma}}, \psi_{2 m}=0$ for all $\delta$. Thus, the $Q=\pi / 2 S W$ lies exactly on the phase transition line (64). Moreover, as illustrated in Fig. 20, this implies that type $H$ SWs with a given $Q$ always belong to the same well-defined phase regardless on the value of $\delta^{\prime}$, and that $S W s$ with $Q<\pi / 2$ and those with $Q>\pi / 2$ always belong to different phases.T By regarding a SW with wave vector $Q=\pi r^{\prime} / s^{\prime}$ as a multibreather with $r^{\prime}$ breathers (or, equivalently, $s^{\prime}-r^{\prime}$ discommensurations) in each (half-)period of $s^{\prime}$ sites, we obtain close to the anticontinuous limit (i.e., asymptotically for large $\mathcal{N} / N$ ): [ن]

$$
\frac{\mathcal{H}^{\prime}}{N} \approx \sigma \frac{s^{\prime}}{2 r^{\prime}}\left(\frac{\mathcal{N}}{N}\right)^{2}+2 C \frac{2 r^{\prime}-s^{\prime}}{r^{\prime}} \Theta\left(2 r^{\prime}-s^{\prime}\right)\left(\frac{\mathcal{N}}{N}\right)
$$

where $\Theta(x)$ is the usual step function.

Thus, within the DNLS approximation and assuming a soft potential $(\sigma=$ -1 ), we should expect for large systems to always observe an equipartitioned asymptotic state for all initially slightly perturbed unstable type $H$ SWs with $Q>\pi / 2$, while persistent breathers should be created from SWs with $Q<$ $\pi / 2$ (with the opposite conclusion for hard potentials). We confirmed this by numerical simulations, although for $Q$ close to but smaller than $\pi / 2$ the system size $N$ needs to be rather large to allow breather formation. Moreover, we confirmed numerically that also for the original KG lattices there appears to be a phase transition at $Q=\pi / 2$ between a thermalized phase and a 'breather

\footnotetext{
${ }^{19}$ Note that this is not in general true for propagating waves, which for a given wave vector $Q$ can cross the transition line (64) as the amplitude is varied [23].

20 This expression is exact for $Q=\pi / 2$ and $Q=\pi$.
} 


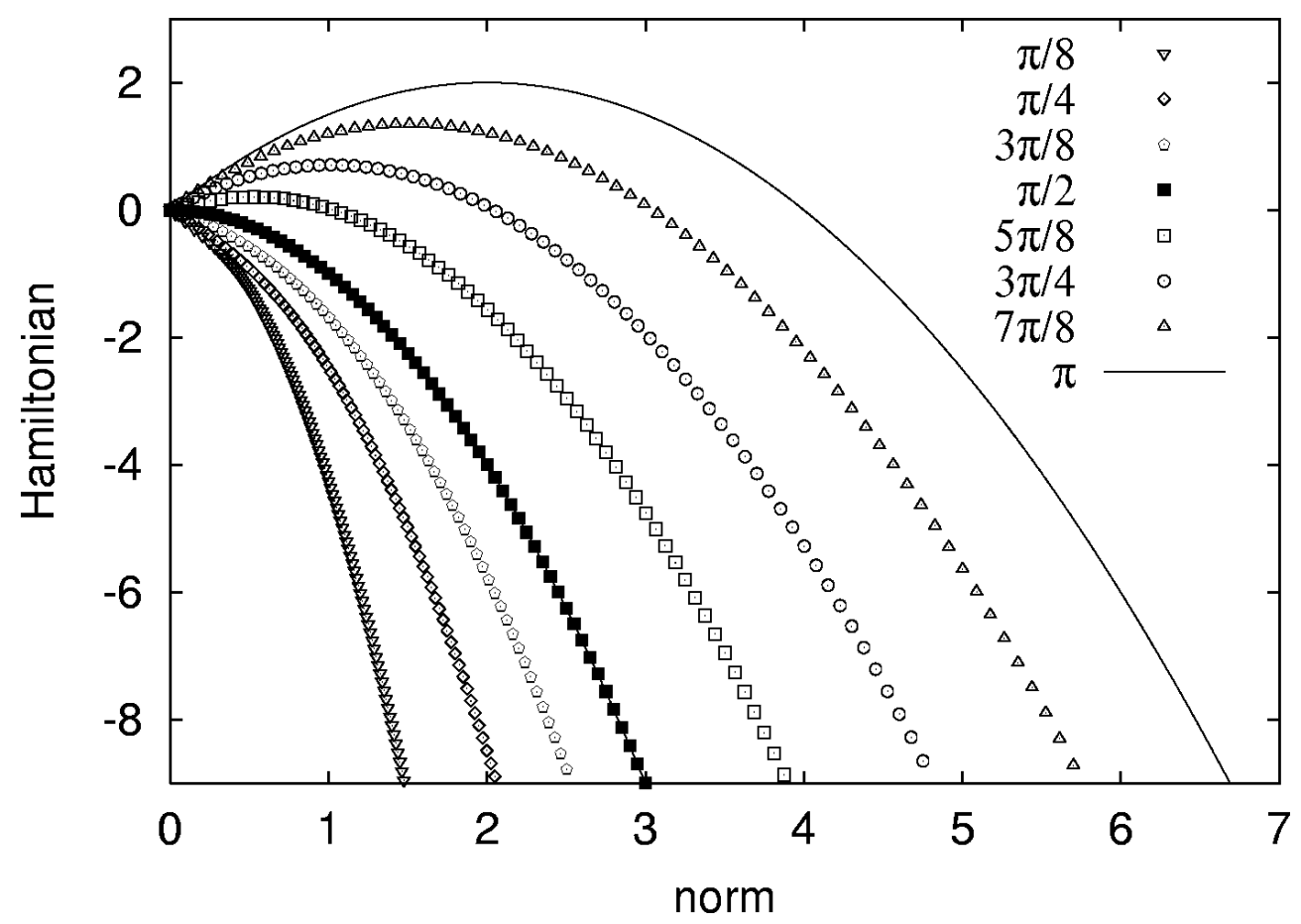

Fig. 20. Average Hamiltonian $\mathcal{H}^{\prime} / N$ versus average norm $\mathcal{N} / N$ for type $H$ DNLS SWs $(\sigma=-1, C=1)$ having wave vectors (increasing from left to right) $Q=\pi / 8$, $\pi / 4,3 \pi / 8, \pi / 2,5 \pi / 8,3 \pi / 4,7 \pi / 8$, and $\pi$. The points corresponding to $Q=\pi / 2$ (filled squares) coincide exactly with the phase transition line (64).
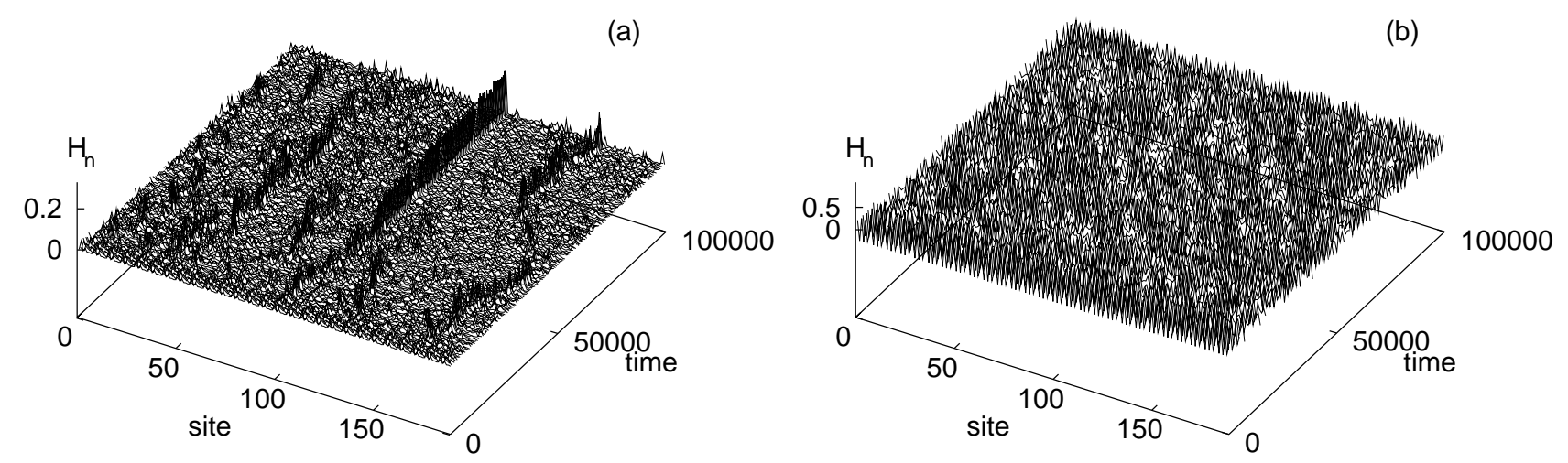

Fig. 21. Long-time dynamics of slightly perturbed unstable type $H$ SWs in a Morse KG lattice with $C_{K}=0.035$ and $N=189$. (a) shows breather formation for $Q=2 \pi / 7<\pi / 2$ and $\omega_{b}=0.995$, while (b) shows thermalization for $Q=6 \pi / 7>\pi / 2$ and $\omega_{b}=1.035$.

phase' (Fig. 21). However, it should be stressed that the results derived in [23] depend crucially on the existence of a second conserved quantity, and it is therefore questionable whether such a transition strictly speaking occurs also in other models, or if the 'breather phase' observed here for KG models only describes the dynamics over a long but finite time range, while asymptotically thermalization will occur also there. In any case, over the time scales that we have been able to follow numerically we have seen no signs of breather 
decay, and thus it is clear that the phase transition obtained in the DNLS approximation is of large relevance also for the long-time dynamics in the KG models.

\section{Conclusions}

In conclusion, we have shown that for a general class of anharmonic lattices, different types of SWs with spatial period commensurate or incommensurate with the lattice exist, but are generically unstable through oscillatory instabilities for small amplitudes. Thus, in discrete systems, the nonlinearity-induced coupling between counter-propagating waves leads to wave break-down for arbitrarily weak anharmonicity even if the individual propagating waves are modulationally stable. The physical mechanisms of the oscillatory instabilities can be related to a two-component structure of the SWs, which however is of different nature close to the anticontinuous and to the linear limits, respectively. In the first case, the natural interpretation is in terms of the multibreather description (28)-(29) of the SW, with (quasi-)periodically repeated sites of large (code \pm 1 ) and small (code 0 ) amplitude, which defines a twosublattice structure. Then, for small inter-site coupling the eigenmodes of the linearized equations describing the small-amplitude oscillations around the exact SW are mainly localized on one of these two sublattices, and the instabilities appear when, as the coupling is increased, eigenfrequencies belonging to the different sublattices start to coincide. On the other hand, close to the linear limit, the oscillatory instabilities arise from overlap between positiveand negative-frequency plane-wave solutions to the linearized equations (32) in the DNLS-approximation, which are coupled by the SW amplitude $\psi_{n}^{2}$. ${ }^{21}$

It is instructive to point out some aspects where the stability analysis of SWs considerably differs from that of propagating waves. Firstly, for propagating waves there is no two-component structure at the anticontinuous limit, as their amplitude is homogeneous with all sites having codes $\left|\tilde{\sigma}_{n}\right|=1$ [7]. Secondly, propagating waves are non-time-reversible with a complex amplitude $\psi_{n}=\mathrm{e}^{\mathrm{i} Q n}$ in the DNLS approximation, which modifies the eigenvalue problems (32)-(33) [31]. Thirdly, the spatial Fourier expansion (42) of a propagating wave in the DNLS approximation trivially contains only one term $(p=1)$, so that instabilities resulting from higher-order resonances (which are the only instabilities appearing for analytic incommensurate DNLS SWs close to the linear limit) are excluded.

\footnotetext{
${ }^{21}$ The existence of a regime of two-channel linear phonon scattering in the DNLS equation was also discussed in Ref. [41]; however, in contrast to what was claimed in these papers this regime does not exist for a single localized breather (the overlap described by Eq. (35) vanishes as $Q \rightarrow 0$ ).
} 
We should also stress that in general, there is no direct relation between the frequency of the SW and the frequencies of the oscillatory instabilities, as the instabilities are related to resonances between two different classes of solutions to the linearized equations (with opposite Krein signature), and not to the SW frequency itself. Also, as the instabilities for the infinite chain result from overlap between continuous bands and not isolated eigenvalues, the wavelengths of the unstable modes generally belong to a broad spectrum. However, for commensurate waves close to the linear limit, we proved in the Appendix that the first-order (strongest) instabilities correspond to frequencies and wavelengths close to, but different from, those of the SW itself $(\omega \approx 0,|q| \approx Q$, cf. Eq. (A.26)). Moreover, we showed by perturbation theory that the strength of the oscillatory instability (i.e., its growth rate) close to the linear limit is proportional to the corresponding spatial Fourier component $\left|f_{p}\right|$ of its squared DNLS amplitude $\psi_{n}^{2}$, and thus initially increases with the amplitude. The instability persists until, in the highly nonlinear regime, the frequencies of the two subclasses of linearized modes become out of resonance (through an inverse Krein bifurcation), so that close to the uncoupled limit the instability is suppressed (cf. the similar scenario for the discrete dark soliton corresponding to $Q \rightarrow \pi$ in [18]).

Obviously, this paper has been mainly concerned with fundamental properties of the SWs and their instabilities in generic but simple models, and more work needs to be done in order to elucidate their role in real physical systems, e.g., whether they have some influence on thermal transport coefficients. However, one result obtained here (Sec. 6) that should be emphasized in this context is, that the partition of the phase space for the asymptotic dynamics of the DNLS model (due to the norm conservation) in two regimes found in Ref. [23], corresponding respectively to a Gibbsian thermal equilibrium and formation of persisting breathers, is given a clear physical interpretation in terms of the SWs: the transition line (64) is defined by a (type $H$ ) SW with wave vector $Q=\pi / 2$, and SWs with given $Q$ always belong to the same regime regardless of their amplitude. More work should be done in order to elucidate the relevance of this transition for the KG system, e.g., to see if the partition of the phase space is fully broken in the absence of norm conservation, and to determine typical lifetimes of 'special' configurations like breathers and SWs. In this context, we also want to draw the attention to the very recent Ref. [42], where the processes of breather formation, coalescence and evolution to energy equipartition were analyzed for the FPU chain. There, it was found that exciting initially a single linear mode, the initial state first relaxes to an anharmonic spatially periodic solution, which we interpret as the FPU counterpart of our SWs. Later, this solution breaks up and the periodicity is lost, which we believe is related to instabilities similar to those obtained in our work. Thus, the SWs and their instabilities apparently provide an important link in the understanding of the pathway to energy localization and equipartition in anharmonic lattices. 
Concerning possible experimental observation, the general nature of our results suggests that these instabilities should be observable in macroscopic as well as microscopic contexts. In particular, as was recently experimentally verified [43], nonlinear optical waveguide arrays provide a direct application of the DNLS equation, and thus these systems are also good candidates for detection of SW instabilities.

\section{Acknowledgements}

G. Iooss and K. Kirchgässner are acknowledged for sending us their preprints prior to publication. We also thank A.J. Lichtenberg for discussions, and the two referees for useful remarks. GK thanks CNRS and LLB for their hospitality. MJ acknowledges a Marie Curie Research Training Grant from the European Community.

\section{A Linear stability analysis of small-amplitude standing waves close to double resonances}

\section{A.1 Points of simultaneous resonances}

Consider the ellipses (52) describing, for each $p$, the points in the $(\omega, E)$ plane where $p$ th order resonances of the second kind (48) occur. On each such ellipse, there are special points where also the first resonance condition (47) is fulfilled, so that both the above resonances exist simultaneously. These points are obtained for special values of $q$ in the parametrized representation of the ellipse $\left(\omega_{p}(q), E_{p}(q)\right)$ defined by Eqs. (50) and (51) with parameter $q=p^{\prime} Q$ ( $p^{\prime}$ integer). Then, a gap with a width of the order of $\left|f_{p^{\prime}}\right|$ opens in the ellipse, which makes this perturbed ellipse discontinuous. Thus, even if the ellipse (52), describing the center of the $p$ th order gap openings of the second kind, intersects the real axis $E=0$ so that an instability should be expected (i.e., if condition (53) is fulfilled), this disconnected curve might not intersect the real axis if $E=0$ is close to a point of simultaneous resonance, so that there is not necessarily an instability. However, since $f_{p}$ decreases exponentially as a function of $p$, only the gaps generated for $\left|p^{\prime}\right| \leq|p|$ makes a significant discontinuity in the ellipse of $p$ th order gaps. Thus, as the number of significant discontinuities in each ellipse is finite, we expect that only in exceptional cases a predicted gap opening around $E=0$ might be prohibited, and consequently the condition (53) for $p$ th order instability should be generic.

The values of $Q$ for which simultaneous resonances of orders $p^{\prime}$ and $p$ occur 
on the axis $E=0$ are obtained by vanishing Eq. (51):

$$
2 C-\delta-2 f_{0}-C \cos \left(p^{\prime}+2 p\right) Q-C \cos p^{\prime} Q=0 .
$$

In the zero-amplitude limit $f_{0}=0, \delta=\delta_{0}(Q)$ (Eq. (24)), this condition reduces to

$$
\cos Q=\cos p Q \cos \left(p+p^{\prime}\right) Q
$$

which is only fulfilled for a discrete set of values of $Q$ when $|p|>1$. However, for the ellipse (54) with $p=1$, the condition (A.2) is fulfilled for all $Q$ when $p^{\prime}=-1$. Thus, in the zero-amplitude limit there is always a point of double first order resonances at $\omega=0$ where the corresponding ellipse is tangent to the axis $E=0$. Consequently, we should treat this case separately as done below.

We will here discuss both commensurate and incommensurate SWs. In the commensurate case with $Q=\pi r^{\prime} / s^{\prime},\left\{\psi_{n}^{2}\right\}$ is an $s^{\prime}$-periodic sequence, and $2 p Q$ modulo $2 \pi$ takes only $s^{\prime}$ different values. Then, the Fourier expansion (42) of $\psi_{n}^{2}$ will be a finite sum, where $|p|$ runs only from 0 to $s^{\prime} / 2$ when $s^{\prime}$ is even, and to $\left(s^{\prime}-1\right) / 2$ when $s^{\prime}$ is odd. Consequently, there is only a finite number of ellipses (52) in the commensurate case. Furthermore, it follows from Eq. (29) and the model symmetries that the sequence $\left\{\psi_{n}^{2}\right\}$ for a periodic cycle will be symmetric either around a lattice site, in which case we choose $\psi_{n}^{2}=\psi_{-n}^{2}$, or around a bond center, so that we choose $\psi_{n}^{2}=\psi_{-n+1}^{2}{ }^{22}$ In the first case, we have the phase $\phi=0$ in Eq. (42), so that $f_{p}=f_{-p}$ are real. In the second case, $\phi=-Q / 2$ in (42) so that $f_{p}=f_{-p}^{*}$ in general would be complex, but by redefining the Fourier transforms (40) and (42) by changing $n \rightarrow n-\frac{1}{2}$ we again have $\phi=0$ and $f_{p}=f_{-p}$ real.

\section{A.2 General properties of the band spectrum at $\omega=0$}

Let us consider the eigenequation (38) for the special parameter value $\omega=0.23$ Then, it splits into two independent eigenequations which can be solved either with $W_{n} \equiv 0$ or $U_{n} \equiv 0$ :

$$
\begin{gathered}
\mathcal{L}_{1} U_{n}=\left(2-\delta-3 \psi_{n}^{2}\right) U_{n}-U_{n+1}-U_{n-1}=E U_{n} \\
\mathcal{L}_{0} W_{n}=\left(2-\delta-\psi_{n}^{2}\right) W_{n}-W_{n+1}-W_{n-1}=E W_{n} .
\end{gathered}
$$

$\overline{22}$ Both symmetries may also exist simultaneously, c.f. the discussion in Sec. 3.2.

${ }^{23}$ For convenience, in the rest of this Appendix we assume $C=1$ and $\sigma=-1$ without loss of generality. 
Eq. (A.3) can be related to the nonlinear symplectic map (23), for which the linearized equation is

$$
\mathcal{L}_{1} \epsilon_{n}=\left(2-\delta-3 \psi_{n}^{2}\right) \epsilon_{n}-\epsilon_{n+1}-\epsilon_{n-1}=0
$$

where $\epsilon_{n}$ is real and time constant. As well as for Eq. (30), we can associate with this linear equation a real eigenvalue problem defined by $\mathcal{L}_{1} \epsilon_{n}=E \epsilon_{n}$, which is nothing but Eq. (A.3). It was shown very generally in [44] that a necessary and sufficient condition for a trajectory $\psi_{n}$ of a symplectic map to be uniformly hyperbolic is that $E=0$ does not belong to the spectrum of the operator defined by the second variation of the action, which in our case is $\mathcal{L}_{1}$.

When $\psi_{n}$ is space periodic, and thus represented by a periodic cycle in the map defined by Eq. (23), the spectrum of Eq. (A.3) consists of bands separated by gaps. Then, if (and only if) the cycle is hyperbolic $E=0$ belongs to a gap, if it is parabolic 0 is at the edge of a gap, while for an elliptic cycle 0 belongs to the inner of a band. When $\left\{\psi_{n}\right\}$ is represented by a cantorus in the map (23), the corresponding trajectory is uniformly hyperbolic and then 0 does not belong to the spectrum of Eq. (A.3). On the other hand, when it is represented by a KAM torus 0 belongs to the spectrum, and moreover there is an explicit eigenvector for this eigenvalue. In that case, $\psi_{n}$ is a quasiperiodic function of $n$ with an analytic hull function $\chi_{S}(x)$ defined by Eq. (27), and Eq. (A.3) exhibits the solution $U_{n}=\partial \chi_{S}(Q n+\phi) / \partial \phi$ (the sliding mode) for the eigenvalue $E=0$.

The second eigenequation (A.4) has always the explicit solution $W_{n}=\psi_{n}$ (the phase mode) for the eigenvalue $E=0$. When $\psi_{n}^{2}$ is space periodic with period $s^{\prime}$, the eigensolutions of Eq. (A.4) are Bloch states with a wave vector $q_{s^{\prime}}$ defined modulo $2 \pi / s^{\prime}$, and the corresponding eigenvalues form bands separated by gaps. Since the solution $W_{n}=\psi_{n}$ has the wave vector $q_{s^{\prime}}=0$ or $\pi$ modulo $2 \pi / s^{\prime}$, its corresponding eigenvalue $E=0$ is at the edge of a gap of the spectrum of Eq. (A.4). When $\left\{\psi_{n}\right\}$ is space quasiperiodic and represented by a KAM torus in the map (23), the spectrum of Eq. (A.4) is a Cantor set with infinitely many gaps. Again, $E=0$ is at an edge of a gap of this Cantor spectrum.

\section{A.3 First-order perturbative approach for $\omega$ and $E$ close to zero}

We now take advantage of these informations for studying the spectrum of Eq. (38) close to the point of double first-order resonances $(\omega, E) \approx(0,0)$ in the limit when the SW amplitude $\psi_{n}$ is small and thus close to a cosine function. Then, at the lowest order $p=1$, it is sufficient to assume that $f_{0} \neq 0$, 
$f_{1}=f_{-1} \neq 0$ and $f_{p}=0$ for $|p| \geq 2$. In analogy with Eq. (43) we set

$$
f_{0}=2 f_{1}+\alpha
$$

where $|\alpha| \ll f_{0}$ but in general not strictly zero. Then, for describing consistently at leading order the band structure in the neighbourhood of the origin in the $(\omega, E)$-plane, we should essentially consider the reduction of Eq. (44) to the subspace involving the four almost degenerate eigenvectors which are the two components with wave vector $q$ and the two components with wave vector $q+2 Q$ with $q \approx-Q$. Then, close to the origin $\omega=0, E=0$, the bands of eigenvalues $E_{\lambda}(q ; \omega)$ are well described by diagonalization of the $4 \times 4$-matrix

$$
\mathbf{M}(q ; \omega)=\left(\begin{array}{cccc}
a(q)-f_{0} & -\omega & -3 f_{1} & 0 \\
-\omega & a(q)+f_{0} & 0 & -f_{1} \\
-3 f_{1} & 0 & a(q+2 Q)-f_{0} & -\omega \\
0 & -f_{1} & -\omega & a(q+2 Q)+f_{0}
\end{array}\right)
$$

where

$$
a(q)=2-\delta-2 f_{0}-2 \cos q
$$

\section{A.3.1 Spectrum at $\omega=0$}

When $\omega=0$, we should recover from Eq. (A.7) the properties of the spectrum of Eq. (A.3) associated with the tangent map (A.5), as well as the phase mode of Eq. (A.4). Then, the $4 \times 4$ matrix (A.7) splits into two diagonal blocks of $2 \times 2$ matrices,

$$
\mathbf{L}_{S}(q)=\left(\begin{array}{cc}
a(q)-f_{0} & -3 f_{1} \\
-3 f_{1} & a(q+2 Q)-f_{0}
\end{array}\right)
$$

which yields the spectrum of (A.3) calculated in perturbation for $q$ close to $-Q$ as

$$
\begin{aligned}
E_{S \pm}(q)= & 2-\delta-3 f_{0}-\cos q-\cos (q+2 Q) \\
& \pm \sqrt{(\cos q-\cos (q+2 Q))^{2}+9 f_{1}^{2}}
\end{aligned}
$$


and

$$
\mathbf{L}_{P}(q)=\left(\begin{array}{cc}
a(q)+f_{0} & -f_{1} \\
-f_{1} & a(q+2 Q)+f_{0}
\end{array}\right)
$$

which yields the spectrum of (A.4) as

$$
\begin{aligned}
E_{P \pm}(q)= & 2-\delta-f_{0}-\cos q-\cos (q+2 Q) \\
& \pm \sqrt{(\cos q-\cos (q+2 Q))^{2}+f_{1}^{2}}
\end{aligned}
$$

The phase mode at $E=0$ corresponds to the eigenvector $(1,1)$ of the matrix (A.11) at $q=-Q$, which implies

$$
E_{P-}(-Q)=2-\delta-f_{0}-f_{1}-2 \cos Q=0,
$$

or

$$
\delta=\delta_{0}(Q)-f_{0}-f_{1}
$$

Then, we have

$$
\begin{gathered}
E_{P+}(-Q)=2 f_{1}>0, \\
E_{S+}(-Q)=-2\left(f_{0}-2 f_{1}\right) \equiv-2 \alpha,
\end{gathered}
$$

and

$$
E_{S-}(-Q)=-2\left(f_{0}+f_{1}\right)<0
$$

For an analytic incommensurate SW, the sliding mode at $E=0$ corresponds to the eigenvector $(1,-1)$ of the matrix $($ A.9) at $q=-Q$. Then, vanishing Eq. (A.16) yields $\alpha=0$ so that $f_{0}=2 f_{1}$, and Eq. (A.13) then yields $2 \cos Q=$ $2-\delta-3 f_{0} / 2$.

Otherwise, as explained in Sec. A.2, for commensurate SWs represented by hyperbolic periodic cycles $E=0$ does not belong to the spectrum of the matrix (A.9) for any $q$ close to $-Q$, while for elliptic cycles $E=0$ is in the spectrum of (A.9) for some values of $q$ close to (but away from) $-Q$ (as $E=0$ is not at a band edge). In both cases, Eq. (A.16) yields that $\alpha \neq 0$. Defining $\xi$ as $q=\xi-Q, \xi$ small and real, it follows from Eq. (A.10) that $E=0$ is in the spectrum of (A.9) if and only if the equation 


$$
\begin{array}{r}
E_{S+}(\xi-Q) E_{S-}(\xi-Q)=4 \cos ^{2} \xi-4\left(2-\delta-3 f_{0}\right) \cos Q \cos \xi \\
+\left(2-\delta-3 f_{0}\right)^{2}-9 f_{1}^{2}+4 \cos ^{2} Q-4=0
\end{array}
$$

has a solution for $\xi$ real. With $\cos Q$ taken from Eq. (A.13), this equation becomes using (A.6)

$$
\begin{array}{r}
(1-\cos \xi)\left(2+2 \cos \xi-\left(2-\delta-3 f_{0}\right)\left(2-\delta-3 f_{0} / 2+\alpha / 2\right)\right) \\
=\alpha\left(3 f_{0}-\alpha\right)
\end{array}
$$

At leading order for $\xi$ small and $f_{0}$ small, Eq. (A.19) yields

$$
\frac{1}{2} \xi^{2}\left(4-(2-\delta)^{2}\right)=\alpha\left(3 f_{0}-\alpha\right)
$$

Since $0<\delta<4$ for all $Q \neq 0$ if $f_{0}$ is small enough, there is a real solution for small $\xi$ only when $\alpha \geq 0$. ${ }^{24}$ Consequently, as a perturbative result valid at small amplitude, $E=0$ belongs to the spectrum of (A.9) for some real $|\xi|>0$ when $\alpha>0$, and does not when $\alpha<0$. Thus, the commensurate SW is represented by an elliptic periodic cycle when $f_{0}>2 f_{1}$ and a hyperbolic periodic cycle when $f_{0}<2 f_{1}$.

\section{A.3.2 Band intersection with $E=0$}

The determinant of the matrix (A.7) is

$$
\begin{array}{r}
D(q ; \omega)=\omega^{4}-\omega^{2}\left(a^{2}(q)+a^{2}(q+2 Q)-2 f_{0}^{2}+6 f_{1}^{2}\right) \\
+\left(\left(a(q)-f_{0}\right)\left(a(q+2 Q)-f_{0}\right)-9 f_{1}^{2}\right) \times \\
\left(\left(a(q)+f_{0}\right)\left(a(q+2 Q)+f_{0}\right)-f_{1}^{2}\right) .
\end{array}
$$

Vanishing $D(q ; \omega)$ yields the intersections with the axis $E=0$ of the perturbed bands at wave vector $q$ close to $-Q$. Thus, stability requires the zeros $\omega_{s}(q)$ of $D(q ; \omega)$ to be real for all wave vectors $q$ close to $-Q$. At $q=-Q$ Eq. (A.13) yields $a(-Q)=a(Q)=-\left(f_{0}-f_{1}\right)$, so that $D(-Q ; \omega)$ has a degenerate zero at $\omega=0$, while the second pair of zeros is given by

$$
\omega_{s}^{2}(-Q)=-4 f_{1}\left(f_{0}-2 f_{1}\right)=-4 f_{1} \alpha
$$

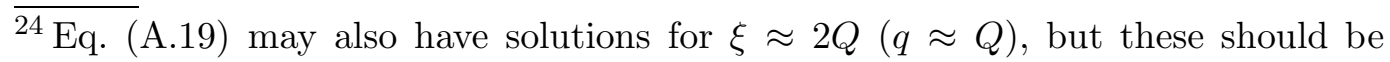
discarded since we have assumed $q \approx-Q$ in the perturbation calculation leading to Eq. (A.10). 
Thus, they are real only when $\alpha<0$, so that for small-amplitude SWs represented by elliptic periodic cycles there is always a non-oscillatory instability (with purely imaginary $\omega_{s}$ ) at wave vector $q=-Q$.

When $q=-Q+\xi$ with $\xi$ small, Eq. (A.8) becomes using again (A.13):

$$
a(\mp Q+\xi) \approx a(Q) \mp 2 \xi \sin Q=-\left(f_{1}+\alpha\right) \mp 2 \xi \sin Q
$$

which yields for the determinant (A.21) when also $f_{1}$ is small and $\alpha \ll f_{1}$ :

$$
D(-Q+\xi ; \omega) \approx \omega^{4}-4\left(2 \xi^{2} \sin ^{2} Q-f_{1} \alpha\right) \omega^{2}-48 \xi^{2} \alpha f_{1} \sin ^{2} Q
$$

Thus, at order $\xi^{2}$, Eq. (A.21) has no real zeros when

$$
f_{1} \alpha\left(f_{1} \alpha+8 \xi^{2} \sin ^{2} Q\right)<0
$$

For commensurate SWs represented by hyperbolic periodic cycles, $\alpha<0$. Then this condition becomes

$$
\xi^{2}>\frac{f_{1}}{8 \sin ^{2} Q}|\alpha|
$$

which proves that at small amplitude, the commensurate SWs represented by hyperbolic periodic cycles are unstable by oscillatory modulational instabilities with wave vectors $q$ close to but not equal to $\pm Q$.

Note that this proof does not hold for analytic incommensurate SWs because then $\alpha=0$ at order $f_{0}$. In that case, the first-order instabilities found in Sec. 4.2 for $0<Q<\pi / 2$ may occur outside the immediate neighborhood of $\omega=0$ where the condition $q \approx-Q$ assumed here is not valid.

\section{References}

[1] E. Fermi, J. Pasta, S. Ulam, Los Alamos Scientific Laboratory Report LA1940 (1955); also in: E. Segré (Ed.), Collected Papers of Enrico Fermi, vol. II, University of Chicago Press, Chicago, 1965, pp. 978-988.

[2] G. Iooss, K. Kirchgässner, Commun. Math. Phys. 211 (2000) 439-464.

[3] G. Iooss, Nonlinearity 13 (2000) 849-866.

[4] S. Aubry, Physica D 103 (1997) 201-250. 
[5] E. Lombardi, Oscillatory Integrals and Phenomena Beyond all Algebraic Orders, Lecture Notes in Mathematics. VOL. 1741, Springer, Berlin, 2000.

[6] S. Flach, C.R. Willis, Phys. Rep. 295 (1998) 181-264.

[7] T. Cretegny, S. Aubry, Phys. Rev. B 55 (1997) R11929-R11932.

[8] Yu.S. Kivshar, M. Peyrard, Phys. Rev. A 46 (1992) 3198-3205; I. Daumont, T. Dauxois, M. Peyrard, Nonlinearity 10 (1997) 617-630.

[9] T. Dauxois, S. Ruffo, A. Torcini, Phys. Rev. E 56 (1997) R6229-R6232.

[10] T. Ahn, R.S. MacKay, J.-A. Sepulchre, Dynamics of relative phases: generalised multibreathers, Nonlinear Dynamics, to appear.

[11] G. Kopidakis, S. Aubry, Physica D 130 (1999) 155-186.

[12] S. Aubry, in: R. McGehee, K.R. Meyer (Eds.), Twist Mappings and their Applications, Springer, New York, 1992, pp. 7-54; S. Aubry, G. Abramovici, Physica 43D (1990) 199-219.

[13] S. Aubry, in: A.R. Bishop, T. Schneider (Eds.), Solitons and Condensed Matter Physics, Springer, Berlin, 1978, pp. 264-278.

[14] S. Aubry, G. André, Ann. of the Israël Phys. Soc. 3 (1980) 133-164; reprinted in: P.J. Steinhardt, S. Ostlund (Eds.), The Physics of Quasicrystals, World Scientific, Singapore, 1987, pp. 554-593.

[15] S. Aubry, P.Y. Le Daëron, Physica 8D (1983) 381-422.

[16] Yu.S. Kivshar, M. Haelterman, A.P. Sheppard, Phys. Rev. E 50 (1994) 31613170.

[17] J.L. Marín, S. Aubry, Physica D 119 (1998) 163-174.

[18] M. Johansson, Yu.S. Kivshar, Phys. Rev. Lett. 82 (1999) 85-88.

[19] S. Aubry, J.-P. Gosso, G. Abramovici, J.-L. Raimbault, P. Quemerais, Physica D 47 (1991) 461-497.

[20] Ding Chen, S. Aubry, G.P. Tsironis, Phys. Rev. Lett. 77 (1996) 4776-4779.

[21] S. Aubry, T. Cretegny, Physica D 119 (1998) 34-46.

[22] Yu.S. Kivshar, W. Królikowski, O.A. Chubykalo, Phys. Rev. E 50 (1994) 50205032 .

[23] K.Ø. Rasmussen, T. Cretegny, P.G. Kevrekidis, N. Grønbech-Jensen, Phys. Rev. Lett. 84 (2000) 3740-3743.

[24] See, e.g., P. Bak, V.L. Pokrovsky, Phys. Rev. Lett. 47 (1981) 958-961; J. Coste, J. Peyraud, Phys. Rev. B 39 (1989) 13086-13095; Yi Wan, C.M. Soukoulis, Phys. Rev. A 41 (1990) 800-809; D. Hennig, G.P. Tsironis, Phys. Rep. 307, (1999) 333-432, and references therein. 
[25] A.M. Morgante, M. Johansson, G. Kopidakis, S. Aubry, Phys. Rev. Lett. 85 (2000) 550-553.

[26] S. Aubry, in: C. Godrèche (Ed.), Structures et Instabilités, les éditions de physique, Les Ulis, 1986, pp. 73-194 (in French).

[27] J.M. Greene, J. Math. Phys. 20 (1979) 1183-1201.

[28] J. Mather, Publ. Math. IHES 63 (1986) 153-204; A. Katok, B. Hasselblatt, Introduction to the Modern Theory of Dynamical Systems, Cambridge University Press, Cambridge, 1995, pp. 441-447.

[29] J.L. Marín, S. Aubry, Nonlinearity 9 (1996) 1501-1528.

[30] R.S. MacKay, S. Aubry, Nonlinearity 7 (1994) 1623-1643.

[31] J. Carr, J.C. Eilbeck, Phys. Lett. 109A (1985) 201-204; J.C. Eilbeck, P.S. Lomdahl, A.C. Scott, Physica 16D (1985) 318-338.

[32] V.I. Arnold, A. Avez, Ergodic problems of classical mechanics, Benjamin, New York, 1968, App. 29.

[33] M. Johansson, S. Aubry, Phys. Rev. E 61 (2000) 5864-5879.

[34] M. Johansson, S. Aubry, Nonlinearity 10 (1997) 1151-1178.

[35] A.M. Morgante, Ph.D. thesis, Ecole polytechnique, Palaiseau, France, 2001 (in French).

[36] S.F. Mingaleev, Yu.B. Gaididei, F.G. Mertens, Phys. Rev. E 61 (2000) R1044R1047; P.L. Christiansen, Y.B. Gaididei, F.G. Mertens, and S.F. Mingaleev, Eur. Phys. J. B 19 (2001) 545-553.

[37] J.L. Marín, S. Aubry, L.M. Floría, Physica D 113 (1998) 283-292.

[38] T. Cretegny, Ph.D. thesis, École normale supérieure de Lyon, France, 1998 (in French).

[39] M. Johansson (unpublished).

[40] H. Feddersen, in: M. Remoissenet, M. Peyrard (Eds.), Nonlinear Coherent Structures in Physics and Biology, Springer, Berlin, 1991, pp. 159-167.

[41] S.-S. Lee, S. Kim, Int. J. Mod. Phys. B 14 (2000) 1903-1914; S.W. Kim and S. Kim, Physica D 141 (2000) 91-103.

[42] V.V. Mirnov, A.J. Lichtenberg, H. Guclu, Physica D 157 (2001) 251-282.

[43] D.N. Christodoulides, R.I. Joseph, Opt. Lett. 13 (1988) 794-796; H.S. Eisenberg, Y. Silberberg, R. Morandotti, A.R. Boyd, J.S. Aitchison, Phys. Rev. Lett. 81 (1998) 3383-3386; R. Morandotti, U. Peschel, J.S. Aitchison, H.S. Eisenberg, Y. Silberberg, ibid. 83 (1999) 2726-2729.

[44] S. Aubry, R.S. MacKay, C. Baesens, Physica D 56 (1992) 123-134. 